\title{
Ultraviolet behavior of $6 D$ supersymmetric Yang-Mills theories and harmonic superspace
}

\author{
Guillaume Bossard, ${ }^{a}{\text { Evgeny } \text { Ivanov }^{b} \text { and Andrei Smilga }}^{c}$ \\ ${ }^{a}$ Centre de Physique Théorique, Ecole Polytechnique, CNRS, Université Paris-Saclay, \\ 91128 Palaiseau cedex, France \\ ${ }^{b}$ Bogoliubov Laboratory of Theoretical Physics, JINR, \\ 6 Joliot-Curie str., 141980 Dubna, Moscow region, Russia \\ ${ }^{c} S U B A T E C H$, Université de Nantes, \\ 4 rue Alfred Kastler, BP 20722, Nantes 44307, France \\ E-mail: bossard@cpht.polytechnique.fr, eivanov@theor.jinr.ru, \\ smilga@subatech.in2p3.fr
}

ABSTRACT: We revisit the issue of higher-dimensional counterterms for the $\mathcal{N}=(1,1)$ supersymmetric Yang-Mills (SYM) theory in six dimensions using the off-shell $\mathcal{N}=(1,0)$ and on-shell $\mathcal{N}=(1,1)$ harmonic superspace approaches. The second approach is developed in full generality and used to solve, for the first time, the $\mathcal{N}=(1,1) \mathrm{SYM}$ constraints in terms of $\mathcal{N}=(1,0)$ superfields. This provides a convenient tool to write explicit expressions for the candidate counterterms and other $\mathcal{N}=(1,1)$ invariants and may be conducive to proving non-renormalization theorems needed to explain the absence of certain logarithmic divergences in higher-loop contributions to scattering amplitudes in $\mathcal{N}=(1,1)$ SYM.

Keywords: Supersymmetric gauge theory, Extended Supersymmetry, Field Theories in Higher Dimensions, Supersymmetric Effective Theories

ArXiv EPRINT: 1509.08027

Dedicated to the memory of Boris Zupnik 


\section{Contents}

1 Introduction 1

2 Off-shell vs. on-shell 5

2.1 Witten's model 5

$\begin{array}{lll}2.2 & 4 D \text { supersymmetric gauge theories } & 7\end{array}$

3 Harmonic superspace and harmonic superfields $\quad 8$

$\begin{array}{llr}3.1 & \text { Spinor algebra } & 8\end{array}$

$\begin{array}{ll}3.2 \text { Superspace } & 10\end{array}$

$\begin{array}{lll}3.3 & \text { Superfields } & 11\end{array}$

4 Invariant actions of the $\mathcal{N}=(1,0)$ vector multiplet and a hypermultiplet 14

4.1 The dimension 4 Lagrangian of the gauge multiplet 14

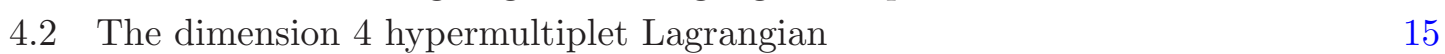

4.3 The $\mathcal{N}=(1,1)$ SYM action and its hidden $\mathcal{N}=(0,1)$ supersymmetry $\quad 15$

5 Higher-dimensional $\mathcal{N}=(1,0)$ and $\mathcal{N}=(1,1)$ invariants 18

$\begin{array}{lll}5.1 & d=6 & 18\end{array}$

$\begin{array}{lll}5.2 d=8 & 20\end{array}$

5.3 On-shell $\mathcal{N}=(1,0)$ and $\mathcal{N}=(1,1)$ invariants $\quad 22$

6 On-shell $\mathcal{N}=(1,1)$ harmonic superfields $\quad 25$

6.1 The standard and harmonic $\mathcal{N}=(1,1)$ superspaces 25

$\begin{array}{lll}6.2 & \text { From the central basis to the analytic basis } & 27\end{array}$

7 Solving the $\mathcal{N}=(1,1)$ SYM constraints in terms of $\mathcal{N}=(1,0)$ super-

fields $\quad 30$

$\begin{array}{lll}7.1 & \text { Input and gauge-fixing } & 30\end{array}$

$\begin{array}{lll}7.2 & \text { Harmonic equations } & 32\end{array}$

$\begin{array}{lll}7.3 & \text { Identifying vector connections } & 36\end{array}$

8 On-shell $\mathcal{N}=(1,1)$ supersymmetric actions $\quad 39$

$\begin{array}{ll}8.1 \text { Invariant actions: } d=8 & 39\end{array}$

8.1.1 Gauge non-invariant off-shell supersymmetric realization $\quad 41$

$\begin{array}{ll}8.2 \text { Invariant actions: } d=10 & 42\end{array}$

9 Summary and outlook $\quad 46$

$\begin{array}{ll}\text { A Bianchi identities } & \mathbf{4 8}\end{array}$

$\begin{array}{lll}\text { A.1 Off-shell relations } & 48\end{array}$

A.2 On-shell $\mathcal{N}=(1,1)$ relations $\quad 49$ 
C.2 Hidden $\mathcal{N}=(0,1)$ supersymmetry 53

\section{Introduction}

Yang-Mills theory and its supersymmetric extensions have been studied extensively over the years, and are of particular relevance in four dimensions, in which case they define renormalizable quantum field theories. It is well known that these theories are not renormalizable by power counting in higher dimensions, but they nonetheless provide effective theory descriptions of some particular low energy sectors of string theory, such as D5brane dynamics and open string theory compactifications. In this paper, we concentrate on $6 D$ supersymmetric Yang-Mills (SYM) theory. Only the maximally supersymmetric $\mathcal{N}=(1,1)$ theory, involving both left-handed and right-handed supercharges, is anomaly free in six dimensions [1-4], and is therefore physically relevant. The effective action for coincident D5-branes defines a non-abelian generalization of Born-Infeld theory representing an infinite series that involves the standard $\mathcal{N}=(1,1)$ supersymmetric $6 D$ Yang-Mills Lagrangian and higher-derivative corrections [5-9].

We wish to note right away that the individual terms in the effective action need not and do not possess the full extended supersymmetry that string theory enjoys. Only the whole infinite series has this property. We will discuss this issue in detail later.

The higher-derivative supersymmetric structures similar to those that appear in the Born-Infeld action define also the candidate counterterms for the ultra-violet (UV) logarithmic divergences in the $6 D$ SYM theory. The supersymmetric Ward identities for the on-shell amplitudes only require these counterterms (at least, the counterterms that are responsible for first logarithmic divergences $\sim \ln \Lambda_{U V}$ in the amplitudes) to be invariant under extended supersymmetry transformations on mass shell, i.e. modulo the equations of motion of the $6 D$ SYM theory. Only when one can give a superspace formulation of the theory and use a symmetry-preserving regularization, the counterterms should possess the corresponding supersymmetry off shell.

There is no off-shell $\mathcal{N}=(1,1)$ superspace formulation of the maximally supersymmetric $6 D$ SYM theory. Thus, we cannot expect the counterterms to enjoy the full off-shell supersymmetry of the original action. The on-shell $\mathcal{N}=(1,1)$ supersymmetry should, however, be there. On the other hand, there exists a $\mathcal{N}=(1,0)$ superspace formulation, and the relevant counterterms should be $\mathcal{N}=(1,0)$ off-shell [and $\mathcal{N}=(1,1)$ on-shell] supersymmetric. A limited symmetry of relevant counterterms is a specific feature of theories with extended supersymmetry. In more simple cases (think of the Euler-Heisenberg effective Lagrangian for QED or of higher-dimensional counterterms in the effective chiral theory describing the low-energy sector of QCD), all individual terms in the effective Lagrangian possess the same off-shell symmetries as the leading term. 
The structure of higher-dimensional counterterms was previously studied in the conventional superspace approach [10] and in (on-shell) harmonic superspace in [11]. A convenient way to determine the structure of these counterterms is using the (off-shell) harmonic superspace technique developed in $[12,13]$ and extended to six dimensions in [14-16]. That is what we do in the present paper.

We make use of the $\mathcal{N}=(1,0)$ off-shell harmonic superspace formalism of refs. [14-16], to define in detail the $\mathcal{N}=(1,1)$ on-shell harmonic superspace invariants introduced in [11]. We rewrite the standard superspace $\mathcal{N}=(1,1)$ SYM constraints in $\mathcal{N}=(1,1)$ harmonic superspace. The main new result here is to solve explicitly these constraints in terms of $\mathcal{N}=$ $(1,0)$ superfields. Since the constraints put the theory on shell, the $\mathcal{N}=(1,0)$ superfields are necessarily subjected to their equations of motion. Nevertheless, while constructing the invariants from the constrained $\mathcal{N}=(1,1)$ superfield strength as integrals over superspaces involving the full $\mathcal{N}=(1,0)$ superspace as a subspace, these superfields can still be treated as off-shell ones. The on-shell conditions are needed only while checking the hidden $\mathcal{N}=$ $(0,1)$ supersymmetry of these invariants. The $\mathcal{N}=(1,1)$ harmonic superspace formalism allowed us to write down explicit analytic expressions in off-shell $\mathcal{N}=(1,0)$ harmonic superspace for the candidate counterterms. Their analysis may help to understand the reason why certain possible a priori logarithmically divergent structures in the scattering amplitudes happen to be absent, as was displayed in explicit 3-loop calculations [17-19]. ${ }^{1}$

The algebraic renormalization method [20] was generalized to non-renormalizable supersymmetric theories in [11] as a tool to determine whether some counterterms could support logarithmic divergences. This allowed to explain the absence of 2-loop divergences. But the same arguments fail to explain the absence of non-planar divergences at the 3-loop level [21]. Arguments using the pure spinor formalism [22-24] allow to explain this result, but there is no direct quantum field theory understanding of this non-renormalization theorem. We will see that the absence of 2-loop divergences can also be understood in the $\mathcal{N}=(1,0)$ harmonic superspace framework through the absence of an $\mathcal{N}=(1,0)$ off-shell supersymmetric and manifestly gauge invariant counterterm of canonical dimension $d=8 .^{2}$ At the 3-loop level $(d=10)$, both planar (or single-trace) and non-planar (or double-trace) supersymmetric counterterms can be constructed. We shall argue, however, that Ward identities combining the algebraic approach for non-linear hidden supersymmetry with the off-shell $\mathcal{N}=(1,0)$ harmonic superspace methods could potentially explain the 3 -loop non-renormalization theorem.

The $6 D$ SYM theory also represents an interest as a toy model for more complicated extended supergravity theories. In particular, the absence of divergences for the doubletrace structure in the 3-loop amplitude in six dimensions obtained by explicit computations is somehow similar to the absence of divergences which was observed for the four-graviton amplitude in $\mathcal{N}=4$ supergravity in four dimensions at the 3-loop level and in $\mathcal{N}=5$ supergravity at the 4-loop level [25-28].

\footnotetext{
${ }^{1}$ We are talking here only about logarithmic divergences; power UV divergences characteristic of a nonrenormalizable theory are present starting from the first loop in certain UV regularization schemes Slavnov's higher-derivative scheme or lattice regularization. These power divergences cannot be cared of by calculations in the papers just cited.

${ }^{2}$ Hereafter, we denote by $d$ the canonical dimension (in mass units) of the relevant component $6 D$ Lagrangian.
} 
Indeed, for pure $\mathcal{N}=4$ supergravity (without matter), the first available supersymmetric counterterm appears at the 3-loop level [29, 30]. The absence of anomaly for the Cremmer-Julia symmetry for $\mathcal{N}$-extended supergravity with $\mathcal{N} \geq 5$ [31] and inspection of the possible supersymmetry invariants exhibit that the first available counterterm only appears at $(\mathcal{N}-1)$-loop order [32-34]. This allows to understand the good ultra-violet behavior of $\mathcal{N}=8$ supergravity amplitudes which was observed in [35-37] through four loops. However, this symmetry principle fails to explain the absence of divergences at three loops in pure $\mathcal{N}=4$ supergravity [38], as well as at four loops in $\mathcal{N}=5$ supergravity. These unexplained cancelations suggest that, by the same currently unexplained reason, maximal supergravity may not diverge at seven loops, in spite of the presence of a counterterm satisfying all symmetries [34]. On the other hand, the 4-loop amplitudes in $\mathcal{N}=4$ supergravity are known to involve logarithmic divergences, and one might think that the same is true for the 8-loop amplitudes in the maximal $\mathcal{N}=8$ supergravity, as was predicted long time ago in [39].

Although the non-renormalization theorems in $6 D$ SYM theory and in supergravity were proven using different methods, one may hope that a future proof of the nonrenormalization theorem for the non-planar 3-loop logarithm divergence in Yang-Mills theory could shed some light on possible generalizations to supergravity.

The structure of the paper is the following.

In section 2, we attempt to give a pedagogical explanation of the above-mentioned fact that the individual terms in the supersymmetric effective Lagrangian do not necessarily possess all the symmetries of the leading term. We illustrate this for the toy supersymmetric quantum mechanical model with only one bosonic degree of freedom.

In section 3 , we recall the basic notions and introduce notation for 6-dimensional $\mathcal{N}=$ $(1,0)$ harmonic superspace. In section 4 , we use this formalism to construct the classical invariant actions of canonical dimension 4 involving the $\mathcal{N}=(1,0)$ vector multiplet and the hypermultiplet. One of such actions enjoys the extended $\mathcal{N}=(1,1)$ supersymmetry, with the $\mathcal{N}=(0,1)$ part of this symmetry being realized via the transformations of $\mathcal{N}=(1,0)$ harmonic superfields.

In section 5 , still working in the $\mathcal{N}=(1,0)$ superspace framework, we analyze higherdimensional $\mathcal{N}=(1,1)$ supersymmetric Lagrangians. We show that

- At the 1-loop level $(d=6)$, all the candidate counterterms vanish on mass shell [40, 41]. We demonstrate in section 6.1 and, in more details, in appendix B that no $d=6$ off-shell $\mathcal{N}=(1,1)$ supersymmetric Lagrangian can be constructed.

- At the 2-loop level $(d=8)$, the candidate counterterms also vanish on mass shell, if we require them to be $\mathcal{N}=(1,0)$ off-shell supersymmetric and gauge invariant. ${ }^{3}$

\footnotetext{
${ }^{3}$ These requirements should be imposed under the assumption that the perturbative calculations can be done in a way that preserves at all steps the off-shell $\mathcal{N}=(1,0)$ supersymmetry and gauge invariance, both of them being kept by regularization. This assumption is very natural: the existence of $\mathcal{N}=(1,0)$ superspace implies the existence of supergraph technique, and the higher-derivative ultraviolet regularization keeps gauge invariance and $\mathcal{N}=(1,0)$ supersymmetry, but we are not aware of its formal rigorous proof.
} 
- On the other hand, one can construct an on-shell $d=8$ gauge-invariant Lagrangian involving both the vector multiplet and hypermultiplet and possessing both $\mathcal{N}=$ $(1,0)$ and $\mathcal{N}=(0,1)$ supersymmetries only on shell. Its bosonic part starts with the structure $\sim F^{4}$. It does not appear as a counterterm for the $\mathcal{N}=(1,1), 6 D$ SYM theory, but is present in the derivative expansion of the Born-Infeld action for coincident D5-branes.

The methods of section 5 where the extra $\mathcal{N}=(0,1)$ supersymmetry is "hidden" in the superfield transformations proved not to be too efficient for constructing the 3-loop $d=10$ invariants; even the construction of the $d=8$ invariants by this "brute force" method is rather complicated technically. To perform such a study in a more systematic way, we developed in section 6 and 7 the on-shell $\mathcal{N}=(1,1)$ harmonic superfield formalism. It involves a double set of harmonics, $u_{i}^{ \pm}$and $u_{A}^{\hat{ \pm}}$, as well as the extra $\mathrm{SU}(2)$ doublet of the $(0,1)$ chiral $6 D$ fermionic superspace coordinates. We show that

- The known superspace constraints on the covariant spinor derivatives, which define the $\mathcal{N}=(1,1)$ SYM theory $[10,42]$, admit a compact rewriting in this bi-harmonic superspace as the conditions for the two types of covariant Grassmann harmonic analyticities.

- These constraints are explicitly solved in section 7 in terms of the $\mathcal{N}=(1,0) \mathrm{SYM}$ gauge superfield and hypermultiplet, simultaneously providing the $\mathcal{N}=(1,0)$ Grassmann harmonic analyticity and the on-shell conditions for these superfields.

- These $\mathcal{N}=(1,0)$ constituents are encompassed by the single double-analytic $\mathcal{N}=$ $(1,1)$ superfield strength with simple transformation properties under the $\mathcal{N}=(0,1)$ supersymmetry.

In section 8 , we write various invariant actions in terms of this $\mathcal{N}=(1,1)$ superfield strength as integrals over the full $\mathcal{N}=(1,1)$ superspace or its $1 / 2$ or $3 / 4$ analytic subspaces and further rewrite these invariants in the $\mathcal{N}=(1,0)$ superspaces.

- We rederive in this way the on-shell $d=8$ invariant obtained in section 5 and also derive nontrivial expressions for the single-trace and double-trace $d=10$ invariants in terms of $\mathcal{N}=(1,0)$ superfields. We note that the single-trace invariant can be represented as a full $\mathcal{N}=(1,1)$ superspace integral, whereas the double-trace invariant cannot. We suggest that, using the algebraic method in $\mathcal{N}=(1,0)$ harmonic superspace, this may be enough for proving a non-renormalization theorem preventing the appearance of the double trace as a counterterm.

- We also present an alternative view of constructing higher-order invariants on the $d=8$ example. One can keep the off-shell $\mathcal{N}=(1,0)$ supersymmetry, but allow for the gauge invariance to be deformed, or modify the definition of the Yang-Mills field strength curvature [43]. We write the explicit expression for the $d=8$ action thus obtained. This may provide an alternative way to construct the supersymmetric Born-Infeld Lagrangian in $\mathcal{N}=(1,0)$ harmonic superspace, although we do not investigate this issue in this paper. 
There are three technical appendices. In appendix A, we derive certain Bianchi identities relating different $\mathcal{N}=(1,0)$ superfields that are used in section 5 . In appendix $\mathrm{B}$, we describe a failed attempt to construct an off-shell $\mathcal{N}=(1,1)$ invariant $d=6$ action. We conclude that such an action in all probability does not exist. In appendix $\mathrm{C}$, we give an alternative derivation of the $d=8$ on-shell $\mathcal{N}=(1,1)$ supersymmetric Lagrangian, directly in the $\mathcal{N}=(1,0)$ superspace.

\section{Off-shell vs. on-shell}

In this pedagogical section, we clarify generic features of effective supersymmetric Lagrangians by studying in detail two toy supersymmetric quantum mechanical models and recalling the familiar situation for $4 D$ field theories.

\section{$2.1 \quad$ Witten's model}

The simplest possible example is Witten's supersymmetric quantum mechanical system involving one bosonic degree of freedom with the Lagrangian [44]

$$
L_{0}=\frac{\dot{x}^{2}-\left[V^{\prime}(x)\right]^{2}}{2}+\frac{i}{2}(\dot{\psi} \bar{\psi}-\psi \dot{\bar{\psi}})+V^{\prime \prime}(x) \bar{\psi} \psi
$$

The corresponding equations of motion are

$$
\begin{aligned}
\ddot{x}+V^{\prime}(x) V^{\prime \prime}(x)-V^{\prime \prime \prime}(x) \bar{\psi} \psi & =0, \\
i \dot{\psi}-V^{\prime \prime}(x) \psi & =0, \\
i \dot{\bar{\psi}}+V^{\prime \prime}(x) \bar{\psi} & =0 .
\end{aligned}
$$

The Lagrangian (2.1) is invariant (up to a total time derivative) under the following nonlinear supersymmetry transformations

$$
\begin{aligned}
\delta x & \equiv \delta_{\epsilon} x+\delta_{\bar{\epsilon}} x=\epsilon \bar{\psi}+\psi \bar{\epsilon}, \\
\delta \psi & \equiv \delta_{\epsilon} \psi=-\epsilon\left[i \dot{x}+V^{\prime}(x)\right], \\
\delta \bar{\psi} & \equiv \delta_{\bar{\epsilon}} \bar{\psi}=\bar{\epsilon}\left[i \dot{x}-V^{\prime}(x)\right] .
\end{aligned}
$$

Note now that it is impossible to write a Lagrangian depending on the fields $x, \psi, \bar{\psi}$ and involving their higher time derivatives which would be invariant under the transformations (2.3). This is due to the well-known fact that the Lie brackets of the transformations (2.3) do not close off mass shell, but only on mass shell. When acting on the variable $x(t)$, the Lie bracket $\left(\delta_{\bar{\epsilon}} \delta_{\xi}-\delta_{\xi} \delta_{\bar{\epsilon}}\right)$ boils down to a total time derivative. But it is not so for the fermion variables. For example,

$$
\left(\delta_{\bar{\epsilon}} \delta_{\xi}-\delta_{\xi} \delta_{\bar{\epsilon}}\right) \psi=\xi \bar{\epsilon}\left[i \dot{\psi}+V^{\prime \prime}(x) \psi\right]=2 i \xi \bar{\epsilon} \dot{\psi}+\xi \bar{\epsilon} \frac{\partial L}{\partial \bar{\psi}} .
$$

The presence of the second term in (2.4) does not affect the invariance of $L_{0}$ under (2.3). Indeed,

$$
\left(\delta_{\bar{\epsilon}} \delta_{\xi}-\delta_{\xi} \delta_{\bar{\epsilon}}\right) L_{0}=2 i \xi \bar{\epsilon} \dot{L_{0}}+\xi \bar{\epsilon}\left(\frac{\partial L_{0}}{\partial \psi} \frac{\partial L_{0}}{\partial \bar{\psi}}+\frac{\partial L_{0}}{\partial \bar{\psi}} \frac{\partial L_{0}}{\partial \psi}\right)=2 i \xi \bar{\epsilon} \dot{L_{0}}
$$

But, for $L \neq L_{0}$, the second term in the Lie bracket $\left(\delta_{\bar{\epsilon}} \delta_{\xi}-\delta_{\xi} \delta_{\bar{\epsilon}}\right) L$ vanishes only on the mass shell of $L_{0}$. 
The standard way to solve this problem and to construct fully supersymmetric actions of any dimension is to introduce a superfield

$$
X(t, \theta, \bar{\theta})=x+\theta \bar{\psi}+\psi \bar{\theta}+F \theta \bar{\theta}
$$

The transformations of the superspace coordinates generate linear supersymmetry transformations of the dynamic variables,

$$
\begin{aligned}
\delta x & =\epsilon \bar{\psi}+\psi \bar{\epsilon}, \\
\delta \psi & =\epsilon(F-i \dot{x}), \\
\delta \bar{\psi} & =\bar{\epsilon}(F+i \dot{x}), \\
\delta F & =i(\epsilon \dot{\bar{\psi}}-\dot{\psi} \bar{\epsilon}) .
\end{aligned}
$$

Any higher-derivative action of the form

$$
S=\int d t d \bar{\theta} d \theta\left(\frac{1}{2} \bar{D} X P\left[\frac{\partial}{\partial t}\right] D X+V(X)\right)
$$

where $P(\partial / \partial t)$ is an arbitrary polynomial and

$$
D=\frac{\partial}{\partial \theta}+i \bar{\theta} \frac{\partial}{\partial t}, \quad \bar{D}=-\frac{\partial}{\partial \bar{\theta}}-i \theta \frac{\partial}{\partial t}
$$

are the supersymmetric covariant derivatives, is invariant under (2.7).

For a linear polynomial $P(z)=a+b z$, one obtains an interesting higher-derivative model whose Hamiltonian is Hermitian in spite of the presence of the ghosts (no ground state in the spectrum) [45]. For higher-order polynomials, the Hermiticity is lost, but we need not worry about it, we use this as a toy model displaying the structure of the effective Wilsonian Lagrangian in a field theory of interest. We choose $P(z)=1-g z^{2}$. One obtains then the following component Lagrangian,

$$
L=\frac{1}{2}\left(\dot{x}^{2}+F^{2}\right)+i \dot{\bar{\psi}} \psi+F V^{\prime}(x)+V^{\prime \prime}(x) \bar{\psi} \psi+g \frac{1}{2}\left(\ddot{x}^{2}+\dot{F}^{2}+2 i \ddot{\bar{\psi}} \dot{\psi}\right) .
$$

This Lagrangian is invariant under the transformations (2.7). On the other hand, the formerly auxiliary field $F$ has become dynamical and cannot be algebraically eliminated as it can in Witten's model with $g=0$. Still, one can integrate out the field $F$ perturbatively through the formal power series solution

$$
F=-\sum_{n=0}^{\infty} g^{n} \frac{d^{2 n} V^{\prime}(x)}{d t^{2 n}}
$$

One obtains in this way the Lagrangian

$$
L=\frac{1}{2}\left(\dot{x}^{2}+g \ddot{x}^{2}\right)+i \dot{\bar{\psi}} \psi+i g \ddot{\bar{\psi}} \dot{\psi}-\frac{1}{2} \sum_{n=0}^{\infty}(-g)^{n}\left(\frac{d^{n} V^{\prime}(x)}{d t^{n}}\right)^{2}+V^{\prime \prime}(x) \bar{\psi} \psi,
$$


which is by construction invariant with respect to the nonlinear supersymmetry transformations

$$
\begin{aligned}
& \delta x=\epsilon \bar{\psi}+\psi \bar{\epsilon} \\
& \delta \psi=\epsilon\left(-i \dot{x}-\sum_{n=0}^{\infty} g^{n} \frac{d^{2 n} V^{\prime}(x)}{d t^{2 n}}\right), \quad \delta \bar{\psi}=\bar{\epsilon}\left(i \dot{x}-\sum_{n=0}^{\infty} g^{n} \frac{d^{2 n} V^{\prime}(x)}{d t^{2 n}}\right),
\end{aligned}
$$

that close modulo the equations of motion for the full Lagrangian (2.12). For example,

$$
\left(\delta_{\epsilon_{1}} \delta_{\epsilon_{2}}-\delta_{\epsilon_{2}} \delta_{\epsilon_{1}}\right) \psi=-2 \epsilon_{1} \epsilon_{2} \sum_{n=0}^{\infty} g^{n} \frac{d^{2 n}}{d t^{2 n}}\left(\frac{\partial L}{\partial \psi}\right) .
$$

The Lagrangian (2.12) represents a perturbative series in $g$,

$$
L=\sum_{n=0}^{\infty} g^{n} L_{n}=L_{0}+g L_{1}+g^{2} L_{2}+\ldots
$$

and similarly for $\delta=\delta_{0}+g \delta_{1}+\ldots$, where $L_{0}$ is written in (2.1) and $\delta_{0}$ in (2.3). It follows by construction that the first-order correction,

$$
L_{1}=\frac{1}{2} \ddot{x}^{2}+i \ddot{\bar{\psi}} \dot{\psi}+\frac{1}{2} \dot{x}^{2}\left(V^{\prime \prime}(x)\right)^{2},
$$

is invariant under the action of $\delta_{0}$ modulo the classical equations of motion (2.2) and a total time derivative,

$$
\delta_{0} L_{1}+\delta_{1} L_{0}=\frac{d}{d t}(\cdots) .
$$

In other words, the action $\int d t L_{1}$ is invariant with respect to nonlinear supersymmetry transformations (2.3) on shell, but not off shell.

On the contrary, the second-order correction

$$
L_{2}=-\frac{1}{2}\left(\ddot{x} V^{\prime \prime}(x)+\dot{x}^{2} V^{\prime \prime \prime}(x)\right)^{2},
$$

is not invariant with respect to $\delta_{0}$, but satisfies instead

$$
\delta_{0} L_{2}+\delta_{1} L_{1}+\delta_{2} L_{0}=\frac{d}{d t}(\cdots)
$$

The situation when the effective Lagrangian represents an infinite series of higherderivative terms, like in (2.12), and this Lagrangian is invariant under modified supersymmetry transformations also representing an infinite series, is quite general. One known example is the Born-Infeld effective Lagrangian mentioned in the introduction.

\section{$2.24 D$ supersymmetric gauge theories}

Consider first the $\mathcal{N}=1,4 D$ supersymmetric SYM Lagrangian. It involves the gauge fields and gluinos and is invariant under certain nonlinear supersymmetry transformations. One also can write higher-derivative off-shell supersymmetric Lagrangians of canonical 
dimensions $d=6,8$, etc., but they necessarily include the auxiliary field $D$ of the vector multiplet, which now becomes dynamical. In this case, supersymmetry is realized linearly.

The same is true for the $\mathcal{N}=2$ supersymmetric SYM theory. We have a scalar superfield $\mathcal{W}$ involving a triplet of auxiliary fields $D^{A}$. Higher-derivatives supersymmetric Lagrangians, like $\mathcal{L} \sim \operatorname{Tr} \int d^{8} \theta \mathcal{W}^{2} \overline{\mathcal{W}}^{2}$, can be written, and they involve the derivatives of $D^{A}$. For the "matter" fields belonging to the $\mathcal{N}=2$ hypermultiplet, the full set of the auxiliary fields is infinite. The latter can be presented as components of a certain $\mathcal{N}=2$ harmonic superfield. Higher-derivative off-shell invariant actions can also be written in that case.

But for the $\mathcal{N}=4$ theory, the situation is different. Superfield formalism, with all supersymmetries being manifest and off-shell, is not developed, the full set of auxiliary fields is not known and probably does not exist. Thus, one cannot write in this case an off-shell supersymmetric higher-derivative action. On the other hand, nontrivial higherderivative actions enjoying on-shell $\mathcal{N}=4$ supersymmetry exist (see, e.g., [9, 46-48]).

In four dimensions, these higher-derivative invariants are not relevant for perturbative calculations - they do not appear as counterterms for a renormalizable (even finite for $\mathcal{N}=4$ ) theory. ${ }^{4}$ But such invariants are relevant in six dimensions. As far as their structure is concerned, the situation is the same as in four dimensions. Using harmonic approach, one can develop $\mathcal{N}=(1,0)$ harmonic superfield formalism and write down many off-shell $\mathcal{N}=(1,0)$ invariants. On the other hand, we have no off-shell $\mathcal{N}=(1,1)$ superfield formalism and off-shell $\mathcal{N}=(1,1)$ invariants probably do not exist. However, it is possible to write down many on-shell $\mathcal{N}=(1,1)$ invariants, and we will do it explicitly for the canonical dimensions $d=8$ and $d=10$.

\section{Harmonic superspace and harmonic superfields}

We give here some basic facts about the $6 D$ spinor algebra, the ordinary and harmonic $\mathcal{N}=(1,0)$ superspaces and $\mathcal{N}=(1,0)$ superfields. For more details, see refs. [40, 41].

\subsection{Spinor algebra}

The group $\operatorname{Spin}(5,1)$ possesses two different spinor representations, the complex 4component spinors $\lambda^{a}$ and the complex 4-component spinors $\psi_{a}$. In contrast to $\operatorname{Spin}(6) \equiv$ $\mathrm{SU}(4)$, where two 4-dimensional representations are related to each other by complex conjugation, in Spin $(5,1)$, they are completely independent. The situation is opposite to that in $4 D$ where the group $\operatorname{Spin}(3,1)$ involves two complex conjugate spinor representations, while in $\operatorname{Spin}(4) \equiv \mathrm{SU}(2) \times \mathrm{SU}(2)$ these representations are independent.

For the vectors, it is convenient to introduce the notation

$$
V_{a b}=\frac{1}{2}\left(\gamma^{M}\right)_{a b} V_{M}
$$

\footnotetext{
${ }^{4}$ Though they can appear in the Wilsonian effective actions.
} 
where $M=0, \ldots, 5$ and $\left(\gamma^{M}\right)_{a b}$ are antisymmetric $6 D$ matrices (the $6 D$ analog of $\sigma_{\mu}$ ) satisfying

$$
\gamma_{M} \tilde{\gamma}_{N}+\gamma_{N} \tilde{\gamma}_{M}=-2 \eta_{M N}, \quad \eta_{M N}=\operatorname{diag}(1,-1,-1,-1,-1,-1),
$$

with

$$
\left(\tilde{\gamma}_{M}\right)^{a b}=\frac{1}{2} \varepsilon^{a b c d}\left(\gamma_{M}\right)_{c d} .
$$

One of the possible explicit representations of these matrices is

$$
\begin{array}{lll}
\gamma_{0}=\tilde{\gamma}_{0}=i \sigma_{2} \otimes \mathbb{1} ; & \gamma_{1}=-\tilde{\gamma}_{1}=i \sigma_{2} \otimes \sigma_{1} ; & \gamma_{2}=-\tilde{\gamma}_{2}=i \mathbb{1} \otimes \sigma_{2} ; \\
\gamma_{3}=-\tilde{\gamma}_{3}=i \sigma_{2} \otimes \sigma_{3} ; & \gamma_{4}=\tilde{\gamma}_{4}=\sigma_{1} \otimes \sigma_{2} ; & \gamma_{5}=\tilde{\gamma}_{5}=\sigma_{3} \otimes \sigma_{2} .
\end{array}
$$

Note the properties

$$
\operatorname{Tr}\left\{\gamma_{M} \tilde{\gamma}_{N} \gamma_{P} \tilde{\gamma}_{Q} \gamma_{R} \tilde{\gamma}_{S}\right\}=-\operatorname{Tr}\left\{\tilde{\gamma}_{M} \gamma_{N} \tilde{\gamma}_{P} \gamma_{Q} \tilde{\gamma}_{R} \gamma_{S}\right\}=4 \varepsilon_{M N P Q R S}+\text { symmetric part }
$$

$\left(\varepsilon_{012345}=1\right)$ and

$$
\left(\gamma^{A}\right)_{a b}\left(\gamma_{A}\right)_{c d}=2 \varepsilon_{a b c d}, \quad\left(\tilde{\gamma}^{A}\right)^{a b}\left(\tilde{\gamma}_{A}\right)^{c d}=2 \varepsilon^{a b c d}
$$

The Dirac gamma-matrices $\Gamma_{M}$ satisfying the standard Clifford algebra

$$
\Gamma_{M} \Gamma_{N}+\Gamma_{N} \Gamma_{M}=2 \eta_{M N}
$$

can be chosen as

$$
\Gamma_{M}=\left(\begin{array}{cc}
0 & \tilde{\gamma}_{M} \\
-\gamma_{M} & 0
\end{array}\right)
$$

One can also introduce

$$
\Gamma_{7}=\Gamma_{0} \Gamma_{1} \Gamma_{2} \Gamma_{3} \Gamma_{4} \Gamma_{5}
$$

and observe that the spinors $\lambda^{a}, \psi_{a}$ are the chiral left-handed and right-handed projections of a 8-component Dirac spinor, i.e. $\lambda, \psi=\left(1 \pm \Gamma_{7}\right) \Psi / 2$.

The $\operatorname{Spin}(5,1)$ generators are

$$
\left(\sigma_{M N}\right)^{a}{ }_{b}=\frac{1}{2}\left(\tilde{\gamma}_{M} \gamma_{N}-\tilde{\gamma}_{N} \gamma_{M}\right)^{a}{ }_{b}=\frac{1}{2}\left(\gamma_{N} \tilde{\gamma}_{M}-\gamma_{M} \tilde{\gamma}_{N}\right)_{b}{ }^{a}
$$

They are real. ${ }^{5}$ This makes it convenient to define, instead of a complex 4-component spinor $\lambda^{a}$, a couple of spinors $\lambda_{j=1,2}^{a}$ obeying the pseudoreality condition

$$
\overline{\lambda_{j}^{a}} \equiv-C_{b}^{a}\left(\lambda_{j}^{b}\right)^{*}=\varepsilon^{j k} \lambda_{k}^{a},
$$

where $C$ is the charge conjugation matrix with the properties $C=-C^{T}, C^{2}=-1$. It can be chosen as $C=\tilde{\gamma}_{0} \gamma_{5}$.

Similarly, instead of a generic complex $\psi_{a}$, one can introduce a couple of right-handed spinors $\psi_{a}^{A}$ related by the pseudoreality condition.

\footnotetext{
${ }^{5}$ Thus, the algebra $\operatorname{spin}(5,1)$ represents a real form of $\operatorname{spin}(6) \equiv s u(4)$ and is sometimes denoted $s u^{*}(4)$.
} 


\subsection{Superspace}

The standard $\mathcal{N}=(1,0)$ superspace involves the coordinates

$$
z=\left(x^{M}, \theta_{i}^{a}\right)
$$

where $\theta_{i}^{a}$ are Grassmann pseudoreal left-handed spinors.

Next we introduce the harmonics $u^{ \pm i}\left(u_{i}^{-}=\left(u_{i}^{+}\right)^{*}, u^{+i} u_{i}^{-}=1\right)$, which describe the "harmonic sphere" $\mathrm{SU}(2)_{R} / \mathrm{U}(1)$, where $\mathrm{SU}(2)_{R}$ is R-symmetry group of the $\mathcal{N}=(1,0)$ Poincaré superalgebra. ${ }^{6}$ The harmonic $\mathcal{N}=(1,0), 6 D$ superspace in the central basis is parametrized by the coordinates

$$
Z:=(z, u)=\left(x^{M}, \theta_{i}^{a}, u^{ \pm i}\right)
$$

The harmonic superspace in the analytic basis involves the harmonics and the coordinates $x_{(\mathrm{an})}^{M}, \theta^{ \pm a}$ :

$$
\begin{aligned}
Z_{(\mathrm{an})} & :=\left(x_{(\mathrm{an})}^{M}, \theta^{ \pm a}, u^{ \pm i}\right), \\
x_{(\mathrm{an})}^{M} & =x^{M}+\frac{i}{2} \theta_{k}^{a} \gamma_{a b}^{M} \theta_{l}^{b} u^{+k} u^{-l}, \quad \theta^{ \pm a}=u_{k}^{ \pm} \theta^{a k} .
\end{aligned}
$$

A very important property of the analytic basis is that the set of coordinates

$$
\zeta:=\left(x_{(\mathrm{an})}^{M}, \theta^{+a}, u^{ \pm i}\right)
$$

involving only a half of the original Grassmann coordinates forms a subspace closed under the action of $\mathcal{N}=(1,0), 6 D$ supersymmetry. The set (3.15) parametrizes what is called "harmonic analytic superspace".

It is convenient to define the differential operators called spinor and harmonic derivatives. In the analytic basis, they are expressed as

$$
\begin{aligned}
D_{a}^{+} & =\partial_{-a}, D_{a}^{-}=-\partial_{+a}-2 i \theta^{-b} \partial_{a b}, \quad D^{0}=u^{+i} \frac{\partial}{\partial u^{+i}}-u^{-i} \frac{\partial}{\partial u^{-i}}+\theta^{+a} \partial_{+a}-\theta^{-a} \partial_{-a} \\
D^{++} & =\partial^{++}+i \theta^{+a} \theta^{+b} \partial_{a b}+\theta^{+a} \partial_{-a}, \quad D^{--}=\partial^{--}+i \theta^{-a} \theta^{-b} \partial_{a b}+\theta^{-a} \partial_{+a}
\end{aligned}
$$

where $\partial_{ \pm a} \theta^{ \pm b}=\delta_{a}^{b}$ and

$$
\partial^{++}=u^{+i} \frac{\partial}{\partial u^{-i}}, \quad \partial^{--}=u^{-i} \frac{\partial}{\partial u^{+i}} .
$$

The following commutation relations hold

$$
\begin{array}{rlrl}
\left\{D_{a}^{+}, D_{b}^{-}\right. & =2 i \partial_{a b}, & {\left[D^{++}, D^{--}\right]} & =D^{0}, \\
{\left[D^{++}, D_{a}^{+}\right]} & =\left[D^{--}, D_{a}^{-}\right]=0, \quad\left[D^{++}, D_{a}^{-}\right]=D_{a}^{+}, \quad\left[D^{--}, D_{a}^{+}\right]=D_{a}^{-} .
\end{array}
$$

\footnotetext{
${ }^{6}$ The constraint $u^{+i} u_{i}^{-}=1$ leaves in $u_{i}^{ \pm} 3$ degrees of freedom. One more degree of freedom is neutralized due to the strict preservation of the harmonic $U(1)$ charge in all invariant actions in the harmonic superspace.
} 
We shall use the notation

$$
\begin{aligned}
\left(D^{ \pm}\right)^{4} & =-\frac{1}{24} \varepsilon^{a b c d} D_{a}^{ \pm} D_{b}^{ \pm} D_{c}^{ \pm} D_{d}^{ \pm}, & \left(D^{ \pm}\right)^{3 a} & =-\frac{1}{6} \varepsilon^{a b c d} D_{b}^{ \pm} D_{c}^{ \pm} D_{d}^{ \pm}, \\
\left(\theta^{ \pm}\right)^{4} & =-\frac{1}{24} \varepsilon_{a b c d} \theta^{ \pm a} \theta^{ \pm b} \theta^{ \pm c} \theta^{ \pm d}, & \left(D^{ \pm}\right)^{4}\left(\theta^{\mp}\right)^{4} & =1,
\end{aligned}
$$

and the following conventions for the full and analytic superspace integration measures:

$$
d Z_{(\text {an })}=d^{6} x_{(\text {an })} d u\left(D^{-}\right)^{4}\left(D^{+}\right)^{4}, \quad d \zeta^{(-4)}=d^{6} x_{(\text {an })} d u\left(D^{-}\right)^{4} .
$$

The measure $d Z_{(\text {an) }}$ has canonical dimension -2 and $d \zeta^{(-4)}$ - dimension -4 . In what follows we will frequently suppress the subscript "(an)" of the analytic basis coordinate $x$ and the integration measure.

The harmonic integrals $\int F d u$ are nonzero only if the integrand $F$ has zero harmonic charge, $D^{0} F=0$. They can be computed using the rules

$$
\begin{aligned}
\int d u u_{j}^{+} u_{k}^{-} & =\frac{1}{2} \epsilon_{j k}, \\
\int d u u_{j}^{+} u_{k}^{+} u_{m}^{-} u_{n}^{-} & =\frac{1}{6}\left(\epsilon_{j m} \epsilon_{k n}+\epsilon_{j n} \epsilon_{k m}\right), \\
\int d u u_{j}^{+} u_{k}^{+} u_{m}^{+} u_{n}^{-} u_{l}^{-} u_{p}^{-} & =\frac{1}{24}\left(\epsilon_{j n} \epsilon_{k l} \epsilon_{m p}+5 \text { more terms }\right),
\end{aligned}
$$

etc.

\subsection{Superfields}

A general $6 D$ superfield depends on 8 odd coordinates $\theta_{i}^{a}$ (or $\theta^{ \pm a}$ ), which makes their component expansion rather complicated. There is, however, an important class of superfields, Grassmann-analytic superfields, which are defined on the analytic superspace (3.15) and so depend only on the half of the original Grassmann coordinates. The structure of Grassmann-analytic (G-analytic) superfields is much simpler than that of a general superfield.

A G-analytic superfield $\phi(\zeta)$ satisfies the constraint $D_{a}^{+} \phi=0 .^{7}$ In the analytic basis, $D_{a}^{+}$is reduced to the partial derivative $\partial / \partial \theta^{-a}$ and this constraint simply means that $\phi$ lives in the superspace (3.15).

The superfields can be classified according to their harmonic charge $q$, the eigenvalue of $D^{0}$. The pure $6 D$ SYM theory is formulated in terms of the G-analytic anti-Hermitian superfield gauge potential which has charge +2 and is denoted $V^{++}$. It defines the covariant harmonic derivative

$$
\nabla^{++}=D^{++}+V^{++}, \quad \delta V^{++}=-\nabla^{++} \Lambda
$$

where $\Lambda=\Lambda(\zeta)$ is an arbitrary analytic gauge parameter in the adjoint representation of the gauge group. It is convenient to introduce also a non-analytic gauge connection $V^{--}$ which covariantizes the harmonic derivative $D^{--}$

$$
\nabla^{--}=D^{--}+V^{--}, \quad \delta V^{--}=-\nabla^{--} \Lambda .
$$

\footnotetext{
${ }^{7}$ It is quite analogous to the habitual chirality constraint $D_{\alpha} \phi=0$ in four dimensions.
} 
Requiring $\nabla^{++}$and $\nabla^{--}$to satisfy the same algebra as their flat counterparts,

$$
\left[\nabla^{++}, \nabla^{--}\right]=D^{0},
$$

implies the harmonic zero-curvature condition

$$
D^{++} V^{--}-D^{--} V^{++}+\left[V^{++}, V^{--}\right]=0 .
$$

It can be used to solve for $V^{--}$in terms of $V^{++}$as a series over products of $V^{++}$taken at different harmonic "points",

$$
V^{--}(z, u)=\sum_{n=1}^{\infty}(-1)^{n} \int d u_{1} \ldots d u_{n} \frac{V^{++}\left(z, u_{1}\right) \ldots V^{++}\left(z, u_{n}\right)}{\left(u^{+} u_{1}^{+}\right)\left(u_{1}^{+} u_{2}^{+}\right) \ldots\left(u_{n}^{+} u^{+}\right)} .
$$

Here, the factors $\left(u^{+} u_{1}^{+}\right)^{-1}$, etc are the harmonic distributions [13] and the central basis coordinates $z$ are defined in (3.11).

For further use, note the following tensor relation between arbitrary variations of harmonic connections:

$$
\delta V^{--}=\frac{1}{2}\left(\nabla^{--}\right)^{2} \delta V^{++}-\frac{1}{2} \nabla^{++}\left(\nabla^{--} \delta V^{--}\right) .
$$

It follows from

$$
\nabla^{--} \delta V^{++}=\nabla^{++} \delta V^{--}
$$

which in turn follows from (3.26).

The connection $V^{--}$can be used to build up spinor and vector superfield connections,

$$
\mathcal{A}_{a}^{-}(V)=-D_{a}^{+} V^{--}, \quad \mathcal{A}_{a b}(V)=\frac{i}{2} D_{a}^{+} D_{b}^{+} V^{--},
$$

and the corresponding covariant spinor and vector derivatives,

$$
\begin{aligned}
\nabla_{a}^{-} & =D_{a}^{-}+\mathcal{A}_{a}^{-}, & \nabla_{a b} & =\partial_{a b}+\mathcal{A}_{a b}, \\
\delta \mathcal{A}_{a}^{-} & =-\nabla_{a}^{-} \Lambda, & \delta \mathcal{A}_{a b} & =-\nabla_{a b} \Lambda .
\end{aligned}
$$

The covariant derivatives (3.23), (3.24), (3.31) and $\nabla_{a}^{+}$(in the G-analytic basis, it keeps its flat form $D_{a}^{+}=\partial_{-a}$.) obey the same (anti)commutation relations (3.18) as the flat ones,

$$
\begin{aligned}
{\left[\nabla^{--}, D_{a}^{+}\right] } & =\nabla_{a}^{-}, \quad\left[\nabla^{++}, \nabla_{a}^{-}\right]=D_{a}^{+}, \quad\left[\nabla^{++}, D_{a}^{+}\right]=\left[\nabla^{--}, \nabla_{a}^{-}\right]=0, \\
{\left[D_{a}^{+}, \nabla_{b}^{-}\right] } & =2 i \nabla_{a b} .
\end{aligned}
$$

In addition,

$$
\left[\nabla^{++}, \nabla_{a b}\right]=0 .
$$

On the other hand, the commutators of spinor covariant derivatives with $\nabla_{a b}$ do not vanish,

$$
\left[D_{a}^{+}, \nabla_{b c}\right]=\frac{i}{2} \varepsilon_{a b c d} W^{+d}, \quad\left[\nabla_{a}^{-}, \nabla_{b c}\right]=\frac{i}{2} \varepsilon_{a b c d} W^{-d},
$$


where $W^{ \pm a}$ are the covariant $(1,0)$ spinor superfield strengths,

$$
\begin{aligned}
W^{+a} & =-\frac{1}{6} \varepsilon^{a b c d} D_{b}^{+} D_{c}^{+} D_{d}^{+} V^{--}, \\
W^{-a} & :=\nabla^{--} W^{+a} .
\end{aligned}
$$

One can also define the G-analytic superfield

$$
F^{++}=\frac{1}{4} D_{a}^{+} W^{+a}=\left(D^{+}\right)^{4} V^{--}, \quad D_{a}^{+} F^{++}=0 .
$$

From the harmonic zero-curvature condition (3.26) and G-analyticity of $V^{++}$, the important properties follow

$$
\begin{aligned}
\nabla^{++} W^{+a} & =\nabla^{--} W^{-a}=0, \quad \nabla^{++} W^{-a}=W^{+a}, \\
D_{b}^{+} W^{+a} & =\delta_{b}^{a} F^{++}, \\
\nabla^{++} F^{++} & =0 .
\end{aligned}
$$

Note that all these objects are homogeneously transformed by the gauge group

$$
\delta W^{ \pm a}=-\left[W^{ \pm a}, \Lambda\right], \quad \delta F^{++}=-\left[F^{++}, \Lambda\right] .
$$

More details on the algebra of gauge-covariant derivatives and the relevant Bianchi identities are collected in appendix A.

The matter hypermultiplet is described by a pair of the G-analytic superfields $q^{+A}(\zeta)$, $A=1,2$. If they belong to the real representation of the gauge group (e.g. the adjoint one), they can be subjected to the reality condition $\widetilde{q^{+A}}=\epsilon_{A B} q^{+B}$, where the ${ }^{\sim}$ conjugation is the product of the ordinary complex conjugation and an antipodal map on the harmonic sphere $S^{2} \sim \mathrm{SU}(2) / \mathrm{U}(1)$ (see [13] for details). Note that the gauge prepotentials $V^{ \pm \pm}$ defined above, as well as the connections $\mathcal{A}_{a}^{-}, \mathcal{A}_{a b}$ and covariant strengths $W^{ \pm a}$, are antiHermitian with respect to this generalized conjugation. Since, in what follows, we will deal with the adjoint hypermultiplets, it is worth to give how such $q^{+A}$ are transformed under the gauge group

$$
\delta q^{+A}=-\left[q^{+A}, \Lambda\right] .
$$

Finally, we note the useful Lemma:

$$
\nabla^{++} F^{-n}=0 \quad \Rightarrow \quad F^{-n}=0 \text { for } n \geq 1
$$

where the $\mathcal{N}=(1,0)$ superfield $F^{-n}$ transforms in some representation of the gauge group and we suppressed the "color" indices. This statement can be proved by passing to the central basis of the harmonic superspace, where $D^{++}=\partial^{++}$, and the so called $\tau$-frame for the gauge fields, where $\nabla^{++}=e^{-i V} \partial^{++} e^{i V}$ and $V$ is a harmonic superfield taking values in the algebra of the gauge group generators in the given representation and called "bridge". For the superfield $\tilde{F}^{-n}:=e^{i V} F^{-n}$ the constraint in (3.43) implies $\partial^{++} \tilde{F}^{-n}=0$, whence $\tilde{F}^{-n}=0$ (see eqs. (4.20), (4.21) in [13]) and $F^{-n}=0$. Note also that the constraint $\nabla^{++} F^{+n}=0, n \geq 0$, implies $F^{+n}=e^{-i V} F^{i_{1} \cdots i_{n}} u_{i_{1}}^{+} \cdots u_{i_{n}}^{+}$. This property will be widely used in section 7 . 


\section{Invariant actions of the $\mathcal{N}=(1,0)$ vector multiplet and a hyper- multiplet}

In this section we present the actions of canonical dimension $d=4$ containing the standard kinetic terms of the $\mathcal{N}=(1,0)$ vector gauge multiplet and a hypermultiplet.

\subsection{The dimension 4 Lagrangian of the gauge multiplet}

The superfield action providing the supersymmetric extension of the standard $d=4$ YangMills Lagrangian for the $6 D$ gauge fields $\sim \operatorname{Tr}\left(F^{M N} F_{M N}\right)$ is given by the following expression which is non-local in harmonics [15],

$$
S^{\mathrm{SYM}}=\frac{1}{f^{2}} \sum_{n=2}^{\infty} \frac{(-1)^{n}}{n} \operatorname{Tr} \int d^{6} x d^{8} \theta d u_{1} \ldots d u_{n} \frac{V^{++}\left(z, u_{1}\right) \ldots V^{++}\left(z, u_{n}\right)}{\left(u_{1}^{+} u_{2}^{+}\right) \ldots\left(u_{n}^{+} u_{1}^{+}\right)}
$$

where $f$ is a coupling constant carrying the dimension of inverse mass. This action is invariant under the supergauge transformations (recall (3.23), (3.24))

$$
\delta V^{++}=-\nabla^{++} \Lambda
$$

The gauge freedom (4.2) allows one to bring the superfield $V^{++}$in the Wess-Zumino gauge,

$$
V^{++}=\theta^{+a} \theta^{+b} A_{a b}+2\left(\theta^{+}\right)_{a}^{3} \lambda^{-a}-3\left(\theta^{+}\right)^{4} \mathcal{D}^{--},
$$

where $A_{a b}$ is the gauge field, $\lambda^{-a}=\lambda^{a i} u_{i}^{-}$is the gaugino and $\mathcal{D}^{--}=\mathcal{D}^{i k} u_{i}^{-} u_{k}^{-}$, where $\mathcal{D}^{i k}=\mathcal{D}^{k i}$ are the auxiliary fields. The component fields entering (4.3) depend only on the coordinates $x^{M}$, but not on the harmonic variables.

The component Lagrangian derived from (4.1) has a simple form,

$$
\mathcal{L}=\frac{1}{2 f^{2}} \operatorname{Tr}\left(-F_{M N}^{2}+i \lambda^{k} \gamma^{M} \nabla_{M} \lambda_{k}-\mathcal{D}^{i k} \mathcal{D}_{i k}\right)
$$

with $F_{M N}=\partial_{M} A_{N}-\partial_{N} A_{M}-i\left[A_{M}, A_{N}\right]$ and $\nabla_{M}=\partial_{M}-i A_{M}$. It gives rise to the standard equations of motion of the second order for the gauge fields and of the first order for the fermions.

These equations can be derived from the superfield equation of motion following from (4.1) by using the general formula for variation of $S^{\mathrm{SYM}}$,

$$
\delta S^{\mathrm{SYM}}=\frac{1}{f^{2}} \operatorname{Tr} \int d Z \delta V^{++} V^{--}=\frac{1}{f^{2}} \operatorname{Tr} \int d \zeta^{(-4)} \delta V^{++} F^{++} .
$$

This gives the extremely simple equation of motion

$$
F^{++}=0
$$




\subsection{The dimension 4 hypermultiplet Lagrangian}

The invariant action for the hypermultiplet in the adjoint representation (being interested in $\mathcal{N}=(1,1)$ extension, we will deal only with this assignment of the hypermultiplet), giving rise to the Lagrangian of the canonical dimension 4, is given by the following integral over the analytic superspace

$$
S^{q}=-\frac{1}{2 f^{2}} \operatorname{Tr} \int d \zeta^{(-4)} q^{+A} \nabla^{++} q_{A}^{+}, \quad \nabla^{++} q_{A}^{+}=D^{++} q_{A}^{+}+\left[V^{++}, q_{A}^{+}\right] .
$$

The coupling constant can be chosen the same as in $S^{\mathrm{SYM}}$, keeping in mind a freedom of rescaling of $q^{+A}$. The corresponding equation of motion is

$$
E^{+3}:=\nabla^{++} q_{A}^{+}=0 .
$$

An equivalent form of the same equation is

$$
\left(\nabla^{--}\right)^{2} q^{+A}=\nabla^{--} q^{-A}=0, \quad q^{-A}:=\nabla^{--} q^{+A} .
$$

This can be proved by acting on the l.h.s. of (4.9) by $\nabla^{++}$, observing that the result is zero as a consequence of (4.8), and then applying the Lemma of the previous section. Note also the useful relations

$$
D_{a}^{+} q^{-A}=-\nabla_{a}^{-} q^{+A}, \quad \nabla_{a}^{-} q^{-A}=0,
$$

which follow from the analyticity of $q^{+A}$ and the equations of motion (4.9).

As an instructive example, we consider the superfield action of the free hypermultiplet

$$
S_{\text {free }}^{q}=\int d \zeta^{(-4)} q^{+A} D^{++} q_{A}^{+} .
$$

The corresponding equation of motion is

$$
D^{++} q^{+A}=0 .
$$

The on-shell constraint (4.12) together with the G-analyticity condition $D_{a}^{+} q^{+A}=0$ can be resolved to find

$$
q^{+A}=\varphi^{+A}-\theta^{+a} \psi_{a}^{A}-i \theta^{+a} \theta^{+b} \partial_{a b} \varphi^{-A},
$$

where $\varphi^{ \pm A}=\varphi^{j A} u_{j}^{ \pm}$are physical harmonic-independent on-shell scalar fields. They satisfy the free equation of motion $\square \varphi=0$. And $\psi_{a}^{A}$ are right-handed on-shell fermionic fields satisfying the free Dirac equation.

\subsection{The $\mathcal{N}=(1,1)$ SYM action and its hidden $\mathcal{N}=(0,1)$ supersymmetry}

We now consider the actions (4.1) and (4.7) together and write

$$
S^{V q^{+}}=S^{\mathrm{SYM}}+S^{q}=\frac{1}{f^{2}}\left(\int d Z \mathcal{L}^{\mathrm{SYM}}-\frac{1}{2} \operatorname{Tr} \int d \zeta^{(-4)} q^{+A} \nabla^{++} q_{A}^{+}\right) .
$$


The sum (4.14) exhibits invariance under the extra hidden $\mathcal{N}=(0,1)$ supersymmetry,

$$
\delta_{0} V^{++}=\epsilon^{+A} q_{A}^{+}, \quad \delta_{0} q^{+A}=-\left(D^{+}\right)^{4}\left(\epsilon_{A}^{-} V^{--}\right), \quad \epsilon_{A}^{ \pm}=\epsilon_{a A} \theta^{ \pm a},
$$

which completes the manifest $\mathcal{N}=(1,0)$ supersymmetry to $\mathcal{N}=(1,1)$. This means that (4.14) is in fact the $\mathcal{N}=(1,0)$ form of the $\mathcal{N}=(1,1)$ SYM theory action. Note a useful representation for the variation $\delta_{0} q^{+A}$ through the superfield strengths $F^{++}$and $W_{a}^{+}$:

$$
\delta_{0} q_{A}^{+}=-\epsilon_{a A}\left(\theta^{-a} F^{++}-W^{+a}\right) .
$$

It is consistent with the analyticity of $q^{+A}$ because of the analyticity of $F^{++}$and the relation (3.39).

The invariance (4.15) is quite analogous to the hidden $\mathcal{N}=2,4 D$ supersymmetry which completes the manifest $\mathcal{N}=2,4 D$ supersymmetry of the sum of the harmonic superspace actions for the $\mathcal{N}=2,4 D$ SYM field and the adjoint hypermultiplet to $\mathcal{N}=4$ supersymmetry [13]. This sum is thus nothing but a representation of the $\mathcal{N}=4,4 D$ SYM action in terms of $\mathcal{N}=2$ superfields.

Like in the $\mathcal{N}=4,4 D$ case, the transformations (4.15) have the correct closure with themselves and with the manifest $\mathcal{N}=(1,0)$ supersymmetry only on mass shell, when the equations of motion corresponding to the action (4.14),

$$
E^{++}:=F^{++}+\frac{1}{2}\left[q^{+A}, q_{A}^{+}\right]=0, \quad E^{+3}:=\nabla^{++} q_{A}^{+}=0,
$$

are satisfied.

A direct calculation shows that $\left(\delta_{2} \delta_{1}-\delta_{1} \delta_{2}\right) V^{++}$amounts to

$$
\left(\delta_{2} \delta_{1}-\delta_{1} \delta_{2}\right) V^{++}=-\nabla^{++} \Lambda+i \varepsilon^{a b c d} f_{21[a b]} \partial_{c d} V^{++}, \quad f_{21[a b]}:=\epsilon_{2[a}^{A} \epsilon_{1 b] A},
$$

where

$$
\Lambda=\left(D^{+}\right)^{4}\left[\left(\epsilon_{1}^{-A} \epsilon_{2 A}^{-}\right) V^{--}\right]
$$

is a gauge transformation superfield parameter. Thus, the hidden supersymmetry has the correct off-shell closure on $V^{++}$. This is not the case for $q_{A}^{+}$. The same bracket yields

$$
\left(\delta_{2} \delta_{1}-\delta_{1} \delta_{2}\right) q_{A}^{+}=\left[\Lambda, q_{A}^{+}\right]+i \varepsilon^{a b c d} f_{21[a b]} \partial_{c d} q_{A}^{+}-\left(D^{+}\right)^{4}\left[\epsilon_{2 A}^{-} \tilde{\delta}_{1} V^{--}-\left[\epsilon_{1 A}^{-} \tilde{\delta}_{2} V^{--}\right],\right.
$$

where $\tilde{\delta} V^{--}$is defined from the relation

$$
\delta_{0} V^{--}=\epsilon^{-A} \nabla^{--} q_{A}^{+}+\tilde{\delta} V^{--},
$$

and involves the terms vanishing on the hypermultiplet equations of motion (4.8), (4.9). Thus, in the full analogy with $(2.4),\left(\delta_{2} \delta_{1}-\delta_{1} \delta_{2}\right) q^{+A}$ involves a nontrivial extra term which vanishes only on mass shell.

Let us now consider the commutators of the hidden supersymmetry with the manifest one, i.e. with

$$
\hat{\delta}\left(q_{A}^{+}, V^{++}\right)=\eta^{+a} Q_{a}^{-}\left(q_{A}^{+}, V^{++}\right) \quad Q_{a}^{-}=\frac{\partial}{\partial \theta^{+a}}+\ldots, \quad \eta^{+a}=\eta^{i a} u_{i}^{+} .
$$


We find

$$
\left(\hat{\delta} \delta_{0}-\delta_{0} \hat{\delta}\right) V^{++}=\left(\eta^{i a} \epsilon_{a}^{B} u_{i}^{+}\right) q_{B}^{+}:=f^{B i} u_{i}^{+} q_{B}^{+} .
$$

This variation can be identically rewritten as

$$
f^{B i} u_{i}^{+} q_{B}^{+}=\nabla^{++}\left(f^{B i} u_{i}^{-} q_{B}^{+}\right)-f^{B i} u_{i}^{-} \nabla^{++} q_{B}^{+},
$$

i.e., once again, it is reduced to some analytic gauge transformation of $V^{++}$only on the hypermultiplet mass shell, i.e., with $\nabla^{++} q^{+A}=0$.

Analogously,

$$
\left(\hat{\delta} \delta_{0}-\delta_{0} \hat{\delta}\right) q_{A}^{+}=-\left[f^{B i} u_{i}^{-} q_{B}^{+}, q_{A}^{+}\right]-f_{A}^{i} u_{i}^{-}\left(F^{++}+\frac{1}{2}\left[q^{+C}, q_{C}^{+}\right]\right),
$$

i.e., it is reduced to the pure gauge transformation on the mass shell for $V^{++}$.

We conclude that the correct $\mathcal{N}=(1,1)$ closure of the transformations (4.15) with themselves and with the manifest $\mathcal{N}=(1,0)$ transformations is achieved only on the mass shell for both superfields $V^{++}$and $q^{+A}$. To avoid a possible confusion, we point out that the action (4.14) is invariant under the transformations (4.15) off shell, with $V^{++}$and $q^{+A}$ being unconstrained analytic superfields. The mass-shell conditions are required to ensure the correct $\mathcal{N}=(1,1)$ closure for these transformations.

It is instructive to see how the superfield equations of motion (4.17) are transformed into each other under the transformations (4.15). Using the properties (3.40), we find

$$
\delta_{0} E_{A}^{+3}=-\epsilon_{A}^{+} E^{++} .
$$

It is a little more complicated to see that the variation of $E^{++}$is actually expressed through $E^{+3}$ and the alternative form (4.9) of the hypermultiplet equation of motion (4.8). It is easy to show that

$$
\delta_{0} V^{--}=\epsilon^{-A} \nabla^{--} q_{A}^{+}+\text {terms containing }\left(\nabla^{--}\right)^{2} q^{+B} \text { and } \nabla^{++} q^{+B} .
$$

Then it can be shown that the contribution of the first "dangerous" term in (4.27) to $\delta_{0} F^{++}$ is exactly canceled by the variation of the second term in $E^{++}$, so $\delta_{0} E^{++}$is expressed through the terms containing the equivalent forms (4.9) of the hypermultiplet equation of motion.

In what follows we will meet the situation when the superfields involved in the $\mathcal{N}=(0,1)$ transformations above satisfy themselves the mass-shell equations (4.17), (4.8) or (4.9). The various superfields defined earlier are transformed on shell as

$$
\begin{aligned}
\delta q^{ \pm A} & =\hat{\delta} q^{ \pm A}-\left[\epsilon^{-B} q_{B}^{+}, q^{ \pm A}\right], & \hat{\delta} q^{ \pm A} & =\epsilon_{a}^{A} W^{ \pm a}, \\
\delta W^{ \pm a} & =\hat{\delta} W^{ \pm a}-\left[\epsilon^{-B} q_{B}^{+}, W^{ \pm a}\right], & \hat{\delta} W^{ \pm a} & =-i \varepsilon^{a b c d} \epsilon_{b}^{A} \nabla_{c d} q_{A}^{ \pm} .
\end{aligned}
$$

Note that the transformation (4.28) immediately follows from (4.16) upon using the equation of motion $E^{++}=0$ from the set (4.17). 

of $q^{ \pm A}$ :

We will need also the on-shell transformation rules for the spinor and vector derivatives

$$
\begin{aligned}
& \delta\left(\nabla_{b c} q_{A}^{ \pm}\right)=\epsilon_{a A} \nabla_{b c} W^{ \pm a}+\frac{i}{2}\left(\epsilon_{b}^{B}\left[\nabla_{c}^{-} q_{B}^{+}, q_{A}^{ \pm}\right]-\epsilon_{c}^{B}\left[\nabla_{b}^{-} q_{B}^{+}, q_{A}^{ \pm}\right]\right)-\left[\epsilon^{-B} q_{B}^{+}, \nabla_{b c} q_{A}^{ \pm}\right], \\
& \delta\left(D_{a}^{+} q_{A}^{-}\right)=-\delta\left(\nabla_{a}^{-} q_{A}^{+}\right)=-\epsilon_{b A} D_{a}^{+} W^{-b}+\epsilon_{a}^{B}\left[q_{B}^{+}, q_{A}^{-}\right]-\left[\epsilon^{-B} q_{B}^{+}, D_{a}^{+} q_{A}^{-}\right] .
\end{aligned}
$$

Note that the last terms in the variations (4.28)-(4.31) are some field-dependent gauge transformations and they do not contribute to the variation of the gauge-invariant Lagrangian involving the traces over the "color" indices.

\section{Higher-dimensional $\mathcal{N}=(1,0)$ and $\mathcal{N}=(1,1)$ invariants}

Now we turn to the discussion of higher-dimensional invariants. As was mentioned in the very beginning, the pure $6 D$ gauge theories are chiral theories, they involve only the left-handed gaugino field and hence are plagued by the chiral anomaly [1-4]. In other words, gauge symmetry is broken there by quantum effects, which restricts their physical interest. Anomaly can be canceled and the gauge symmetry kept intact in the theories involving, besides the left-handed gauginos, also right-handed fermions belonging to the matter hypermultiplet. This condition is obviously satisfied in the $\mathcal{N}=(1,1)$ gauge theory. We will be mainly interested in this section in the on-shell $\mathcal{N}=(1,1)$ invariant gauge theory, which is written in terms of $\mathcal{N}=(1,0)$ superfields and may or may not possess the full off-shell $\mathcal{N}=(1,0)$ supersymmetry.

For a higher-dimensional operator to be a counterterm giving a logarithmically divergent contribution to the scattering amplitudes $\sim \ln \Lambda_{U V}$, it must not vanish on mass shell, but its supersymmetric variation under the on-shell $\mathcal{N}=(1,1)$ transformations should be reduced to a total derivative. We first discuss the operators of canonical dimension 6 .

\section{$5.1 d=6$}

It is very easy to write down the superfield gauge-invariant action of canonical physical dimension 6 in the gauge field sector. It has the following unique form [40]:

$$
S_{\mathrm{SYM}}^{(6)}=\frac{1}{2 g^{2}} \operatorname{Tr} \int d \zeta^{(-4)} d u\left(F^{++}\right)^{2} .
$$

Here the coupling constant $g$ is dimensionless. ${ }^{8}$ The component expression for (5.1) involves extra derivatives,

$$
S=-\frac{1}{2 g^{2}} \operatorname{Tr} \int\left[\left(\nabla^{M} F_{M L}\right)^{2}+\frac{1}{2}\left(\nabla_{M} \mathcal{D}_{j k}\right)^{2}+\mathcal{D}_{l k} \mathcal{D}^{k j} \mathcal{D}_{j}^{l}+\text { fermion terms }\right] .
$$

We see that the auxiliary fields of the Lagrangian (4.4) enter the $d=6$ Lagrangian with derivatives - the same phenomenon that we observed in section 2 in a toy SQM model;

\footnotetext{
${ }^{8}$ Indeed, the superfields $V^{++}, V^{--}$are dimensionless, and it follows that $F^{++}$defined in (3.37) has canonical dimension 2.
} 
the higher-dimensional Lagrangian (5.2) is related to the Lagrangian (4.4) in the same way as the higher-dimensional Lagrangian (2.10) to the Witten Lagrangian (2.1).

We observe that the integrand in (5.1) is just the square of the equation of motion (4.6) and therefore this $d=6$ action vanishes on mass shell modulo possible hypermultiplet terms. Now we are going to show that the same remains true for the $d=6$ actions taking into account the hypermultiplet terms.

One can write a series of new hypermultiplet $d=6$ actions $S_{n}$ representing full superspace integrals [41],

$$
S_{n} \sim \operatorname{Tr} \int d u d Z q^{+A}\left(\nabla^{--}\right)^{n}\left(\nabla^{++}\right)^{n-1} q_{A}^{+} .
$$

All the terms with $n>1$ vanish on mass shell. Using (3.37), it is convenient to represent the non-vanishing action $S_{1}$ as an integral over the analytic superspace,

$$
\operatorname{Tr} \int d Z q^{+A} \nabla^{--} q_{A}^{+}=\operatorname{Tr} \int d \zeta^{(-4)} F^{++}\left[q_{A}^{+}, q^{+A}\right] .
$$

There is one more $d=6$ interaction involving the hypermultiplet field. It does not contain harmonic derivatives and is given by the analytic superspace integral,

$$
S_{\text {quart }} \sim \operatorname{Tr} \int d u d \zeta^{(-4)}\left[q^{+A}, q_{A}^{+}\right]^{2}
$$

Thus, if disregarding the terms vanishing on the mass shell, a generic $\mathcal{N}=(1,0)$ invariant Lagrangian reads

$$
\mathcal{L}^{d=6}=\frac{1}{2 g^{2}} \operatorname{Tr} \int d u d \zeta^{(-4)}\left[\left(F^{++}\right)^{2}+\alpha F^{++}\left[q^{+A}, q_{A}^{+}\right]+\beta\left[q^{+A}, q_{A}^{+}\right]^{2}\right] .
$$

The requirement that its $\mathcal{N}=(0,1)$ variation vanishes on mass shell imposes the restriction $\alpha=2 \beta+1 / 2$ such that the Lagrangian acquires the form

$$
\mathcal{L}^{d=6}=\frac{1}{2 g^{2}} \operatorname{Tr} \int d u d \zeta^{(-4)}\left(F^{++}+\frac{1}{2}\left[q^{+A}, q_{A}^{+}\right]\right)\left(F^{++}+2 \beta\left[q^{+A}, q_{A}^{+}\right]\right),
$$

which vanishes on shell due to (4.17).

We have thus shown that the non-vanishing on-mass-shell counterterms of canonical dimension 6 are absent, and this proves the one-loop finiteness of the theory (4.14).

The fact that the algebra of extended supertransformations does not close off shell suggests that an action corresponding to (5.7) with some fixed $\beta$, to which a series of the actions $S_{n}$ in (5.3) with arbitrary coefficients is added, cannot be invariant off shell. Indeed, when one tries to construct such an invariant (the corresponding calculations are presented in appendix B), one meets obstacles that seem to be unsurmountable. It is easy to see that, in order to ensure the cancelation of the terms $\propto\left(q^{+}\right)^{3}$ in the variation, the coefficient $\beta$ in (5.7) should be fixed to $\beta=1 / 4$. But the linear in $q^{+A}$ terms do not want to be canceled among themselves, no matter what you try.

We thus conjecture that a $d=6$ off-shell $\mathcal{N}=(1,1)$ supersymmetric invariant does not exist. ${ }^{9}$

\footnotetext{
${ }^{9}$ We did not rigourously prove it, however — it is always difficult to prove the absence of something.
} 


\section{$5.2 d=8$}

Once again, we begin with the gauge field sector and write appropriate off-shell $\mathcal{N}=(1,0)$ supersymmetric gauge invariants of canonical dimension $d=8$, having in mind to extend them to the $\mathcal{N}=(1,1)$ invariants by adding some hypermultiplet terms. It turns out that all such purely gauge field terms vanish on the mass shell of (4.4), in agreement with [10]. Then we write the full list of different possible $d=8, \mathcal{N}=(1,0)$ superfield terms involving the hypermultiplet contributions and demonstrate that, on the equations of motion corresponding to the total $\mathcal{N}=(1,1)$ action $(4.14)$, they are all reduced to a single expression, which is not invariant under the hidden supersymmetry (4.15), (4.28)(4.31) (and there is no way to make it invariant).

We consider first the $d=8$ terms in the pure gauge field sector. The SYM equations of motion are $F^{++}=0$. The vanishing of some structures (like $\operatorname{Tr} \int d Z \nabla_{a}^{-} W^{-a} F^{++}$), is obvious. We consider now a couple of less trivial examples.

- Let

$$
S_{1}^{(8)}=\operatorname{Tr} \int d Z\left(\nabla_{a}^{-} W^{+a}\right)\left(D_{a}^{+} W^{-a}\right),
$$

where $W^{-a}=\nabla^{--} W^{+a}$. We use the identity

$$
\nabla_{a}^{-} W^{+a}=D_{a}^{+} W^{-a},
$$

which is derived in appendix A as a corollary of certain Bianchi identities. We obtain

$$
S_{1}^{(8)}=\operatorname{Tr} \int d Z\left(\nabla_{a}^{-} W^{+a}\right)^{2}=\operatorname{Tr} \int d \zeta^{(-4)}\left(D^{+}\right)^{4}\left(\nabla_{a}^{-} W^{+a}\right)^{2} .
$$

The terms involving $\left(D^{+}\right)^{3}\left(\nabla_{a}^{-} W^{+a}\right)$ and $\left(D^{+}\right)^{4}\left(\nabla_{a}^{-} W^{+a}\right)$ contain $F^{++}$and vanish on mass shell. We are left with the structure

$$
\propto \epsilon^{\text {cdef }} D_{c}^{+} D_{d}^{+}\left(\nabla_{a}^{-} W^{+a}\right) D_{e}^{+} D_{f}^{+}\left(\nabla_{b}^{-} W^{+b}\right) .
$$

On mass shell, it is equivalent to

$$
\propto \epsilon^{\text {cdef }} \epsilon_{\text {cdam }}\left\{W^{+m}, W^{+a}\right\} \epsilon_{e f b n}\left\{W^{+n}, W^{+b}\right\} \sim \epsilon_{\text {manb }}\left\{W^{+m}, W^{+a}\right\}\left\{W^{+n}, W^{+b}\right\},
$$

which vanishes as the anticommutator $\left\{W^{+m}, W^{+a}\right\}$ is symmetric under $m \leftrightarrow a$.

- Let

$$
S_{2}^{(8)}=\operatorname{Tr} \int d Z\left(\nabla_{a}^{-} W^{+b}\right)\left(D_{b}^{+} W^{-a}\right) .
$$

Integrating by parts with respect to $\nabla_{a}^{-}$, using the commutation relation

$$
\left\{D_{b}^{+}, \nabla_{a}^{-}\right\}=2 i \nabla_{b a}=-2 i \nabla_{a b}=-\left\{D_{a}^{+}, \nabla_{b}^{-}\right\}
$$

disregarding the terms involving $F^{++}$, and integrating by parts once again, we reduce (5.11) to (5.8). 
Now we turn to the general proof that there exist no $\mathcal{N}=(1,0)$ supersymmetric offshell invariants of the dimension 8 which could respect the on-shell $\mathcal{N}=(1,1)$ invariance.

To this end, we construct the full set of the superfield Lagrangians of dimension 4 in the full $\mathcal{N}=(1,0)$ harmonic superspace (they correspond to the dimension 8 component Lagrangians): ${ }^{10}$

$$
\begin{aligned}
& L_{W}^{(1)}=\nabla_{a}^{-} W^{-a} D_{b}^{+} W^{+b}, L_{W}^{(2)}=\nabla_{a}^{-} W^{+a} D_{b}^{+} W^{-b}, \quad L_{W}^{(3)}=\nabla_{a}^{-} W^{+b} D_{b}^{+} W^{-a}, \\
& L_{W}^{(4)}=\nabla_{a}^{-} W^{+b} \nabla_{b}^{-} W^{+a}, L_{W}^{(5)}=D_{a}^{+} W^{-b} D_{b}^{+} W^{-a}, \\
& L_{q}^{(1)}=i S_{a}^{A} \nabla_{b c} S_{d A} \varepsilon^{a b c d}, L_{q}^{(2)}=\left[q^{+(A}, q^{-B)}\right]\left[q_{A}^{+}, q_{B}^{-}\right], L_{q}^{(3)}=\left[q^{-A}, q_{A}^{-}\right]\left[q^{+B}, q_{B}^{+}\right] .
\end{aligned}
$$

Note that a conceivable term $\sim W^{+a} \nabla_{a b} W^{-b}$ is reduced to the other structures in the list (5.13), (5.14) by integrating by parts with respect to the spinor derivatives under the (undisplayed) trace.

Using the off-shell relations (A.3), (A.4) and (5.9) and also bearing in mind that $\left\{D_{a}^{+}, D_{b}^{+}\right\}=\left\{\nabla_{a}^{-}, \nabla_{b}^{-}\right\}=0$, it is easy to show that all Lagrangians in the set (5.13) are reduced to $L_{W}^{(2)}$ or to $L_{W}^{(1)}$, which in turn are related to each other by integrating by parts with respect to $\nabla^{--}$. This proof is valid off shell and does not require passing to the analytic subspace at any intermediate step.

Next, using the on-shell relations (A.8), it is straightforward to show that

$$
L_{W}^{(1)}(\text { on-shell }) \Rightarrow 4 L_{q}^{(3)} .
$$

Also, using simple algebraic manipulations and integrating by parts with respect to harmonic derivatives, one can show that

$$
L_{q}^{(2)}(\text { on-shell }) \Rightarrow \frac{3}{4} L_{q}^{(3)} .
$$

It remains to work out $L_{q}^{(1)}$. Integrating by parts, it can be reduced to

$$
L_{q}^{(1)} \Rightarrow-i \varepsilon^{a b c d} q^{-A} D_{a}^{+} \nabla_{b c} D_{d}^{+} q_{A}^{-} .
$$

Using the on-shell relation (A.19) and, once again, integrating the term $q^{-A}\left\{W^{+a}, \nabla_{a}^{-} q_{A}^{+}\right\}$ by parts with respect to $\nabla_{a}^{-}$, one reduces $L_{q}^{(1)}$, up to a total harmonic derivative, to $2 L_{q}^{(3)}$.

Thus, all possible superfield Lagrangians of the dimension 4 are reduced on mass shell to the single non-vanishing structure

$$
L_{q}^{(3)}=\left[q^{-A}, q_{A}^{-}\right]\left[q^{+B}, q_{B}^{+}\right]
$$

Bearing in mind the overall trace, the variation of $L_{q}^{(3)}$ under the hidden $\mathcal{N}=(0,1)$ supersymmetry (4.28) is given by

$$
\delta_{\epsilon} L_{q}^{(3)} \sim \epsilon_{a A}\left[q^{-B}, q_{B}^{-}\right]\left[q^{+A}, W^{+a}\right] .
$$

\footnotetext{
${ }^{10}$ For brevity, we omit here the $\operatorname{Tr}$ symbol with respect to "color" indices, but we will always have it in mind.
} 
It is non-vanishing, and no terms can be invented to cancel (5.19). Thus, no $\mathcal{N}=(1,1)$ invariant terms of the dimension 8 can be constructed out of the $\mathcal{N}=(1,0)$ superfields.

It is worth noting that in the hypermultiplet sector one can contemplate $\mathcal{N}=(1,0)$ invariants which are not reduced to the product of "color" anticommutators as in (5.18), e.g.,

$$
\sim q^{+A} q_{A}^{-} q^{+B} q_{B}^{-}, \quad \text { or } \sim q^{+A} q^{-B} q_{A}^{+} q_{B}^{-} .
$$

Nevertheless, it is impossible to ensure the mutual cancelations of the $\mathcal{N}=(0,1)$ variations of such terms, while keeping the requirement for the Lagrangian not to vanish on mass shell. To check this, we wrote down all the independent terms of this kind, calculated their variations (reducing $\delta q^{-A}=\nabla^{--} \delta q^{+A}$ to $\delta q^{+A}$ through integrating by parts) and found the unique combination of such terms, $\sim \operatorname{Tr}\left(q^{+A} q_{A}^{-} q^{+B} q_{B}^{-}+q^{+A} q^{-B} q_{B}^{+} q_{A}^{-}\right)$, the variation of which is zero up to a total harmonic derivative. However, it is easy to show that, on the mass shell of $q^{+A}$, this combination is a total harmonic derivative on its own.

Surprisingly, the $d=8$ superfield expression which is non-vanishing on shell and respects the on-shell $\mathcal{N}=(1,1)$ supersymmetry can be constructed if we give up the requirement of off-shell $\mathcal{N}=(1,0)$ supersymmetry.

\subsection{On-shell $\mathcal{N}=(1,0)$ and $\mathcal{N}=(1,1)$ invariants}

As the complete off-shell $\mathcal{N}=(1,1)$ superfield formalism is absent, it is not possible to write down operators of a fixed canonical dimension $d>4$ which would be invariant off shell under the $\mathcal{N}=(1,1)$ transformations. This concerns the operators of dimension $d=8$ and $d=10$. However, in contrast to the case $d=6$, the on-shell $d=8$ and $d=10$ invariants exist, and it is possible to find them. The basic idea is to seek for the invariants, in which not only the hidden $\mathcal{N}=(0,1)$ supersymmetry is realized on shell, but which are $\mathcal{N}=(1,0)$ supersymmetric also only on shell.

Once again, we start our consideration from the simple example in the gauge field sector. If we lift the requirement of off-shell $\mathcal{N}=(1,0)$ supersymmetry, we can define the nonvanishing $d=8$ operators that are supersymmetric only on mass shell. One of them reads

$$
\tilde{S}_{1}^{(8)}=\frac{1}{4} \operatorname{Tr} \int d \zeta^{(-4)} \varepsilon_{a b c d} W^{+a} W^{+b} W^{+c} W^{+d},
$$

where the factor $\frac{1}{4}$ was introduced for further convenience. Indeed, eq. (3.39) tells us that $D_{a}^{+} W^{+b}=\delta_{a}^{b} F^{++}$, which vanishes on mass shell. Thus, when disregarding the terms proportional to the equations of motion, $W^{+a}$ is a G-analytic superfield and so the action (5.21) respects $\mathcal{N}=(1,0)$ supersymmetry on shell. ${ }^{11}$ Being expressed through components, the bosonic part of (5.21) gives the known $F^{4}$ structure [50],

$$
\begin{aligned}
{\left[\mathcal{L}_{\text {an }}^{(8)}\right]_{\text {bos }}=} & \frac{1}{2 \cdot 81} \operatorname{Tr}_{(s)}\left[2 F_{M N} F^{M N} F_{P Q} F^{P Q}+F_{M N} F_{P Q} F^{M N} F^{P Q}\right. \\
& \left.-4 F^{N M} F_{M R} F^{R S} F_{S N}-8 F^{N M} F_{M Q} F_{N R} F^{R Q}\right] .
\end{aligned}
$$

\footnotetext{
${ }^{11}$ Note that a similar on-shell invariant appears as a one-loop contribution to the quantum effective action of the $\mathcal{N}=(1,0)$ gauge theory in the $6 D$ harmonic superspace in a special background [49].
} 
This expression can be derived using the component representation for $W^{+a}[40,51]$,

$$
W^{+a}=\frac{i}{6} F_{M N}\left(\sigma^{M N}\right)_{b}^{a} \theta^{+b}+\text { fermion terms }+ \text { terms vanishing on shell }+O\left[\left(\theta^{+}\right)^{2}\right],
$$

as well as the identities (3.5), (3.6). Note the presence of the symmetrized color traces $\operatorname{Tr}_{(s)} \sim \operatorname{Tr} X(Y Z U+U Y Z+Z U Y)$ in (5.22).

This tensor structure reproduces indeed the so-called $t_{8}$ tensor obtained in the treelevel four-gluon scattering amplitude [50]. The complete component form of the associated supersymmetry invariant in six dimensions was first obtained in [43].

It is also possible to write down an on-shell $\mathcal{N}=(1,0)$ supersymmetric invariant involving the product of two color traces,

$$
\tilde{S}_{2}^{(8)}=\frac{1}{4} \int d \zeta^{(-4)} \varepsilon_{a b c d} \operatorname{Tr}\left(W^{+a} W^{+b}\right) \operatorname{Tr}\left(W^{+c} W^{+d}\right) .
$$

The next step is to seek for the on-shell $\mathcal{N}=(1,1)$ completion of the $d=8$ terms $(5.21)$ and (5.24). Clearly, it should be a collection of terms containing the hypermultiplet superfield $q^{+A}$. First one should construct the full list of the dimension $d=8$ operators which are G-analytic on the shell of the full set of equations of motion following from the action (4.14), i.e. eqs. (4.17) and (4.8) [or (4.9)]. Next one needs to select the $\mathcal{N}=(0,1)$ invariant combination of such operators (if it exists).

The minimal on-shell G-analytic extension of (5.21) [i.e. the expression analytic as a consequence of the full set of equations $(4.17),(4.8)]$ is given by the following expression:

$$
\begin{aligned}
L_{0}^{+4}= & \operatorname{Tr}\left\{\frac{1}{4} \varepsilon_{a b c d} W^{+a} W^{+b} W^{+c} W^{+d}-i \nabla_{a b} q^{+A}\left(W^{+a} q_{A}^{+} W^{+b}+2 q_{A}^{+} W^{+a} W^{+b}\right)\right. \\
& -W^{+a} D_{a}^{+} q^{-A}\left[q_{A}^{+}\left(q^{+}\right)^{2}+\frac{1}{2}\left(q^{+}\right)^{2} q_{A}^{+}\right]+\left(q^{+}\right)^{2} D_{a}^{+} q^{-A}\left(q_{A}^{+} W^{+a}+\frac{1}{2} W^{+a} q_{A}^{+}\right) \\
& \left.-2\left(q^{+}\right)^{2}\left[q^{-A} q_{A}^{+}\left(q^{+}\right)^{2}+\frac{1}{2} q^{-A}\left(q^{+}\right)^{2} q_{A}^{+}\right]\right\},
\end{aligned}
$$

where $\left(q^{+}\right)^{2}:=q^{+A} q_{A}^{+}=\frac{1}{2}\left[q^{+A}, q_{A}^{+}\right]$. The full list of other possible $d=8$ superfield Ganalytic terms involving the single trace is given in appendix C. It is shown there that, by integrating by parts, they all can be reduced to the two independent structures, $L_{2}^{+4}$ and $L_{3}^{+4}$ [eqs. (C.3) and (C.4)]. Then the $\mathcal{N}=(1,1)$ supersymmetric combination is uniquely determined to be

$$
\mathcal{L}_{(1,1)}^{+4}=L_{0}^{+4}+L_{3}^{+4} .
$$

It is instructive to see how the proof goes on in the abelian case. Passing to the abelian limit in (5.25) and (C.4), we write

$$
\begin{aligned}
\mathcal{L}_{(1,1)}^{+4} & =\frac{1}{4} \varepsilon_{a b c d} W^{+a} W^{+b} W^{+c} W^{+d}+3 i q^{+A} \partial_{a b} q_{A}^{+} W^{+a} W^{+b}-q^{+A} \partial_{a b} q_{A}^{+} q^{+B} \partial^{a b} q_{B}^{+} \\
& \equiv \mathcal{L}_{(I)}^{+4}+\mathcal{L}_{(I I)}^{+4}+\mathcal{L}_{(I I I)}^{+4} .
\end{aligned}
$$

Our task is to prove that it is invariant on mass shell under the transformations

$$
\delta q^{+A}=\epsilon_{a}^{A} W^{+a}, \quad \delta W^{+a}=-2 i \epsilon_{b}^{A} \partial^{a b} q_{A}^{+} .
$$


It is easy to see that the linear in $q$ terms in the sum of $\delta \mathcal{L}_{(I)}^{+4}$ and $\delta \mathcal{L}_{(I I)}^{+4}$ vanish. We are left with

$$
\Delta:=\delta \mathcal{L}_{(I)}^{+4}+\delta \mathcal{L}_{(I I)}^{+4}=6 \epsilon_{c}^{B} \varepsilon^{a c d e} q^{+A} \partial_{a b} q_{A}^{+} \partial_{d e} q_{B}^{+} W^{+b},
$$

The variation of $\mathcal{L}_{(I I I)}^{+4}$ is

$$
\delta \mathcal{L}_{(I I I)}^{+4}=-2 \epsilon_{c}^{B} \varepsilon^{a b e d} q^{+A} \partial_{a b} q_{A}^{+} \partial_{e d} q_{B}^{+} W^{+c} .
$$

To see the cancelation of (5.29) and (5.30), one should use the cyclic identities

$$
\begin{aligned}
\varepsilon^{a b c d} \delta_{f}^{e}+\varepsilon^{b c d e} \delta_{f}^{a}+\varepsilon^{c d e a} \delta_{f}^{b}+\varepsilon^{d e a b} \delta_{f}^{c}+\varepsilon^{e a b c} \delta_{f}^{d} & =0 \\
\varepsilon^{A B} \delta_{D}^{C}+\varepsilon^{B C} \delta_{D}^{A}+\varepsilon^{C A} \delta_{D}^{B} & =0
\end{aligned}
$$

and the equations of motion $\partial_{a b} W^{+b}=0, \square q^{+A}=0$.

Namely, we represent

$$
\Delta=-6 \epsilon_{c}^{B} q^{+A} \partial_{a b} q_{A}^{+} \partial_{d e} q_{B}^{+} W^{+f}\left[\varepsilon^{c d e b} \delta_{f}^{a}+\varepsilon^{d e b a} \delta_{f}^{c}+\varepsilon^{e b a c} \delta_{f}^{d}+\varepsilon^{b a c d} \delta_{f}^{e}\right] \equiv \mathcal{A}+\mathcal{B}+\mathcal{C}+\mathcal{D}
$$

and then observe that $\mathcal{A}=-\Delta, \mathcal{B}=-3 \delta \mathcal{L}_{(I I I)}^{+4}$ and

$$
\mathcal{C}=\mathcal{D}=-6 \epsilon_{c}^{B} \varepsilon^{a b c e} q^{+A} \partial_{a b} q_{A}^{+} \partial_{d e} q_{B}^{+} W^{+d} .
$$

Next, using the second identity in (5.31) and integrating by parts, we derive that (on shell !) $\mathcal{C}=-\Delta-\mathcal{C}$ and hence $\mathcal{C}=-\Delta / 2$. This gives

$$
\Delta=-\Delta-3 \delta \mathcal{L}_{(I I I)}^{+4}-\frac{1}{2} \Delta-\frac{1}{2} \Delta,
$$

and, finally, $\Delta=-\delta \mathcal{L}_{(I I I)}^{+4}$.

The proof in the non-abelian case is much more complicated since there is a lot of various terms coming from different sources. Nevertheless, we checked that (5.26) is still invariant up to a total derivative. However, this direct method is very cumbersome and it is natural to seek for another more universal and easier approach. It will be developed in the next sections. As the important preparatory step, we note here that (5.26) admits the following equivalent representation through the symmetrized trace:

$$
\begin{aligned}
\mathcal{L}_{(1,1)}^{+4}= & \operatorname{Tr}_{(S)}\left\{\frac{1}{4} \varepsilon_{a b c d} W^{+a} W^{+b} W^{+c} W^{+d}+3 i q^{+A} \nabla_{a b} q_{A}^{+} W^{+a} W^{+b}-q^{+A} \nabla_{a b} q_{A}^{+} q^{+B} \nabla^{a b} q_{B}^{+}\right. \\
& \left.-W^{+a}\left[D_{a}^{+} q_{A}^{-}, q_{B}^{+}\right] q^{+A} q^{+B}-\frac{1}{2}\left[q^{+C}, q_{C}^{+}\right]\left[q_{A}^{-}, q_{B}^{+}\right] q^{+A} q^{+B}\right\} .
\end{aligned}
$$

The subscript $\mathrm{S}$ stands for the symmetrization, meaning that the expression is symmetrized with respect to the permutation of the four arguments,

$$
\begin{aligned}
& \operatorname{Tr}_{(S)}\left(A_{1} A_{2} A_{3} A_{4}\right) \\
& =\frac{1}{6} \operatorname{Tr}\left(A_{1} A_{2} A_{3} A_{4}+A_{2} A_{3} A_{1} A_{4}+A_{3} A_{1} A_{2} A_{4}+A_{3} A_{2} A_{1} A_{4}+A_{2} A_{1} A_{3} A_{4}+A_{1} A_{3} A_{2} A_{4}\right),
\end{aligned}
$$


any commutator being understood as one argument. One can now directly verify, in particular, that

$$
D_{a}^{+} \mathcal{L}_{(1,1)}^{+4}=\operatorname{Tr}_{(S)}\left\{E^{++}\left(\varepsilon_{a b c d} W^{+b} W^{+c} W^{+d}+6 i q^{+B} \nabla_{a b} q_{B}^{+} W^{+b}-\left[D_{a}^{+} q_{A}^{-}, q_{B}^{+}\right] q^{+A} q^{+B}\right)\right\}
$$

where $E^{++}=F^{++}+\frac{1}{2}\left[q^{+A}, q_{A}^{+}\right]=0$. This vanishes on mass shell.

Our final comment in this section is that the double-trace invariant (5.24) also admits an $\mathcal{N}=(1,1)$ completion. Here we present only the minimal $G$-analytic extension [analogous to the extension (5.25)]. It reads

$$
\begin{aligned}
\tilde{L}_{0}^{+4}= & \frac{1}{4} \varepsilon_{a b c d} \operatorname{Tr}\left(W^{+a} W^{+b}\right) \operatorname{Tr}\left(W^{+c} W^{+d}\right)-i \operatorname{Tr}\left(\nabla_{a b} q^{+A} q_{A}^{+}\right) \operatorname{Tr}\left(W^{+a} W^{+b}\right) \\
& +\operatorname{Tr}\left(D_{a}^{+} q^{-A} q_{A}^{+}\right) \operatorname{Tr}\left[W^{+a}\left(q^{+}\right)^{2}\right]-\operatorname{Tr}\left(q^{-A} q_{A}^{+}\right) \operatorname{Tr}\left[\left(q^{+}\right)^{2}\left(q^{+}\right)^{2}\right]
\end{aligned}
$$

It is straightforward to check that this expression is indeed annihilated by $D_{a}^{+}$on the mass shell. There exists a freedom of adding other on-shell analytic Lagrangians, like in the single-trace case. They all vanish in the limit of vanishing $q^{+A}$.

The double-trace analog of the on-shell $\mathcal{N}=(1,1)$ invariant $(5.26),(5.34)$ will be derived in the next sections, based on the universal method we are going to expose now.

\section{On-shell $\mathcal{N}=(1,1)$ harmonic superfields}

The most convenient way to construct on-shell $\mathcal{N}=(1,1)$ invariants of the type we discussed in the subsection 5.3 is to define the on-shell superfields living in extended harmonic $\mathcal{N}=(1,1)$ superspace. This and the next two sections are devoted to this subject. Extended on-shell superfields of the similar kind were first discussed in [10, 42], but not in the framework of harmonic superspace. We will see that "harmonization", introduced first in [11], helps a lot. In particular, it allowed us to resolve explicitly a set of constraints which the on-shell $\mathcal{N}=(1,1)$ SYM superfields must obey.

\subsection{The standard and harmonic $\mathcal{N}=(1,1)$ superspaces}

We introduce the extended superspace involving, in addition to the odd pseudoreal lefthanded variables $\theta_{i}^{a}$, also the odd pseudoreal right-handed variables $\hat{\theta}_{a}^{A}(A=1,2)$, which belong to another spinor representation,

$$
z=\left(x^{a b}, \theta_{i}^{a}\right) \Rightarrow \hat{z}=\left(x^{a b}, \theta_{i}^{a}, \hat{\theta}_{a}^{A}\right)
$$

We then consider the covariant spinor derivatives,

$$
\begin{aligned}
\nabla_{a}^{i} & =\frac{\partial}{\partial \theta_{i}^{a}}-i \theta^{b i} \partial_{a b}+\mathcal{A}_{a}^{i}, \\
\hat{\nabla}^{a A} & =\frac{\partial}{\partial \hat{\theta}_{A a}}-i \hat{\theta}_{b}^{A} \partial^{a b}+\hat{\mathcal{A}}^{a A},
\end{aligned}
$$


where $\mathcal{A}_{a}^{i}$ and $\hat{\mathcal{A}}^{a A}$ are the spinor connections and the convention $\nabla^{a b}=\frac{1}{2} \varepsilon^{a b c d} \nabla_{c d}$ is assumed. The superfields $\mathcal{A}_{a}^{i}, \hat{\mathcal{A}}^{a A}$ are not arbitrary, but satisfy the constraints

$$
\begin{aligned}
& \left\{\nabla_{a}^{(i}, \nabla_{b}^{j)}\right\}=\left\{\hat{\nabla}^{a(A}, \hat{\nabla}^{b B)}\right\}=0, \\
& \left\{\nabla_{a}^{i}, \hat{\nabla}^{b A}\right\}=\delta_{a}^{b} \phi^{i A} .
\end{aligned}
$$

Bearing in mind the Bianchi identities, the constraints (6.3) and (6.4) imply

$$
\nabla_{a}^{(i} \phi^{j) A}=\hat{\nabla}^{a(A} \phi^{B) i}=0 .
$$

The constraints (6.3), (6.4), written in [10,42], define the $\mathcal{N}=(1,1), 6 D$ supersymmetric Yang-Mills theory. They are known to imply the equations of motion for the superfields involved. Below we will show how this property comes about in the harmonic superfield formalism.

We introduce now the harmonics $u_{A}^{\hat{ \pm}}$ which parametrize the second $\mathrm{SU}(2)$ automorphism group acting on the indices $A$ and have the same properties as $u_{i}^{ \pm}$. Note, in particular, the identities

$$
u^{\hat{+} A} u_{A}^{\hat{\bar{A}}}=1, \quad u_{A}^{\hat{+}} u_{B}^{\hat{-}}-u_{A}^{\hat{\bar{A}}} u_{B}^{\hat{+}}=\epsilon_{A B} .
$$

Respectively, we extend the $\mathcal{N}=(1,0)$ harmonic superspace $(3.12)$ to the $\mathcal{N}=(1,1)$ harmonic superspace

$$
Z=\left(x^{a b}, \theta_{i}^{a}, u_{k}^{ \pm}\right) \Rightarrow \hat{Z}=\left(x^{a b}, \theta_{i}^{a}, \hat{\theta}_{a}^{A}, u_{k}^{ \pm}, u_{A}^{\hat{ \pm}}\right) .
$$

The analytic basis of this extended harmonic superspace is defined as the set of coordinates

$$
\hat{Z}_{(\mathrm{an})}=\left(x_{(\mathrm{an})}^{a b}, \theta^{ \pm a}, \theta_{a}^{\hat{ \pm}}, u_{k}^{ \pm}, u_{A}^{\hat{ \pm}}\right)
$$

where $\theta_{a}^{\hat{ \pm}}:=\hat{\theta}_{a}^{A} u_{A}^{\hat{x}}$ and

$$
x_{(\mathrm{an})}^{a b}=x^{a b}+\frac{i}{2}\left(\theta^{+a} \theta^{-b}-\theta^{+b} \theta^{-a}\right)+\frac{i}{2} \varepsilon^{a b c d} \theta_{c}^{\hat{+}} \theta_{d}^{\hat{-}} .
$$

Next we define the harmonic projection $\phi^{+\hat{+}}=\phi^{i A} u_{i}^{+} u_{A}^{\hat{+}}$. It is clear from (6.5) and from the fact that $\phi^{+\hat{+}}$ does not depend on $u_{i}^{-}$and $u_{A}^{\hat{-}}$ that $\phi^{+\hat{+}}$ satisfies the constraints

$$
\nabla_{a}^{+} \phi^{+\hat{+}}=\nabla^{a \hat{+}} \phi^{+\hat{+}}=\partial^{++} \phi^{+\hat{+}}=\partial^{\hat{+} \hat{+}} \phi^{+\hat{+}}=0
$$

where

$$
\nabla_{a}^{+}=\nabla_{a}^{i} u_{i}^{+}, \quad \nabla^{\hat{+} a}=\hat{\nabla}^{a A} u_{A}^{\hat{+}}, \quad \partial^{++}=u^{+i} \frac{\partial}{\partial u^{-i}}, \quad \partial^{\hat{+} \hat{+}}=u^{\hat{+} A} \frac{\partial}{\partial u^{\hat{-A}}} .
$$

The spinor covariant derivatives obviously commute with $\partial^{++}$and $\partial^{\hat{+}} \hat{+}$. The full set of defining (anti)commutators of the gauge $\mathcal{N}=(1,1), 6 D$ theory in the central basis of the considered bi-harmonic superspace are

$$
\begin{aligned}
\left\{\nabla_{a}^{+}, \nabla_{b}^{+}\right\} & =\left\{\nabla^{\hat{+} a}, \nabla^{\hat{+} b}\right\}=0, \\
\left\{\nabla_{a}^{+}, \nabla^{\hat{+} b}\right\} & =\delta_{a}^{b} \phi^{+\hat{+}}, \\
{\left[\partial^{++}, \nabla_{a}^{+}\right] } & =\left[\partial^{\hat{+} \hat{+}}, \nabla_{a}^{+}\right]=\left[\partial^{++}, \nabla^{a \hat{+}}\right]=\left[\partial^{\hat{+} \hat{+}}, \nabla^{a \hat{+}}\right]=0 .
\end{aligned}
$$


Note that, having defined this set, we do not longer need to assume in advance that the + and $\hat{+}$ components of the spinor derivatives are as in (6.11). It is the relations (6.14) which force them to be linear in harmonics. Thus, the extended set of constraints (6.12)(6.14) is fully equivalent to the original constraints (6.3), (6.4) without any additional assumptions. The constraints (6.10) naturally arise as a consequence of Bianchi identities for $(6.12)-(6.14)$.

\subsection{From the central basis to the analytic basis}

As usual in the harmonic superspace approach, at the next steps we should pass to the analytic basis in order to solve the above constraints in terms of the appropriate analytic superfield prepotentials and, in particular, to find the explicit form of the basic superfield strength $\phi^{+\hat{+}}$. Due to the relation (6.13), the analyticities associated with the harmonic sets $u_{i}^{ \pm}$and $u_{A}^{\hat{x}}$ cannot be made manifest simultaneously. In what follows, we will choose the basis in which the spinor derivative $\nabla^{\hat{+} a}$ is short, $\nabla^{\hat{+} a}=\partial / \partial \theta_{a}^{\hat{-}}$, so that the "hat" analyticity is manifest.

Consider first the abelian case, which is much simpler. Our task is to find a field $\phi^{+\hat{+}}$ that satisfies the constraints (6.10). In the abelian case, the field $\phi^{\hat{+}+}$ does not carry a charge with respect to the gauge $\mathrm{U}(1)$ group, and, as a result, the constraints $\nabla_{a}^{+} \phi^{\hat{+}+}=\nabla^{\hat{+} a} \phi^{\hat{+}+}=0$ amount to $D_{a}^{+} \phi^{\hat{+}+}=D^{\hat{+} a} \phi^{\hat{+}+}=0$ with flat spinor derivatives. The anticommutator $\left\{D_{a}^{+}, D^{\hat{+} b}\right\}$ vanishes, so these derivatives can be made "short" by passing to the double analytic basis, where $D_{a}^{+}=\partial / \partial \theta^{-a}$ and $D_{a}^{\hat{+}}=\partial / \partial \theta_{a}^{\hat{-}}$. On the contrary, the harmonic derivatives in this basis are lengthened:

$$
\begin{aligned}
& D^{++}=\partial^{++}+i \theta^{+a} \theta^{+b} \partial_{a b}^{(\mathrm{an})}+\theta^{+a} \frac{\partial}{\partial \theta^{-a}} \\
& D^{\hat{+} \hat{+}}=\partial^{\hat{+} \hat{+}}+i \theta_{a}^{\hat{+}} \theta_{b}^{\hat{+}} \partial^{a b(\mathrm{an})}+\theta_{a}^{\hat{+}} \frac{\partial}{\partial \theta_{a}^{\hat{-}}}
\end{aligned}
$$

It is not difficult now to resolve the abelian constraints (6.10). The solution reads

$$
\begin{aligned}
\phi^{+\hat{+}}= & \varphi^{+\hat{+}}-\theta^{+a} \psi_{a}^{\hat{+}}-\theta_{a}^{\hat{+}} \lambda^{+a}+\frac{i}{6} \theta_{a}^{\hat{+}} \theta^{+b} F_{b}^{a}-i \theta^{+a} \theta^{+b} \partial_{a b} \varphi^{-\hat{+}} \\
& -i \theta_{a}^{\hat{+}} \theta_{b}^{\hat{+}} \partial^{a b} \varphi^{+\hat{-}}+i \theta_{a}^{\hat{+}} \theta^{+b} \theta^{+c} \partial_{b c} \lambda^{-a}+i \theta^{+a} \theta_{b}^{\hat{+}} \theta_{c}^{\hat{+}} \partial^{b c} \psi_{a}^{\hat{-}} \\
& -\theta_{a}^{\hat{+}} \theta_{b}^{\hat{+}} \theta^{+c} \theta^{+d} \partial^{a b} \partial_{c d} \varphi^{-\hat{-}} .
\end{aligned}
$$

Here, the fermionic fields satisfy the Dirac equations $\partial_{a b} \lambda^{a}=\partial^{a b} \psi_{a}=0$, the scalar field satisfies $\square \varphi=0$ and $F_{b}^{a}=\left(\sigma^{M N}\right)_{b}^{a} F_{M N}$. We see that the superfield $\phi^{+\hat{+}}$ satisfying our constraints automatically satisfies also the equations of motion, i.e. it is an on-shell superfield.

For sure, this should not come as a surprise. The same is true for the free hypermultiplet superfield $q^{+}$in the usual $\mathcal{N}=(1,0)$ superspace. In the abelian case, this superfield satisfies the constraints $D^{+} q^{+}=D^{++} q^{+}=0$. Its component expansion in the analytic basis is given by (4.13), with scalar and fermionic fields satisfying the free equations of 
motion. The component expansion (6.16) of the free superfield $\phi^{+\hat{+}}$ represents an obvious generalization of (4.13). ${ }^{12}$

Now we come back to the general non-abelian case. Consider the constraint $\left\{\nabla^{\hat{+} a}, \nabla^{\hat{+} b}\right\}=0$. Its generic solution is

$$
\nabla^{\hat{+} a}=e^{i V} D^{\hat{+} a} e^{-i V}
$$

where $V$ is a general bi-harmonic superfield (often called bridge). It is convenient now to perform the similarity transformation

$$
\nabla^{\hat{+} a} \rightarrow D^{\hat{+} a}, \quad \phi^{+\hat{+}} \rightarrow e^{-i V} \phi^{+\hat{+}} e^{i V}, \quad \nabla_{a}^{+} \rightarrow e^{-i V} \nabla_{a}^{+} e^{i V}
$$

and define

$$
\begin{array}{rlrl}
\nabla^{++} & =e^{-i V} \partial^{++} e^{i V}=\partial^{++}+V^{++}, & \nabla^{\hat{+} \hat{+}} & =e^{-i V} \partial^{\hat{+} \hat{+}} e^{i V}=\partial^{\hat{+} \hat{+}}+V^{\hat{+} \hat{+}}, \\
V^{++} & :=e^{-i V}\left(\partial^{++} e^{i V}\right), & V^{\hat{+} \hat{+}}:=e^{-i V}\left(\partial^{\hat{+} \hat{+}} e^{i V}\right) .
\end{array}
$$

The transformed spinor derivatives still satisfy the algebra (6.12)-(6.14) and commute with the transformed harmonic derivatives (which involve now nontrivial harmonic connections $V^{++}$and $V^{\hat{+} \hat{+}}$ ). As was anticipated, to resolve the constraints, we go to the "hat-analytic" basis, ${ }^{13}$ where $D^{\hat{+} a}=\partial / \partial \theta^{\hat{-a}}$ and $\partial^{\hat{+} \hat{+}}$ goes over to $D^{\hat{+} \hat{+}}$ defined in (6.15).

In the next section we will solve the system of constraints

$$
\nabla_{a}^{+} \phi^{+\hat{+}}=D^{a \hat{+}} \phi^{+\hat{+}}=\nabla^{++} \phi^{+\hat{+}}=\nabla^{\hat{+} \hat{+}} \phi^{+\hat{+}}=0
$$

with the spinor and harmonic covariant derivatives given in the analytic basis and frame and satisfying the algebra

$$
\begin{aligned}
\left\{\nabla_{a}^{+}, \nabla_{b}^{+}\right\} & =\left\{D^{\hat{+} a}, D^{\hat{+} b}\right\}=0, \\
\left\{\nabla_{a}^{+}, D^{\hat{+} b}\right\} & =\delta_{a}^{b} \phi^{+\hat{+}}, \\
{\left[\nabla^{++}, \nabla_{a}^{+}\right] } & =\left[\nabla^{\hat{+}}, \nabla_{a}^{+}\right]=\left[\nabla^{++}, D^{a \hat{+}}\right]=\left[\nabla^{\hat{+} \hat{+}}, D^{a \hat{+}}\right]=0, \\
{\left[\nabla^{++}, \nabla^{\hat{+}}\right] } & =0,
\end{aligned}
$$

which directly follows from the constraints (6.12)-(6.14) written in the central basis.

One can now verify that an explicit solution of the system of equations (6.22)-(6.25) is

$$
\begin{aligned}
\nabla_{a}^{+} & =D_{a}^{+}-\theta_{a}^{\hat{+}} q^{+\hat{-}}+\theta_{a}^{\hat{-}} \phi^{+\hat{+}} \\
V^{\hat{+}} \hat{+} & =i \theta_{a}^{\hat{+}} \theta_{b}^{\hat{+}} \mathcal{A}^{a b}-\frac{1}{3} \epsilon^{a b c d} \theta_{a}^{\hat{+}} \theta_{b}^{\hat{+}} \theta_{c}^{\hat{+}} D_{d}^{+} q^{-\hat{-}}+\frac{1}{8} \epsilon^{a b c d} \theta_{a}^{\hat{+}} \theta_{b}^{\hat{+}} \theta_{c}^{\hat{+}} \theta_{d}^{\hat{+}}\left[q^{+\hat{-}}, q^{-\hat{-}}\right]
\end{aligned}
$$

\footnotetext{
${ }^{12}$ It is also possible to define the off-shell harmonic $\mathcal{N}=(1,0)$ superfield $q^{+}$whose expansion into harmonics gives an infinite number of degrees of freedom. For the superfield $\phi^{+\hat{+}}$, this seems to be impossible.

${ }^{13} \mathrm{By}$ performing the similarity transformation and going to this basis, we can get rid only of one of the spinor connections, which we have chosen to be $\mathcal{A}^{\hat{+} a}$. Alternatively, one could suppress $\mathcal{A}_{i}^{+}$.
} 
and

$$
\begin{aligned}
\phi^{+\hat{+}}= & q^{+\hat{+}}-\theta_{a}^{\hat{+}} W^{+a}-i \theta_{a}^{\hat{+}} \theta_{b}^{\hat{+}} \nabla^{a b} q^{+\hat{-}}+\frac{1}{6} \varepsilon^{a b c d} \theta_{a}^{\hat{+}} \theta_{b}^{\hat{+}} \theta_{c}^{\hat{+}}\left[D_{d}^{+} q^{-\hat{-}}, q^{+\hat{-}}\right] \\
& +\frac{1}{24} \varepsilon^{a b c d} \theta_{a}^{\hat{+}} \theta_{b}^{\hat{+}} \theta_{c}^{\hat{+}} \theta_{d}^{\hat{+}}\left[q^{+\hat{-}},\left[q^{+\hat{-}}, q^{-\hat{-}}\right]\right] .
\end{aligned}
$$

Here the objects $q^{+\hat{ \pm}}=q^{+A} u_{A}^{\hat{x}}$ and $q^{+A}, \mathcal{A}^{a b}, W^{+a}$, as well as $V^{++}$entering the covariant derivative $\nabla^{++}$, are the $\mathcal{N}=(1,0)$ superfields discussed in the previous sections. For self-consistency, they should satisfy their equations of motion, e.g., $\nabla^{++} q^{+A}=0$. In the next section, we will present an accurate derivation of this solution from the set of constraints (6.22)-(6.25) and show thereby that the solution (6.26)-(6.28) is unique. We will also derive the variations of the on-shell superfields $\phi^{+\hat{+}}$ and $V^{\hat{+} \hat{+}}$ under the $\mathcal{N}=(0,1)$ supersymmetry transformations and demonstrate that the particular representation (6.27) for the gauge superfield $V^{\hat{+} \hat{+}}$ in (6.27) is none other than the appropriate Wess-Zumino gauge choice for it.

In the abelian case, the commutators vanish, the covariant derivative $\nabla^{a b}$ is replaced by the ordinary one, and the superfield (6.28) is reduced to the abelian superfield (6.16) ( $\lambda^{a}$ being the lowest component of $W^{+a}$ ). Note that the non-abelian expression for $\phi^{+\hat{+}}$ does not enjoy anymore the symmetry under interchange $\theta \leftrightarrow \hat{\theta}$. That is due to our choice to work in the frame, where the hatted spin connection vanishes.

To close this section, we write the variations of the superfields (6.27), (6.28) under the $\mathcal{N}=(0,1)$ supertransformations, just anticipating their derivation in the next section:

$$
\begin{aligned}
\delta V^{\hat{+} \hat{+}} & =-\epsilon_{a}^{\hat{+}} \frac{\partial}{\partial \theta_{a}^{\hat{+}}} V^{\hat{+} \hat{+}}-2 i \epsilon_{a}^{\hat{-}} \theta_{b}^{\hat{+}} \partial^{a b} \phi^{+\hat{+}}+\nabla^{\hat{+} \hat{+}} \Lambda^{(\mathrm{comp})}, \\
\delta \phi^{+\hat{+}} & =-\epsilon_{a}^{\hat{+}} \frac{\partial}{\partial \theta_{a}^{\hat{+}}} \phi^{+\hat{+}}-2 i \epsilon_{a}^{\hat{-}} \theta_{b}^{\hat{+}} \partial^{a b} \phi^{+\hat{+}}-\left[\Lambda^{(\mathrm{comp})}, \phi^{+\hat{+}}\right],
\end{aligned}
$$

where the field-dependent compensating gauge parameter $\Lambda^{(\mathrm{comp})}$ is given by the expression

$$
\begin{aligned}
\Lambda^{(\mathrm{comp})}= & \left(\epsilon^{-B} q_{B}^{+}\right)+2 i \epsilon_{a}^{\hat{-}} \theta_{b}^{\hat{+}} \mathcal{A}^{a b}-\frac{1}{2} \varepsilon^{a b c d} \hat{\epsilon_{a}^{-}} \theta_{b}^{\hat{+}} \theta_{c}^{\hat{+}} D_{d}^{+} q^{-\hat{-}} \\
& +\frac{1}{6} \varepsilon^{a b c d} \epsilon_{a}^{\hat{-}} \theta_{b}^{\hat{+}} \theta_{c}^{\hat{+}} \theta_{d}^{\hat{+}}\left[q^{+\hat{-}}, q^{-\hat{-}}\right] .
\end{aligned}
$$

The first two terms in (6.30) and (6.29) are induced by the supersymmetric variations of $\theta_{a}^{\hat{+}}$ and $x^{a b}$. The third term is an extra gauge transformation needed to preserve the Wess-Zumino form of the superfield $V^{\hat{+} \hat{+}}$ after the supersymmetry transformation. It is worth pointing out that the simple form (6.30) and (6.29) of the hidden $\mathcal{N}=(0,1)$ transformations is obtained, provided that the involved $\mathcal{N}=(1,0)$ superfields are subject to their equations of motion. At the same time, under the manifest $\mathcal{N}=(1,0)$ supersymmetry the expressions (6.28) and (6.27) behave as the standard off-shell $\mathcal{N}=(1,0)$ harmonic superfields.

In fact, the transformations (6.30) and (6.29) can be derived directly from the on-shell transformation laws (4.28)-(4.31) of the involved $\mathcal{N}=(1,0)$ superfields, using the identities listed in appendix A. 


\section{Solving the $\mathcal{N}=(1,1)$ SYM constraints in terms of $\mathcal{N}=(1,0)$ super- fields}

In this section, we solve the constraints in the analytic basis and frame and show that their general solution is given by eqs. (6.26), (6.27), (6.28).

\subsection{Input and gauge-fixing}

We start with the whole set of constraints (6.12)-(6.14) written in a more detailed form,

$$
\begin{aligned}
\text { (a) }\left\{\nabla_{a}^{+}, \nabla_{b}^{+}\right\} & =0, \text { (b) }\left\{D^{\hat{+} a}, D^{\hat{+} b}\right\}=0, \quad \text { (c) }\left\{\nabla_{a}^{+}, D^{\hat{+} b}\right\}=\delta_{a}^{b} \phi^{+\hat{+}} \\
\text { (a) }\left[\nabla^{\hat{+}} \hat{+}, \nabla_{a}^{+}\right] & =0, \quad \text { (b) }\left[\tilde{\nabla}^{++}, \nabla_{a}^{+}\right]=0, \text { (c) }\left[\nabla^{\hat{+} \hat{+}}, D^{a \hat{+}}\right]=0, \quad \text { (d) }\left[\tilde{\nabla}^{++}, D^{a \hat{+}}\right]=0, \\
{\left[\tilde{\nabla}^{++}, \nabla^{\hat{+} \hat{+}}\right] } & =0 .
\end{aligned}
$$

Here

$$
\nabla_{a}^{+}=D_{a}^{+}+\mathcal{A}_{a}^{+}(\hat{Z})
$$

and the "hatted" spinor derivatives were chosen to be short, $\nabla^{\hat{+} a}=D^{\hat{+} a}=\partial / \partial \theta_{a}^{\hat{-}} \cdot{ }^{14}$ Thus, in the chosen basis, the "hatted" G-analyticity is manifest ${ }^{15}$ and the constraints (7.2c) and (7.2d) imply that both harmonic gauge connections in the harmonic derivatives $\tilde{\nabla}^{++}$ and $\nabla^{\hat{+}} \hat{+}$ are independent of the coordinates $\theta_{b}^{\hat{-}}$ :

$$
\tilde{\nabla}^{++}=D^{++}+\tilde{V}^{++}(\hat{\zeta}), \quad \nabla^{\hat{+} \hat{+}}=D^{\hat{+} \hat{+}}+V^{\hat{+} \hat{+}}(\hat{\zeta})
$$

where $\hat{\zeta}=\left(x_{(\text {an })}^{a b}, \theta^{ \pm a}, \theta_{a}^{\hat{+}}, u_{i}^{ \pm}, u_{A}^{\hat{ \pm}}\right)$. In what follows, we omit the index "(an)" for the analytic coordinate $x$. We use the notation $\tilde{\nabla}^{++}$in order to distinguish this harmonic derivative acting in the full $\mathcal{N}=(1,1)$ superspace from its $\mathcal{N}=(1,0)$ counterpart.

At this step, both harmonic connections are arbitrary functions of the hatted analytic coordinates $\theta_{a}^{\hat{+}}$ and the harmonics $u_{A}^{\hat{x}}$, as well as of the full set of the $\mathcal{N}=(1,0)$ harmonic superspace coordinates. They are transformed with the hat-analytic superfield parameter $\Lambda(\tilde{\zeta})$ :

$$
\begin{aligned}
& \delta \tilde{V}^{++}=\tilde{\nabla}^{++} \Lambda(\hat{\zeta}), \\
& \delta V^{\hat{+} \hat{+}}=\nabla^{\hat{+} \hat{+}} \Lambda(\hat{\zeta}) .
\end{aligned}
$$

The constraints (7.2c) and (7.2d) imply no other consequences.

As the next steps, we wish to show that the dependence of the harmonic connections $V^{\hat{+} \hat{+}}$ and $\tilde{V}^{++}$on the coordinates $\theta_{a}^{\hat{+}}, u_{A}^{\hat{x}}$ can be drastically simplified (i) by choosing the Wess-Zumino- type gauge for $V^{\hat{+} \hat{+}}$ and (ii) by exploiting the constraint (7.3) for $\tilde{V}^{++}$(see the next subsection).

\footnotetext{
${ }^{14}$ One can always get rid of the spinor connection $\hat{\mathcal{A}}$ in the covariant derivatives $\nabla^{\hat{+} a}$, capitalizing on their anticommutativity in any basis and frame.

${ }^{15}$ In the general non-abelian case, one cannot make simultaneously manifest both the hatted and unhatted G-analyticities because of the non-vanishing anticommutator (7.1c).
} 
It is straightforward to see that the gauge freedom associated with the superfield transformation parameter $\Lambda(\hat{\zeta})$ can be partially fixed by putting $V^{\hat{+} \hat{+}}$ in the "short" form, ${ }^{16}$

$$
V^{\hat{+} \hat{+}}=i \theta_{a}^{\hat{+}} \theta_{b}^{\hat{+}} \hat{\mathcal{A}}^{a b}+\Psi^{\hat{+} 3 d} \varphi_{d}^{\hat{\bar{d}}}+\Psi^{\hat{+} 4} \mathcal{D}^{\hat{-} 2}, \quad \varphi_{d}^{\hat{\bar{c}}}=\varphi_{d}^{A} u_{\bar{A}}^{\hat{\bar{A}}}, \quad \mathcal{D}^{\hat{-} 2}=\mathcal{D}^{(A B)} u_{\bar{A}}^{\hat{\bar{c}}},
$$

where $\hat{\mathcal{A}}^{a b}, \varphi_{d}^{A}$ and $\mathcal{D}^{(A B)}$ are some $\mathcal{N}=(1,0)$ harmonic superfields, still arbitrary at this step. While passing to $(7.8)$, the $\left(\theta_{a}^{\hat{+}}, u_{A}^{\hat{ \pm}}\right)$ dependence of $\Lambda(\hat{\zeta})$ was fully used up, so the residual gauge freedom is associated with the gauge function $\Lambda_{\text {int }}\left(x, u_{i}^{ \pm}, \theta^{ \pm a}\right), \Lambda \rightarrow \Lambda_{\text {int }}$. Note that this gauge parameter still depends on $\theta^{-a}$. Now we are going to show that this dependence can be fixed by a further gauge choice.

To this end, we need to inspect the structure of the spinor derivative $\nabla_{a}^{+}=D_{a}^{+}+\mathcal{A}_{a}^{+}$. First of all, the Bianchi identities, following from the full set (7.1), imply the G-analyticity conditions for $\phi^{+\hat{+}}$,

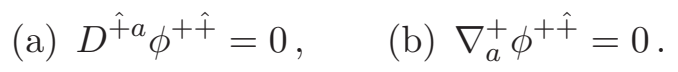

Postponing the discussion of the condition $(7.9 \mathrm{~b})$ to the next subsection, we focus here on the constraint (7.9a). Due to the "shortness" of $D^{\hat{+} a}$, it implies that $\phi^{+\hat{+}}$ does not depend on $\theta_{a}^{\hat{-}}$. In addition, this constraint together with (7.1c) uniquely fixes the spinor connection $\mathcal{A}_{a}^{+}$to be

$$
\mathcal{A}_{a}^{+}=\tilde{\mathcal{A}}_{a}^{+}+\theta_{a}^{-} \phi^{+\hat{+}}
$$

where

$$
\tilde{\mathcal{A}}_{a}^{+}=f_{a}^{+}+\theta_{b}^{\hat{+}} f_{a}^{+\hat{-} b}+\theta_{b}^{\hat{+}} \theta_{c}^{\hat{+}} f_{a}^{+\hat{\mathcal{A}} b c}+\Psi^{\hat{+} 3 d} f_{d}^{+\hat{-} 3}+\Psi^{\hat{+} 4} f_{a}^{+\hat{\mathcal{-}} 4} .
$$

The component superfields in this expansion depend on both the $\mathcal{N}=(1,0)$ coordinates (including the harmonics $u_{i}^{ \pm}$) and the extra harmonics $u_{A}^{\hat{ \pm}}$.

One of the consequences of the constraint (7.1a) is

$$
D_{a}^{+} f_{b}^{+}+D_{b}^{+} f_{a}^{+}+\left\{f_{a}^{+}, f_{b}^{+}\right\}=0,
$$

whence $f_{b}^{+}=e^{i \tilde{v}}\left(D_{b}^{+} e^{-i \tilde{v}}\right)$, where $\tilde{v}$ is an additional "bridge" which does not depend on $\theta_{a}^{\hat{+}}$ (because $f_{b}^{+}$does not). Using this bridge, we can pass to the frame where $f_{b}^{+}=0$ and the residual gauge group is represented by the standard analytic superfield parameter $\Lambda(\zeta)$ of the $\mathcal{N}=(1,0)$ gauge theory. Indeed, the residual gauge transformations preserving the condition $f_{b}^{+}=0$ commute with $D_{a}^{+}$, whence $D_{a}^{+} \Lambda=0$.

Hereafter, we will use the spinor connection $\mathcal{A}_{a}^{+}$in the form (7.10), (7.11) with the condition

$$
f_{a}^{+}=0,
$$

and the following $\theta_{b}^{+}$expansions for the hat-analytic superfields $\phi^{+\hat{+}}$ and $\tilde{V}^{++}$,

$$
\begin{aligned}
\phi^{+\hat{+}} & =q^{+\hat{+}}-\theta_{a}^{\hat{+}} W^{+a}+\theta_{a}^{\hat{+}} \theta_{b}^{\hat{+}} \beta^{+\hat{-} a b}+\Psi^{\hat{+} 3 d} G_{d}^{+\hat{-}} \hat{-}+\Psi^{\hat{+} 4} G^{+\hat{-} 3}, \\
\tilde{V}^{++} & =V^{++}+\theta_{a}^{\hat{+}} v^{++\hat{-} a}+\theta_{a}^{\hat{+}} \theta_{b}^{\hat{+}} v^{++\hat{-}} a b+\Psi^{\hat{+} 3 d} v_{d}^{++\hat{-} 3}+\Psi^{\hat{+} 4} v^{++\hat{-} 4} .
\end{aligned}
$$

\footnotetext{
${ }^{16}$ For further convenience, we use the abbreviations $\Psi^{\hat{+} 3 d}:=\varepsilon^{a b c d} \theta_{a}^{\hat{+}} \theta_{b}^{\hat{+}} \theta_{c}^{\hat{+}}, \Psi^{\hat{+} 4}:=\varepsilon^{a b c d} \theta_{a}^{\hat{+}} \theta_{b}^{\hat{+}} \theta_{c}^{\hat{+}} \theta_{d}^{\hat{+}}$. The identities $\theta_{a}^{\hat{+}} \theta_{b}^{\hat{+}} \theta_{c}^{\hat{+}}=\frac{1}{6} \varepsilon_{a b c d} \Psi^{\hat{+} 3 d}, \quad \theta_{a}^{\hat{+}} \theta_{b}^{\hat{+}} \theta_{c}^{\hat{+}} \theta_{d}^{\hat{+}}=\frac{1}{24} \varepsilon_{a b c d} \Psi^{\hat{+} 4}, \quad \theta_{a}^{\hat{+}} \Psi^{\hat{+} 3 b}=-\frac{1}{4} \delta_{a}^{b} \Psi^{\hat{+} 4}$ hold.
} 
In (7.14), (7.15) we introduced the notation $q^{+\hat{+}}, W^{+a}$ and $V^{++}$, having in mind that these quantities will be finally identified with the $\mathcal{N}=(1,0)$ superfields considered before. However, at the present stage, all the coefficients in the expansions (7.14), (7.15) are still generic $\mathcal{N}=(1,0)$ superfields involving an extra dependence on the harmonics $u_{A}^{\hat{f}}$.

Now we are ready to explore all the consequences of the constraints (7.1)-(7.3).

\subsection{Harmonic equations}

We start by showing that $\tilde{V}^{++}$does not actually depend on the coordinates $\theta_{a}^{\hat{+}}$ and $u_{A}^{\hat{f}}$, if fixing the gauge as in (7.8). This follows from the constraint (7.3), which amounts to the mixed "harmonic flatness" condition

$$
D^{++} V^{\hat{+} \hat{+}}-D^{\hat{+} \hat{+}} \tilde{V}^{++}+\left[\tilde{V}^{++}, V^{\hat{+} \hat{+}}\right]=0 .
$$

Substituting the WZ expression (7.8) for $V^{\hat{+} \hat{+}}$ and equating to zero the coefficients in the $\theta_{a}^{-}$expansion of the 1.h.s. of (7.16), we find the set of equations

$$
\begin{aligned}
& \partial^{\hat{+} \hat{+}} \tilde{V}^{++}=0, \quad \partial^{\hat{+} \hat{+}} v^{++\hat{-} a}=0, \\
& \partial^{\hat{+} \hat{+}} v^{++\hat{-} \hat{-} a b}-i\left(\nabla^{++} \hat{A} a b-\partial^{a b} \tilde{V}^{++}\right)=0, \\
& \partial^{\hat{+} \hat{+}} v_{d}^{++\hat{-} 3}-\nabla^{++} \varphi_{d}^{A} u_{A}^{\hat{-}}=0, \\
& \partial^{\hat{+} \hat{+}} v^{++\hat{-} 4}-\nabla^{++} \mathcal{D}^{A B} u_{A}^{\hat{-}} u_{B}^{-}=0 .
\end{aligned}
$$

Eqs. (7.17) imply the independence of $V^{++}$of the harmonics $u_{A}^{\hat{x}}$ and, bearing in mind the Lemma (3.43), also the condition

$$
v^{++\hat{-} a}=0 .
$$

Already at this step we can identify $\tilde{V}^{++}$with the familiar from the previous sections harmonic $\mathcal{N}=(1,0)$ gauge potential, since the $\theta_{a}^{\hat{x}}$ - independent part of the constraint $(7.2 \mathrm{~b})$ is equivalent to the $\mathcal{N}=(1,0)$ G-analyticity condition, $(7.2 \mathrm{~b}) \rightarrow D_{a}^{+} \tilde{V}^{++}=0$.

Eq. (7.18) is equivalent to two separate equations, the one for $v^{++\hat{-}} \hat{-a b}$, which implies

$$
v^{++\hat{-} \hat{-} a b}=0,
$$

and another independent condition arising in the zero order in $u^{\hat{ \pm}}$,

$$
\nabla^{++} \hat{A}^{a b}-\partial^{a b} \tilde{V}^{++}=0 .
$$

Analogously, the remaining equations (7.19) and (7.20) imply

$$
v_{d}^{++\hat{-} 3}=v^{++\hat{-} 4}=0,
$$

as well as

$$
\nabla^{++} \varphi_{d}^{A}=0, \quad \nabla^{++} \mathcal{D}^{A B}=0
$$

Thus, we derived that

$$
\tilde{V}^{++} \equiv V^{++}, \quad \tilde{\nabla}^{++} \equiv \nabla^{++} .
$$


We have also obtained the harmonic constraints (7.23) and (7.25). Note that (7.23) is equivalent to the vanishing of the commutator

$$
\left[\nabla^{++}, \hat{\nabla}^{a b}\right]=0, \quad \hat{\nabla}^{a b}:=\partial^{a b}+\hat{A}^{a b} .
$$

The constraint (7.3) has thereby been fully used and solved.

Our next task is to further fix the spinor connection (7.10). It involves the superfield $\phi^{+\hat{+}}$. Consider it in more details. Besides the G-analyticity conditions (7.9), it satisfies the harmonic constraints

$$
\text { (a) } \nabla^{\hat{+} \hat{+}} \phi^{+\hat{+}}=0 ; \quad \text { (b) } \nabla^{++} \phi^{+\hat{+}}=0
$$

which also come out as the Bianchi identities [they are derived by commuting both sides of (7.1c) with $\nabla^{\hat{+} \hat{+}}$ and $\tilde{\nabla}^{++}=\nabla^{++}$and taking into account the constraints (7.2)]. Eq. (7.28a) amounts to the following set of equations for the $\mathcal{N}=(1,0)$ components in the expansion (7.14):

$$
\begin{gathered}
\partial^{\hat{+} \hat{+}} q^{+\hat{+}}=0 \Rightarrow q^{+\hat{+}}=q^{+A} u_{A}^{\hat{+}}, \\
\partial^{\hat{+} \hat{+}} \beta^{+\hat{-} a b}+i \hat{\nabla}^{a b} q^{+\hat{+}}=0 \Rightarrow \beta^{+\hat{-} a b}=-i \hat{\nabla}^{a b} q^{+\hat{-}}, \quad q^{+\hat{-}}:=q^{+A} u_{A}^{\hat{\bar{A}}}, \\
\partial^{\hat{+} \hat{+}} G_{d}^{+\hat{-} 2}+\left[\varphi_{d}^{\hat{-}}, q^{+\hat{+}}\right]+\frac{i}{6} \varepsilon_{d a b c} \hat{\nabla}^{a b} W^{+c}=0, \\
\partial^{\hat{+} \hat{+}} G^{+\hat{-} 3}+\frac{1}{24} \varepsilon_{a b c d} \hat{\nabla}^{a b} \hat{\nabla}^{c d} q^{+\hat{+}}+\left[\mathcal{D}^{\hat{-} 2}, q^{+\hat{+}}\right]+\frac{1}{4}\left\{\varphi_{a}^{\hat{-}}, W^{+a}\right\}=0,
\end{gathered}
$$

where $\hat{\nabla}^{a b}=\partial^{a b}+\hat{\mathcal{A}}^{a b}$. Eqs. (7.31) and (7.32) amount to the equations for defining the superfields $G_{d}^{+\hat{-} 2}, G^{+\hat{-} 3}$ and to the additional self-consistency conditions which appear in the zero order in harmonics $u_{A}^{\hat{x}}$,

$$
\begin{aligned}
\varepsilon_{d a b c} \hat{\nabla}^{a b} W^{+c}-3 i\left[\varphi_{d A}, q^{+A}\right] & =0 \\
\varepsilon_{a b c d} \hat{\nabla}^{a b} \hat{\nabla}^{c d} q^{+A}+6\left\{\varphi_{a}^{A}, W^{+a}\right\}-16\left[\mathcal{D}^{A B}, q_{B}^{+}\right] & =0 .
\end{aligned}
$$

These self-consistency conditions can be shown to be satisfied on the final solution of the constraints. The harmonic equations for $G_{d}^{+\hat{-} 2}$ and $G^{+\hat{-} 3}$ uniquely fix these superfields as

$$
\begin{aligned}
G_{d}^{+\hat{-} 2} & =G_{d}^{+(A B)} u_{A}^{\hat{\bar{A}}} u_{\bar{B}}^{\hat{\bar{B}}}, & G_{d}^{+(A B)} & =-\frac{1}{2}\left[\varphi_{d}^{(A}, q^{+B)}\right], \\
G^{+\hat{-} 3} & =G^{+(A B C)} u_{A}^{\hat{\bar{A}}} u_{\bar{B}}^{\hat{\bar{A}}} u_{C}^{\hat{\bar{C}}}, & G^{+(A B C)} & =-\frac{1}{3}\left[\mathcal{D}^{(A B}, q^{+C)}\right] .
\end{aligned}
$$

When deducing these solutions, we made use of the reduction relations

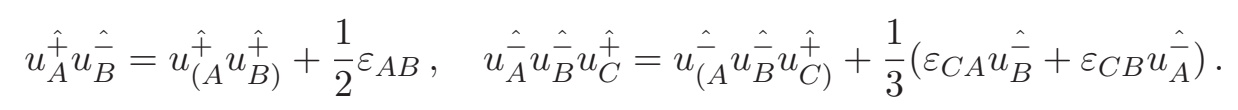

Eq. (7.28a) also implies

$$
\partial^{\hat{+} \hat{+}} W^{+a}=0,
$$

which means independence of $W^{+a}$ of the hatted harmonics. 
Thus, we have fully fixed the $u_{A}^{\hat{+}}, u_{B}^{\hat{\bar{B}}}$ dependence in the $\theta^{\hat{+}}$ expansion (7.14) of $\phi^{+\hat{+}}$. At this stage, it is instructive to write $\phi^{+\hat{+}}$ in the form which takes into account the explicit solutions given above,

$$
\begin{aligned}
& \phi^{+\hat{+}}=q^{+A} u_{A}^{\hat{+}}-\theta_{a}^{\hat{+}} W^{+a}-i \theta_{a}^{\hat{+}} \theta_{b}^{\hat{+}} \hat{\nabla}^{a b} q^{+A} u_{A}^{\hat{\bar{C}}}-\frac{1}{2} \Psi^{\hat{+} 3 d}\left[\varphi_{d}^{A}, q^{+B}\right] u_{A}^{\hat{\bar{A}}} u_{B}^{\hat{\bar{C}}} \\
& -\frac{1}{3} \Psi^{\hat{+} 4}\left[\mathcal{D}^{A B}, q^{+C}\right] u_{A}^{\hat{\bar{C}}} u_{B}^{\hat{-}} u_{C}^{\hat{\bar{C}}} \text {. }
\end{aligned}
$$

Now we are ready to explore the conditions imposed by the second harmonic constraint (7.28b). It implies

$$
\nabla^{++} q^{+\hat{+}}=0, \nabla^{++} W^{+a}=0, \nabla^{++} \hat{\nabla}^{a b} q^{+\hat{-}}=0, \nabla^{++} G_{d}^{+(A B)}=\nabla^{++} G^{+(A B C)}=0 .
$$

The first of these equations is recognized as the equation of motion for the hypermultiplet, so already at this step we can identify $q^{+A}$ with the $\mathcal{N}=(1,0)$ hypermultiplet superfield of the previous sections. Its analyticity follows from the G-analyticity condition $(7.9 \mathrm{~b})$ (see below). The second constraint coincides with (3.38). The third harmonic equation in (7.38) is satisfied as a consequence of the first one and (7.27). The last two equations are satisfied as a consequence of the first equation and the constraints (7.23) and (7.25).

Now we can come back to the problem of the ultimate fixing of the spinor connection $\mathcal{A}_{a}^{+}$. This fixing is accomplished by the constraint (7.2a). Like in the case of $\tilde{V}^{++}$and the constraint (7.3), eq. (7.2a) eliminates all the negatively charged components in the expansion (7.11) [with the condition (7.13)], except for the first term $f_{a}^{+\hat{-} b}$,

$$
f_{a}^{+\hat{\mathcal{\prime}} b c}=f_{d}^{+\hat{-} 3}=f_{a}^{+\hat{-} 4}=0,
$$

whereas $f_{a}^{+\hat{-} b}$ is fixed as

$$
f_{a}^{+\hat{-} b}=-\delta_{a}^{b} q^{+A} u_{A}^{\hat{-}} .
$$

Simultaneously we obtain a few differential conditions relating the $\mathcal{N}=(1,0)$ components of $\phi^{+\hat{+}}$ to those of $V^{\hat{+} \hat{+}}$ defined in (7.8). These are as follows:

$$
\begin{aligned}
D_{a}^{+} \hat{\mathcal{A}}^{b c} & =\frac{i}{2}\left(\delta_{a}^{b} W^{+c}-\delta_{a}^{c} W^{+b}\right), \\
D_{a}^{+} \varphi_{d}^{\hat{-}} & =\frac{i}{3} \varepsilon_{a d b c} \hat{\nabla}^{b c} q^{+\hat{-}} . \\
D_{a}^{+} \mathcal{D}^{A B} & -\frac{3}{8}\left[\varphi_{a}^{(A}, q^{+B)}\right]=0 .
\end{aligned}
$$

Like in the previous cases, these extra equations are self-consistency conditions which are identically satisfied for the general solution of all constraints. As we will see, eq. (7.41) plays the especially important role, giving rise to the expression of $W^{+a}$ in terms of the $\mathcal{N}=(1,0)$ analytic potential $V^{++}$.

The final form for the spinor connection $\mathcal{A}_{a}^{+}$that takes into account the solutions (7.39), (7.40) is

$$
\mathcal{A}_{a}^{+}=-\theta_{a}^{\hat{+}} q^{+A} u_{\bar{A}}^{\hat{-}}+\theta_{a}^{\hat{-}} \phi^{+\hat{+}} .
$$


It remains to work out the conditions following from the G-analyticity constraint (7.9b). Using the explicit expressions (7.44), (7.37), we find that (7.9b) amounts to the following set of equations:

$$
\begin{aligned}
D_{a}^{+} q^{+\hat{+}}=0, \quad D_{a}^{+} W^{+b}=\delta_{a}^{b}\left[q^{+\hat{-}}, q^{+\hat{+}}\right] & =-\frac{1}{2} \delta_{a}^{b}\left[q^{+A}, q_{A}^{+}\right], \\
D_{a}^{+} \hat{\nabla}^{c d} q^{+\hat{-}}-\frac{i}{2}\left(\delta_{a}^{c}\left[q^{+\hat{-}}, W^{+d}\right]-\delta_{a}^{d}\left[q^{+\hat{-}}, W^{+c}\right]\right) & =0, \\
{\left[D_{a}^{+} \varphi_{d}^{-(A}, q^{+B)}\right]+\frac{i}{3} \varepsilon_{a d c f}\left[q^{+(A}, \hat{\nabla}^{c f} q^{+B)}\right] } & =0, \\
{\left[D_{a}^{+} \mathcal{D}^{(A B}, q^{+C)}\right]+\frac{3}{8}\left[q^{+(A},\left[\varphi_{a}^{B}, q^{+C)}\right]\right] } & =0 .
\end{aligned}
$$

The first equation in (7.45) provides the standard analyticity condition for the hypermultiplet $q^{+A}$, while the second equation is going to become the equation of motion for the $\mathcal{N}=(1,0)$ analytic potential $V^{++}$. The remaining equations prove to be satisfied as a consequence of the basic equations of motion.

At last, it is straightforward to check that the constraint (7.1a) does not result in any new restrictions and is identically satisfied as a consequence of G-analyticity of $q^{+{ }^{+}}$and the condition (7.9b).

Let us discuss the peculiarities of the realization of the hidden supersymmetry in the considered frame. As usual, to preserve the Wess-Zumino gauge (7.8), one needs to make a compensating gauge transformation. The appropriate gauge parameter is easily found to be

$$
\Lambda_{(1)}^{(\mathrm{comp})}=2 i \epsilon_{a}^{\hat{-}} \theta_{b}^{\hat{+}} \mathcal{A}^{a b}+\frac{3}{2} \varepsilon^{a b c d} \hat{\epsilon_{a}^{-}} \theta_{b}^{\hat{+}} \theta_{c}^{\hat{+}} \varphi_{d}^{\hat{-}}+\frac{4}{3} \varepsilon^{a b c d} \epsilon_{a}^{\hat{-}} \theta_{b}^{\hat{+}} \theta_{c}^{\hat{+}} \theta_{d}^{\hat{+}} \mathcal{D}^{\hat{-}} 2 .
$$

Besides this, one needs to preserve the "short" form of the spinor connection (7.44). The appropriate compensating gauge parameter is

$$
\Lambda_{(2)}^{(\mathrm{comp})}=\epsilon^{-A} q_{A}^{+},
$$

such that the total compensating gauge parameter is

$$
\Lambda^{(\mathrm{comp})}=\Lambda_{(1)}^{(\mathrm{comp})}+\Lambda_{(2)}^{(\mathrm{comp})} .
$$

Correspondingly, the hidden supersymmetry transformations of $V^{\hat{+} \hat{+}}$ and $\mathcal{A}_{a}^{+}$are

$$
\begin{aligned}
\delta V^{\hat{+} \hat{+}} & =-\epsilon_{a}^{\hat{+}} \frac{\partial}{\partial \theta_{a}^{\hat{+}}} V^{\hat{+} \hat{+}}-2 i \epsilon_{a}^{\hat{-}} \theta_{b}^{\hat{+}} \partial^{a b} V^{\hat{+} \hat{+}}+\nabla^{\hat{+} \hat{+}} \Lambda^{(\mathrm{comp})}, \\
\delta \mathcal{A}_{a}^{+} & =-\epsilon_{b}^{\hat{+}} \frac{\partial}{\partial \theta_{b}^{\hat{+}}} \mathcal{A}_{a}^{+}-\hat{\epsilon} \frac{\partial}{\partial \theta_{b}^{\hat{-}}} \mathcal{A}_{a}^{+}-2 i \epsilon_{c}^{\hat{-}} \theta_{b}^{\hat{+}} \partial^{c b} \mathcal{A}_{a}^{+}+\nabla_{a}^{+} \Lambda^{(\mathrm{comp})} .
\end{aligned}
$$

Note that $\Lambda_{(2)}^{(\mathrm{comp})}$ does not contribute to (7.52).

Since all superfields should undergo the same compensating gauge transformation under the hidden supersymmetry, one can wonder what happens in the case of $V^{++}$. Its transformation law looks as

$$
\delta V^{++}=-2 i \epsilon_{a}^{-} \theta_{b}^{+} \partial^{a b} V^{++}+\nabla^{++} \Lambda^{(\mathrm{comp})}
$$

and seemingly contradicts the fact that $V^{++}$should not depend on the hatted coordinates. However, let us look at $\nabla^{++} \Lambda^{\text {(comp) }}$. Using the constraints (7.25) and (7.23), we find

$$
\nabla^{++} \Lambda^{(\mathrm{comp})}=2 i \epsilon_{a}^{\hat{-}} \theta_{b}^{\hat{+}} \partial^{a b} V^{++}+\nabla^{++}\left(\epsilon^{-A} q_{A}^{+}\right) .
$$


The first term cancels the unwanted term in (7.54), while the second term, with taking into account the on-shell condition $\nabla^{++} q^{+A}=0$, yields the already known transformation law of $V^{++}$under the hidden supersymmetry,

$$
\delta V^{++}=\epsilon^{+A} q_{A}^{+} .
$$

In a similar way, by considering the transformation of the superfield $\phi^{+\hat{+}}$ under the hatted supersymmetry, one can derive the hidden supersymmetry transformations of its $\mathcal{N}=(1,0)$ superfield components $q^{+A}$ and $W^{+a}$.

At this stage, we succeeded to express all the involved geometric quantities of the $\mathcal{N}=$ $(1,1)$ gauge theory in terms of the $\mathcal{N}=(1,0)$ superfields appearing in the $\theta^{\hat{+}}$ expansion of $V^{\hat{+} \hat{+}}$ in the WZ gauge (7.8): the hypermultiplet $q^{+A}$ and the $\mathcal{N}=(1,0)$ superfield $W^{+a}$, which is going to become the covariant $\mathcal{N}=(1,0)$ superfield strength considered in the previous sections. It remains to relate the superfields in (7.8) to the known $\mathcal{N}=(1,0)$ superfields in a pure algebraic way, without solving various differential conditions deduced above. This can be achieved by requiring for the vector superfield connections derived in the hatted and unhatted sectors to coincide (our superspace involves hatted and unhatted odd coordinates, but only one set of bosonic coordinates $x^{M}$ ).

\subsection{Identifying vector connections}

Let us now proceed to the vector connections.

We consider first the unhatted sector. Since $\nabla_{a}^{+}$includes $\theta_{a}^{\hat{ \pm}}$, its counterpart $\nabla_{a}^{-}$should also include now such a dependence, ${ }^{17}$ and the same concerns the full $\mathcal{N}=(1,1)$ superfield vector connection. We define $\nabla_{a}^{-}$in the standard way:

$$
\nabla_{a}^{-}:=D_{a}^{-}+\mathcal{A}_{a}^{-}=\left[\nabla^{--}, \nabla_{a}^{+}\right], \quad \mathcal{A}_{a}^{-}=\mathcal{A}_{a}^{-(0)}-\theta_{a}^{\hat{+}} q^{-\hat{-}}+\theta_{a}^{\hat{-}} \nabla^{--} \phi^{+\hat{+}},
$$

where

$$
\nabla^{--}=D^{--}+V^{--},
$$

$V^{--}$is the same as in the previous sections [it is constructed from $V^{++}$by the harmonic zero curvature equation (3.25)] and $\mathcal{A}_{a}^{-(0)}=-D_{a}^{+} V^{--}$. The relevant full superfield vector connection is defined in the standard way:

$$
\begin{aligned}
\left\{\nabla_{a}^{+}, \nabla_{b}^{-}\right\}= & 2 i\left(\partial_{a b}+\mathcal{V}_{a b}\right), \quad \mathcal{V}_{a b}=\frac{1}{2 i}\left(\nabla_{a}^{+} \mathcal{A}_{b}^{-}+D_{b}^{-} \mathcal{A}_{b}^{+}\right) \\
\mathcal{V}_{a b}= & \mathcal{A}_{a b}+\frac{1}{2 i}\left(\theta_{b}^{\hat{+}} D_{a}^{+} q^{-\hat{-}}+\theta_{a}^{\hat{+}} \nabla_{b}^{-} q^{+\hat{-}}-\theta_{b}^{\hat{-}} D_{a}^{+} \nabla^{--} \phi^{+\hat{+}}-\theta_{a}^{\hat{-}} \nabla_{b}^{-} \phi^{+\hat{+}}\right. \\
& +\theta_{a}^{\hat{+}} \theta_{b}^{\hat{+}}\left[q^{+\hat{-}}, q^{-\hat{-}}\right]-\theta_{a}^{\hat{+}} \theta_{b}^{\hat{-}}\left[q^{+\hat{-}}, \nabla^{--} \phi^{+\hat{+}}\right]+\theta_{b}^{\hat{+}} \theta_{a}^{\hat{-}}\left[\phi^{+\hat{+}}, q^{-\hat{-}}\right] \\
& \left.+\theta_{a}^{\hat{-}} \theta_{b}^{\hat{-}}\left[\phi^{+\hat{+}}, \nabla^{--} \phi^{+\hat{+}}\right]\right), \quad \mathcal{A}_{a b}=\frac{1}{2 i} D_{a}^{+} \mathcal{A}_{b}^{-(0)} .
\end{aligned}
$$

It has the restricted $\theta_{a}^{\hat{-}}$ dependence (only the terms of the first and second order in $\theta_{a}^{\hat{-}}$ ), but includes all $\theta_{a}^{\hat{+}}$ monomials.

\footnotetext{
${ }^{17}$ It is thus not the same as $\nabla_{a}^{-}$in $(3.32)$. We have chosen, however, not to invent other notation and hope that this will not lead to confusion.
} 
On the other hand, one can perform an analogous construction for the derivatives with hatted indices. We define the relevant second harmonic connection $V^{\hat{-}}$ - from the hatted flatness relation

$$
D^{\hat{+} \hat{+}} V^{\hat{\mathcal{A}}}-D^{\hat{\mathcal{A}}} V^{\hat{+} \hat{+}}+\left[V^{\hat{+} \hat{+}}, V^{\hat{\mathcal{A}} \hat{-}}\right]=0
$$

and then introduce the hatted spinor and vector connections as

$$
\begin{aligned}
& {\left[\nabla^{\hat{-}} \hat{-}, D^{\hat{+} a}\right]:=\nabla^{\hat{-a}}=D^{\hat{\sim} a}+\mathcal{A}^{\hat{-} a}, \quad \mathcal{A}^{\hat{-} a}=-\frac{\partial}{\partial \theta_{a}^{\hat{a}}} V^{\hat{-}} \hat{\partial},} \\
& \left\{D^{\hat{+} a}, \nabla^{\hat{-} b}\right\}=2 i\left(\partial^{a b}+\hat{\mathcal{V}}^{a b}\right), \quad \hat{\mathcal{V}}^{a b}=\frac{i}{2} \frac{\partial}{\partial \theta_{a}^{\hat{\alpha}}} \frac{\partial}{\partial \theta_{b}^{\hat{-}}} V^{\hat{\mathcal{-}}},
\end{aligned}
$$

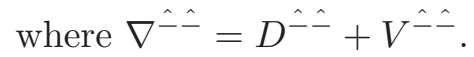

The calculation of $V^{\hat{-} \hat{-}}$ is the most boring part of the whole story. We parametrize the $\theta_{a}^{\hat{-}}$ expansion of $V^{\hat{-} \hat{-}}$ as

$$
V^{\hat{-} \hat{-}}=i \theta_{a}^{\hat{-}} \theta_{b}^{\hat{-}} v^{a b}+\Psi^{\hat{-} 3 d} v_{d}^{\hat{+}}+\Psi^{\hat{-4}} v^{\hat{+} 2} .
$$

All coefficients here are hat-analytic $\mathcal{N}=(1,0)$ superfields, the $u_{A}^{\hat{ \pm}}$ and $\theta_{a}^{\hat{+}}$ dependence of which will be strictly fixed by the corresponding hat-harmonic equations following from (7.60). The possible terms of the zeroth and first orders in $\theta_{a}^{-}$can be shown to vanish by the same mechanism as in the previous examples: their $\theta^{\hat{+}}$ expansions contain only components with negative "hat" charges and these components are killed by the equations like $\partial^{\hat{+} \hat{+}} \omega^{\hat{-} n}=0 \rightarrow \omega^{\hat{-} n}=0$, following from (7.60).

The $\theta_{a}^{-}$expansion of the l.h.s. of the constraint (7.60) contains the Grassmann monomials of the first, second, third and fourth degrees. Equating the corresponding coefficients to zero, we obtain the following set of equations:

$$
\begin{aligned}
& 2 i \theta_{b}^{\hat{+}}\left(\hat{\mathcal{A}}^{b a}-v^{b a}\right)-3 \varepsilon^{a b c d} \theta_{b}^{\hat{+}} \theta_{c}^{\hat{+}} \varphi_{d}^{\hat{-}}-4 \varepsilon^{a b c d} \theta_{b}^{\hat{+}} \theta_{c}^{\hat{+}} \theta_{d}^{\hat{+}} \mathcal{D}^{\hat{-}}=0, \\
& \nabla^{\hat{+}} \hat{+} v^{a b}-3 i \varepsilon^{a b c d} \theta_{c}^{\hat{+}} v_{d}^{\hat{+}}-i \theta_{c}^{\hat{+}} \theta_{d}^{\hat{+}} \partial^{a b} \hat{\mathcal{A}}^{c d}-\Psi^{\hat{+} 3 d} \partial^{a b} \varphi_{d}^{\hat{\bar{c}}}-\Psi^{\hat{+} 4} \partial^{a b} \mathcal{D}^{\hat{-}} 2=0, \\
& \nabla^{\hat{+}} \hat{+} v_{d}^{\hat{+}}+4 \theta_{d}^{\hat{+}} v^{\hat{+} 2}=0, \\
& \nabla^{\hat{+}} v^{\hat{+} 2}=0 .
\end{aligned}
$$

To solve eqs. (7.64)-(7.67), one expands the corresponding unknowns over $\theta_{a}^{\hat{+}}$ and then fix the $u_{A}^{\hat{f}}$ dependence of the coefficients by these equations. For instance, we write

$$
v^{\hat{+} 2}=v_{(0)}^{\hat{+} 2}+\theta_{a}^{\hat{+}} v^{\hat{+} a}+\theta_{a}^{\hat{+}} \theta_{b}^{\hat{+}} w^{a b}+\Psi^{\hat{+} 3 d} v_{d}^{\hat{-}}+\Psi^{\hat{+} 4} v^{\hat{-} 2},
$$

and obtain from (7.67) the following equations and their solutions

$$
\begin{gathered}
\partial^{\hat{+} \hat{+}} v_{(0)}^{\hat{+} 2}=0 \quad \Rightarrow \quad v_{(0)}^{\hat{+} 2}=v^{(A B)} u_{A}^{\hat{+}} u_{B}^{\hat{+}}, \\
\partial^{\hat{+} \hat{+}} v^{\hat{+} a}=0 \quad \Rightarrow \quad v^{\hat{+} a}=v^{a A} u_{A}^{\hat{+}}, \\
\partial^{\hat{+} \hat{+}} w^{a b}+i \hat{\nabla}^{a b} v_{(0)}^{\hat{+} 2}=0 \quad \Rightarrow \quad w^{a b}=w_{0}^{a b}-i \hat{\nabla}^{a b} v^{(A B)} u_{A}^{\hat{+}} u_{B}^{\hat{-}}, \\
\partial^{\hat{+} \hat{+}} v_{d}^{\hat{-}}-\frac{i}{6} \varepsilon_{d a b c} \hat{\nabla}^{a b} v^{\hat{+} c}+\left[\varphi_{d}^{\hat{-}}, v_{(0)}^{\hat{+} 2}\right]=0, \\
\partial^{\hat{+} \hat{+}} v^{\hat{-} 2}+\frac{i}{24} \varepsilon_{a b c d} \hat{\nabla}^{a b} w^{c d}+\left[\mathcal{D}^{\hat{-} 2}, v_{(0)}^{\hat{+} 2}\right]-\frac{1}{4}\left\{\varphi_{a}^{\hat{-}}, v^{\hat{+} a}\right\}=0 .
\end{gathered}
$$


Eq. (7.72) has the following solution,

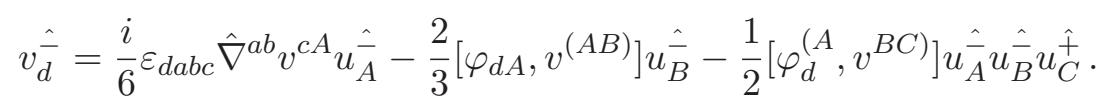

Eq. (7.73) yields both the solution for $v^{\hat{-} 2}$,

$$
\begin{aligned}
v^{\hat{-} 2}= & \frac{1}{2}\left(\left[v^{B(A}, D_{B}^{D)}\right]-\frac{1}{24} \varepsilon_{a b c d} \hat{\nabla}^{a b} \hat{\nabla}^{c d} v^{A D}+\frac{1}{8}\left\{\varphi_{d}^{(A}, v^{d D)}\right\}\right) u_{\bar{A}} u_{D}^{\hat{\bar{D}}} \\
& -\frac{1}{3}\left[D^{(A B}, v^{C D)}\right] \hat{\overline{\bar{A}}} u_{B}^{\hat{\bar{c}}} u_{C}^{\hat{\bar{C}}} u_{D}^{\hat{+}},
\end{aligned}
$$

and the additional self-consistency condition

$$
\left[D^{A B}, v_{A B}\right]+\frac{i}{8} \varepsilon_{a b c d} \hat{\nabla}^{a b} w_{0}^{c d}-\frac{3}{8}\left\{\varphi_{d B}, v^{d B}\right\}=0 .
$$

The remaining equations can be solved analogously. Instead of writing the analogs of the equations (7.69)-(7.73), we will present their solutions, omitting various self-consistency constraints which are identically satisfied on the final full solution.

We start with the equation (7.66). We have

$$
v_{d}^{\hat{+}}=v_{(0) d}^{\hat{+}}+\theta_{b}^{\hat{+}} v_{d}^{b}+\theta_{a}^{\hat{+}} \theta_{b}^{\hat{+}} v_{d}^{\hat{-}[a b]}+\Psi^{\hat{+} 3 d} v_{d b}^{\hat{-} 2}+\Psi^{\hat{+} 4} v_{d}^{\hat{-} 3} .
$$

The solution is

$$
\begin{aligned}
& v_{(0) d}^{\hat{+}}=v_{d}^{A} u_{A}^{\hat{+}}, \quad v_{d}^{b}=v_{0 d}^{b}-4 \delta_{d}^{b} v^{(A B)} u_{A}^{\hat{+}} u_{B}^{\hat{-}}, \\
& v_{d}^{\hat{-}[a b]}=-\left[i \hat{\nabla}^{a b} v_{d}^{A}+2\left(\delta_{d}^{a} v^{b A}-\delta_{d}^{b} v^{a A}\right)\right] u_{A}^{\hat{\bar{A}}} \text {, } \\
& v_{d b}^{\hat{-2}}=v_{d b}^{(A B)} u_{A}^{\hat{\bar{c}}} u_{B}^{\hat{\bar{B}}}, \quad v_{d b}^{(A B)}=-\frac{1}{2}\left\{\varphi_{b}^{(A}, v_{d}^{B)}\right\}+i \frac{2}{3} \varepsilon_{d b c f} \hat{\nabla}^{c f} v^{(A B)} \text {, } \\
& v_{d}^{\hat{-} 3}=v_{d}^{(A B C)} u_{A}^{\hat{\bar{A}}} u_{B}^{\hat{\bar{B}}} u_{C}^{\hat{\bar{C}}}, \quad v_{d}^{(A B C)}=\frac{1}{2}\left[v^{(A B}, \varphi_{d}^{C)}\right]+\frac{1}{3}\left[v_{d}^{(A}, \mathcal{D}^{B C)}\right] .
\end{aligned}
$$

One more important relation following from (7.66) is

$$
\varepsilon_{a b c d} w_{0}^{c d}+\frac{i}{4} \varepsilon_{f b c d} \hat{\nabla}^{c d} v_{0 a}^{f}+\frac{1}{2}\left\{\varphi_{b D}, v_{a}^{D}\right\}=0 .
$$

We now turn to (7.65). Once again, we expand

$$
v^{[a b]}=v_{(0)}^{[a b]}+\theta_{d}^{\hat{+}} v^{\hat{-} d[a b]}+\theta_{c}^{\hat{+}} \theta_{d}^{\hat{+}} v^{\hat{-} 2[c d][a b]}+\Psi^{\hat{+} 3 d} v_{d}^{\hat{-} 3[a b]}+\Psi^{\hat{+} 4} v^{\hat{-} 4[a b]} .
$$

The solution is

$$
\begin{array}{rlrl}
v_{(0)}^{[a b]} & =v_{0}^{[a b]}, & v_{d}^{\hat{-}-3[a b]} & =v^{\hat{-} 4[a b]}=0, \\
v^{\hat{-} d[a b]} & =3 i \varepsilon^{d a b c} v_{c}^{A} \hat{u_{A}^{\hat{A}}}, & v^{\hat{-} 2[c d][a b]}=-6 i \varepsilon^{c d a b} v^{A B} u_{A}^{\hat{\bar{A}}} u_{B}^{\hat{\bar{B}}}, \\
\varepsilon^{a b[c f} v_{0 g}^{d]} & =\frac{1}{3}\left(\hat{\nabla}^{c d} v_{0}^{[a b]}-\partial^{a b} \hat{\mathcal{A}}^{c d}\right) . & &
\end{array}
$$


An important consequence of (7.65) is also the relation

$$
\partial^{a b} \varphi_{d}^{A}+\left[v_{0}^{[a b]}, \varphi_{d}^{A}\right]+\frac{1}{2} \varepsilon^{a b c f} \varepsilon_{c d u g}\left[\hat{\nabla}^{u g} v_{f}^{A}-2 i\left(\delta_{f}^{u} v_{0}^{g A}-\delta_{f}^{g} v_{0}^{u A}\right)\right]=0 .
$$

The most crucial is eq. (7.64). It gives

$$
v_{0}^{[a b]}=\hat{\mathcal{A}}^{[a b]}, \quad v_{b}^{A}=-\frac{1}{2} \varphi_{b}^{A}, \quad v^{(A B)}=\frac{1}{3} \mathcal{D}^{(A B)} .
$$

Now, substituting all this into (7.87), we can determine $v_{0}^{b A}$,

$$
v_{0}^{b A}=\frac{i}{4} \hat{\nabla}^{b a} \varphi_{a}^{A} .
$$

Using (7.86) and (7.82), we can also express $w_{0}^{[a b]}$ and $v_{0 b}^{d}$ through the basic superfields $\hat{\mathcal{A}}^{[a b]}, \varphi_{b}^{A}$ and $\mathcal{D}^{(A B)}$. Thus, we obtain the full solution for $V^{\hat{\mathcal{-}}}$.

Now we are ready to explicitly construct the full superfield vector connection $\hat{\mathcal{V}}^{a b}$. Using the definition (7.62), we obtain

$$
\hat{\mathcal{V}}^{a b}=v^{[a b]}+3 i \epsilon^{a b c d} \theta_{c}^{\hat{-}} v_{d}^{\hat{+}}+6 i \epsilon^{a b c d} \theta_{c}^{\hat{-}} \theta_{d}^{\hat{-}} v^{\hat{+} 2} .
$$

The crucial requirement now is that this connection is related to the connection $\mathcal{V}^{a b}$ in the sector of "unhatted" spinor derivatives as

$$
\mathcal{V}_{a b}=\frac{1}{2} \epsilon_{a b c d} \hat{\mathcal{V}}^{c d}
$$

Comparing two expressions in the zeroth order in $\theta_{c}^{\hat{-}}$, we immediately find

$$
\hat{\mathcal{A}}^{a b}=\frac{1}{2} \epsilon^{a b c d} \mathcal{A}_{c d}, \quad \varphi_{a}^{\hat{-}}=-\frac{1}{3} D_{a}^{+} q^{-\hat{-}}, \quad \mathcal{D}^{A B}=\frac{1}{8}\left[q^{+(A}, q^{-B)}\right],
$$

which, being substituted into the basic superfields $V^{\hat{+} \hat{+}}$ and $\phi^{+\hat{+}}(7.8),(7.37)$, precisely reproduce the solution (6.27) and (6.28) that we have presented in the previous section. Comparing the coefficients of the next terms of expansion in $\theta_{c}^{-}$in the equation (7.91) gives relations that are identically satisfied, when taking into account the $\mathcal{N}=(1,0)$ equations of motion and the G-analyticity conditions, and so does not produce new constraints.

Note that after the identification (7.92), the relation (7.41) becomes equivalent to (3.34). It gives the expression (3.35) of $W^{+a}$ in terms of $V^{--}$.

\section{On-shell $\mathcal{N}=(1,1)$ supersymmetric actions}

\subsection{Invariant actions: $d=8$}

We can now use the techniques developed in section 6 and 7 to write down the actions invariant under the extended $\mathcal{N}=(1,1)$ on-shell supersymmetry. The original $d=4$ action (4.14) is off-shell invariant. For $d=6$, the invariants non-vanishing on shell are absent. Dimension 8 is the first nontrivial case. 
Consider the density

$$
\mathcal{L}_{(1,1)}^{+4}=-\operatorname{Tr} \int d \hat{\zeta}^{(-4)} \frac{1}{4}\left(\phi^{+\hat{+}}\right)^{4}
$$

where the hatted analytic superspace measure $d \hat{\zeta}^{(-4)}$ is defined as $d \hat{\zeta}^{(-4)}=d \hat{u}\left(\hat{D}^{-}\right)^{4}$ (in contrast to $d \zeta^{(-4)}$, it does not involves $d^{6} x$ ). Bearing in mind the property (7.9b),

$$
\nabla_{a}^{+} \phi^{+\hat{+}}=D_{a}^{+} \phi^{+\hat{+}}+\left[\mathcal{A}_{a}^{+}, \phi^{+\hat{+}}\right]=0
$$

where $\mathcal{A}_{a}^{+}$is given in (7.44), and the fact that the commutator term does not contribute under the trace, we derive that the Lagrangian $(8.1)$ is $\mathcal{N}=(1,0)$ analytic:

$$
D_{a}^{+} \mathcal{L}_{(1,1)}^{+4}=0
$$

This analyticity holds only on shell since the constraint ( $7.9 \mathrm{~b})$ necessarily implies the second equation of motion in (7.45).

It is easy to see that the integral (8.1) is shifted by a total derivative under the $\mathcal{N}=(0,1)$ transformations $(6.30)$. Indeed, the integrand transforms as

$$
\delta \operatorname{Tr}\left[\frac{1}{4}\left(\phi^{+\hat{+}}\right)^{4}\right]=-\epsilon_{a}^{\hat{+}} \frac{\partial}{\partial \theta_{a}^{\hat{+}}} \operatorname{Tr}\left[\frac{1}{4}\left(\phi^{+\hat{+}}\right)^{4}\right]-2 i \epsilon_{a}^{\hat{-}} \theta_{b}^{\hat{+}} \partial^{a b} \operatorname{Tr}\left[\frac{1}{4}\left(\phi^{+\hat{+}}\right)^{4}\right],
$$

and hence

$$
\delta \mathcal{L}_{(1,1)}^{+4}=-2 i \partial^{a b} \operatorname{Tr} \int d \hat{\zeta}^{(-4)}\left(\epsilon_{a}^{\hat{-}} \theta_{b}^{\hat{+}} \frac{1}{4}\left(\phi^{+\hat{+}}\right)^{4}\right)
$$

The action

$$
S_{(1,1)}=\int d \zeta^{(-4)} \mathcal{L}_{(1,1)}^{+4}
$$

is clearly invariant.

To express (8.1) in terms of $\mathcal{N}=(1,0)$ superfields, we need to substitute there the explicit expression (6.28) for $\phi^{+\hat{+}}$ and to integrate over $d \hat{\zeta}^{(-4)} d \hat{u}$, using (3.22) (with the capital SU(2) indices $A, B, \ldots$ instead of $i, j, \ldots)$. Doing this, we reproduce the result (5.34) quoted above.

Though it is not at all seen in the expression (5.34), we expect that the Lagrangian expressed in components is invariant under the permutation $\lambda \leftrightarrow \psi$.

The Lagrangian (5.34) represents a $\mathcal{N}=(1,1)$ generalization of the $\mathcal{N}=(1,0)$ supersymmetric single-trace Lagrangian (5.21) involving the vector supermultiplet. It is trivial to generalize in a similar way the double-trace Lagrangian (5.24). It is sufficient to consider the density

$$
\hat{\mathcal{L}}_{(1,1)}^{+4}=-\frac{1}{4} \int d \hat{\zeta}^{(-4)} \operatorname{Tr}\left(\phi^{+\hat{+}}\right)^{2} \operatorname{Tr}\left(\phi^{+\hat{+}}\right)^{2}
$$


and perform the integrals over the hatted variables. The result is expressed in terms of the $\mathcal{N}=(1,0)$ superfields as follows:

$$
\begin{aligned}
\hat{\mathcal{L}}_{(1,1)}^{+4}= & \frac{1}{4} \varepsilon_{a b c d} \operatorname{Tr}\left(W^{+a} W^{+b}\right) \operatorname{Tr}\left(W^{+c} W^{+d}+2 i q^{+A} \nabla^{c d} q_{A}^{+}\right) \\
& -\frac{1}{2} \operatorname{Tr}\left(q^{+A} \nabla^{a b} q_{A}^{+}\right) \operatorname{Tr}\left(q^{+B} \nabla_{a b} q_{B}^{+}\right)+\frac{1}{12} \partial^{a b} \operatorname{Tr}\left(q^{+A} q^{+B}\right) \partial_{a b} \operatorname{Tr}\left(q_{A}^{+} q_{B}^{+}\right) \\
& +\operatorname{Tr}\left(q^{+A} W^{+a}\right) \operatorname{Tr}\left\{D_{a}^{+} q_{A}^{-}\left(q^{+}\right)^{2}-2 i W^{+b} \nabla_{b a} q_{A}^{+}\right\} \\
& +\frac{1}{3} \operatorname{Tr}\left(q^{+A} q^{+B}\right) \operatorname{Tr}\left\{\left(q^{+}\right)^{2}\left[q_{(A}^{+}, q_{B)}^{-}\right]-\nabla^{a b} q_{A}^{+} \nabla_{a b} q_{B}^{+}-W^{+a}\left[D_{a}^{+} q_{(A}^{-}, q_{B)}^{+}\right]\right\} .
\end{aligned}
$$

Thus, the nontrivial on-shell $d=8$ invariants exist. Still the perturbative expansion for the amplitudes in the theory (4.14) does not involve divergences at the two-loop level. The matter is that these invariants do not possess the full off-shell $\mathcal{N}=(1,0)$ supersymmetry, which the physically relevant counterterms should obey. Indeed, we have in our disposal the off-shell $\mathcal{N}=(1,0)$ harmonic superfield description, which implies the existence of the gauge-covariant $\mathcal{N}=(1,0)$ supergraph techniques, such that all the relevant counterterms enjoy this off-shell symmetry.

\subsubsection{Gauge non-invariant off-shell supersymmetric realization}

Our remark is that one still can write an off-shell supersymmetric $d=8$ action, if renouncing the requirement of gauge invariance. The corresponding density reads

$$
\begin{aligned}
\hat{\mathcal{L}}^{+4}= & \operatorname{Tr}_{(S)}\left\{\frac{1}{4} \varepsilon_{a b c d} W^{+a} W^{+b} W^{+c} W^{+d}+3 i q^{+A} \nabla_{a b} q_{A}^{+} W^{+a} W^{+b}-q^{+A} \nabla_{a b} q_{A}^{+} q^{+B} \nabla^{a b} q_{B}^{+}\right. \\
& -W^{+a}\left[D_{a}^{+} q_{A}^{-}, q_{B}^{+}\right] q^{+A} q^{+B}-\frac{1}{2}\left[q^{+C}, q_{C}^{+}\right]\left[q_{A}^{-}, q_{B}^{+}\right] q^{+A} q^{+B} \\
& +\left(F^{++}+\frac{1}{2}\left[q^{+A}, q_{A}^{+}\right]\right)\left(2 i \mathcal{A}_{a b} W^{+a} W^{+b}-2 F^{++} \mathcal{A}_{a b} \mathcal{A}^{a b}\right. \\
& \left.\left.-3 q^{+B} \nabla_{a}^{-} q_{B}^{+} W^{+a}+3 q^{+B} \nabla^{--} q_{B}^{+} F^{++}+\left[q_{B}^{-}, q_{C}^{+}\right] q^{+B} q^{+C}\right)\right\}
\end{aligned}
$$

Indeed, it is not difficult to check that the expression (8.9) is G-analytic off mass shell. One further notices that the density (8.9) coincides with (5.34) modulo the terms proportional to the equations of motion. In other words, (8.9) represents the same counterterm as (5.34); one expression is obtained from another by a field redefinition.

Consider now a deformation of the action (4.14) involving not the density (5.34), but the gauge non-invariant off-shell supersymmetric density (8.9),

$$
S=S^{V q^{+}}+f^{2} \int d \zeta^{(-4)} \hat{\mathcal{L}}^{+4}+f^{6} S_{2}+\ldots
$$

To order $f^{2}$, the standard gauge transformation of the complete action reads

$\delta S=\frac{1}{f^{2}} \operatorname{Tr}_{(s)} \int d \zeta^{(-4)}\left[\left(F^{++}+\frac{1}{2}\left[q^{+A}, q_{A}^{+}\right]\right)\left(\delta V^{++}+2 i f^{4} \partial_{a b} \Lambda\left(W^{+a} W^{+b}-2 F^{++} \mathcal{A}^{a b}\right)\right)\right]$, 
which vanishes on shell, but not off shell. On the other hand, one can notice that the action (8.10) is invariant under the modified gauge transformation

$$
\delta V^{++}=\nabla^{++} \Lambda-2 i f^{4} T\left(\partial_{a b} \Lambda\left(W^{+a} W^{+b}-2 F^{++} A^{a b}\right)\right)+\mathcal{O}\left(f^{8}\right),
$$

where $T(\cdots)$ stands for the symmetrized product projected on the Lie algebra,

$$
T\left(X_{1} X_{2} X_{3}\right)=\frac{1}{6} T^{p} \operatorname{Tr}\left[T^{p}\left(X_{1}\left\{X_{2}, X_{3}\right\}+X_{2}\left\{X_{3}, X_{1}\right\}+X_{3}\left\{X_{1}, X_{2}\right\}\right)\right],
$$

with the generators $T^{p}$ normalized by $\operatorname{Tr}\left(T^{p} T^{q}\right)=\delta^{p q}$.

This gauge transformation preserves the G-analyticity of $V^{++}$. The algebra of the modified gauge transformations closes,

$$
\left(\delta\left(\Lambda_{1}\right) \delta\left(\Lambda_{2}\right)-\delta\left(\Lambda_{2}\right) \delta\left(\Lambda_{2}\right)\right) V^{++}=\delta\left(\left[\Lambda_{1}, \Lambda_{2}\right]\right) V^{++}+\mathcal{O}\left(f^{8}\right) .
$$

The situation when the action representing an infinite series (8.10) is invariant under the modified gauge transformations (8.12), which also are given by an infinite series, is exactly the same as what happens for off-shell supersymmetry, when choosing the gaugeinvariant realization, cf. eqs. (2.12), (2.13) and their discussion in section 2.

Alternatively, one can restore the standard realization of gauge transformations by redefining the superfield $V^{++}$in such a way that

$$
\left.V^{++} \rightarrow V^{++}+2 i f^{4} T\left(\mathcal{A}_{a b} W^{+a} W^{+b}-F^{++} \mathcal{A}_{a b} \mathcal{A}^{a b}\right)\right)+\mathcal{O}\left(f^{8}\right) .
$$

The modified $V^{++}$is not analytic anymore,

$$
D_{a}^{+} V^{++}=\left[D_{a}^{+}, \nabla^{++}\right]=-f^{4} \varepsilon_{a b c d} T\left(W^{+b} W^{+c} W^{+d}\right)+\mathcal{O}\left(f^{8}\right) .
$$

The nonzero commutator (8.16) is related to the non-zero curvature constraints derived in [43] in the ordinary $\mathcal{N}=(1,0)$ superspace formalism.

\subsection{Invariant actions: $d=10$}

We again start with the invariants in the pure gauge sector.

One can write two different off-shell $\mathcal{N}=(1,0)$ supersymmetric and gauge invariant Lagrangians of canonical dimension 10. One of them is known as the single-trace invariant; the corresponding action reads

$$
S_{1}^{(10)}=\int d Z \varepsilon_{a b c d} \operatorname{Tr}\left(W^{+a} W^{-b} W^{+c} W^{-d}\right) .
$$

Any $d=10$ invariant with a different ordering of the covariant superfield strengths is reduced to (8.17) by integrating by parts with respect to the harmonic derivatives [using the relations (3.38)]. One can derive in this way the following convenient representation for (8.17),

$$
S_{1}^{(10)}=\frac{1}{3} \int d Z \varepsilon_{a b c d} \operatorname{Tr}\left(\left\{W^{+a}, W^{-b}\right\}\left\{W^{+c}, W^{-d}\right\}\right) .
$$


This form of the $d=10$ invariant implies, in particular, that all possible $q^{+A}$-dependent terms completing this off-shell $\mathcal{N}=(1,0)$ invariant to an on-shell $\mathcal{N}=(1,1)$ invariant should represent a trace of the product either of two anticommutators or of two commutators. Thus, they should vanish in the abelian limit together with the term (8.18).

There is also the double trace invariant,

$$
S_{2}^{(10)}=\int d Z \epsilon_{a b c d} \operatorname{Tr}\left(W^{+a} W^{-b}\right) \operatorname{Tr}\left(W^{+c} W^{-d}\right) .
$$

Its uniqueness can be as well proved via integrating by parts and taking advantage of the relations (3.38). In the abelian limit, (8.19) vanishes by the same token as (8.17).

The difference of the invariants (8.17) and (8.19) from (5.21) and (5.24) is that the harmonic charge of the integrand in the former is zero, and the integral now goes over the whole superspace rather than its analytic subspace. This brings about two additional powers of mass in the component Lagrangians. Another crucial difference is that (8.17) and (8.19) are $\mathcal{N}=(1,0)$ supersymmetric off shell, whereas (5.21) and (5.24) are supersymmetric only on shell.

To construct the possible on-shell $\mathcal{N}=(1,1)$ completion of (8.17) and (8.19) one can proceed in the spirit of section 5 . Namely, one can add to these expressions all possible $\mathcal{N}=(1,0)$ superfield invariants of dimension $d=10$ with hypermultiplets and require the sum to be invariant up to a total derivative under the $\mathcal{N}=(0,1)$ transformations (4.28)(4.31) on the mass shell (4.17), (4.8). In section 5 , we managed to carry out this program for the single-trace $d=8$ invariant, but, for $d=10$, this turns out to be an extremely difficult task. The calculations are much more simple, if using the on-shell $\mathcal{N}=(1,1)$ harmonic superspace formalism.

We introduce the superfield

$$
\begin{aligned}
\phi^{-\hat{+}}=\nabla^{--} \phi^{+\hat{+}}= & q^{-\hat{+}}-\theta_{a}^{\hat{+}} W^{-a}-i \theta_{a}^{\hat{+}} \theta_{b}^{\hat{+}} \nabla^{a b} q^{-\hat{-}}+\frac{1}{6} \varepsilon^{a b c d} \theta_{a}^{\hat{+}} \theta_{b}^{\hat{+}} \theta_{c}^{\hat{+}}\left[D_{d}^{+} q^{-\hat{-}}, q^{-\hat{-}}\right] \\
& -\frac{1}{24} \varepsilon^{a b c d} \theta_{a}^{\hat{+}} \theta_{b}^{\hat{+}} \theta_{c}^{\hat{+}} \theta_{d}^{\hat{+}}\left[q^{-\hat{-}},\left[q^{-\hat{-}}, q^{+\hat{-}}\right]\right]
\end{aligned}
$$

It satisfies the constraints,

$$
D_{a}^{\hat{+}} \phi^{-\hat{+}}=\nabla_{a}^{-} \phi^{-\hat{+}}=\nabla^{--} \phi^{-\hat{+}}=\nabla^{\hat{+} \hat{+}} \phi^{-\hat{+}}=0,
$$

where

$$
\nabla_{a}^{-}=D_{a}^{-}-D_{a}^{+} V^{--}-\theta_{a}^{\hat{+}} q^{-\hat{-}}+\theta^{-} \phi^{-\hat{+}}
$$

[cf. (7.56)]. The superfield (8.20) appears in the anticommutator

$$
\left\{D^{\hat{+} b}, \nabla_{a}^{-}\right\}=\delta_{a}^{b} \phi^{-\hat{+}},
$$

which can be obtained by applying $\nabla^{--}$to both sides of the constraint (7.1c).

In the full analogy with $(6.30)$, the $\mathcal{N}=(0,1)$ variation of $(8.20)$ is a combination of a total space-time derivative, total $\theta$ derivative and the commutator term. The latter involves the same compensating superfield $\Lambda^{\text {comp }}$ as in (6.30). 
With the superfields $\phi^{+\hat{+}}$ and $\phi^{-\hat{+}}$ in hand, it is rather clear how to define the two $d=10 \mathcal{N}=(1,1)$ invariant actions generalizing (8.17) and (8.19). They are:

$$
S_{1}^{(10)}=\operatorname{Tr} \int d Z d \hat{\zeta}^{(-4)}\left(\phi^{+\hat{+}}\right)^{2}\left(\phi^{-\hat{+}}\right)^{2}
$$

and

$$
S_{2}^{(10)}=-\int d Z d \hat{\zeta}^{(-4)} \operatorname{Tr}\left(\phi^{+\hat{+}} \phi^{-\hat{+}}\right) \operatorname{Tr}\left(\phi^{+\hat{+}} \phi^{-\hat{+}}\right),
$$

where the minus sign in (8.25) was chosen for further convenience.

In contrast to (8.1), these invariants vanish in the abelian limit (in agreement with the fact that (8.17) and (8.19) vanish in this limit). This property can be made manifest for (8.24) by rewriting it as

$$
S_{1}^{(10)}=-\frac{1}{6} \operatorname{Tr} \int d Z d \hat{\zeta}^{(-4)}\left[\phi^{+\hat{+}}, \phi^{-\hat{+}}\right]\left[\phi^{+\hat{+}}, \phi^{-\hat{+}}\right] .
$$

The single trace invariant can also be written as a full superspace integral

$$
S_{1}^{(10)} \sim \operatorname{Tr} \int d Z d \hat{Z} \phi^{+\hat{+}} \phi^{-\hat{-}}, \quad \phi^{-\hat{-}}=\nabla^{\hat{-}} \phi^{-\hat{+}} .
$$

To show this, we represent

$$
d \hat{Z}=d \hat{\zeta}^{(-4)}\left(D^{\hat{+}}\right)^{4}, \quad\left(D^{\hat{+}}\right)^{4}:=-\frac{1}{24} \varepsilon_{a b c d} D^{a \hat{+}} D^{b \hat{+}} D^{c \hat{+}} D^{d \hat{+}}
$$

and use the hat-analyticity of $\phi^{+\hat{+}}$ and $\phi^{-\hat{+}}$, as well as the relations

$$
D^{d \hat{+}} \phi^{-\hat{-}}=-\nabla^{d \hat{-}} \phi^{-\hat{+}}, \quad D^{c \hat{+}} D^{d \hat{+}} \phi^{-\hat{-}}=-2 i \nabla^{c d} \phi^{-\hat{+}}
$$

to bring (8.27) in the form

$$
\sim \frac{i}{12} \int d \hat{\zeta}^{(-4)} \operatorname{Tr}\left(\phi^{+\hat{+}} \varepsilon_{a b c d} D^{a \hat{+}} D^{b \hat{+}} \nabla^{c d} \phi^{-\hat{+}}\right) .
$$

After that we rewrite $\nabla^{c d}$ through the unhatted covariant derivatives as

$$
\nabla^{c d}=\frac{1}{2} \varepsilon^{c d a b} \nabla_{a b}, \quad \nabla_{a b}=\frac{1}{2 i}\left\{\nabla_{a}^{+}, \nabla_{b}^{-}\right\}
$$

and then pull out the remaining two hatted derivatives $D^{a \hat{+}} D^{b \hat{+}}$ to the right through the anticommutator (8.31), using the constraint (7.1c) and its corollary (8.23). The result is

$$
\int d \hat{Z} \operatorname{Tr}\left(\phi^{+\hat{+}} \phi^{-\hat{-}}\right)=\int d \hat{\zeta}^{(-4)} \operatorname{Tr}\left(\left[\phi^{+\hat{+}}, \phi^{-\hat{+}}\right]\left[\phi^{+\hat{+}}, \phi^{-\hat{+}}\right]\right) .
$$

On the other hand, the double trace invariant cannot be written as a full-superspace integral and can be considered as a 1/4 BPS protected operator. This allows one to explain the absence of the associated logarithmic divergence in the pure spinor formalism [22-24]. However, it is not yet sufficient to prove the non-renormalization theorem in the standard quantum field theory framework. Eq. (8.25) is a full-superspace integral over $\mathcal{N}=(1,0)$ 
harmonic superspace, and is á priori allowed by the harmonic superspace Feynman rules. One may anticipate nonetheless that the Ward identities for the non-linearly realized extra supersymmetry would permit to rule it out as an allowed counterterm. The integrand in (8.25) is invariant with respect to the transformations (4.15) modulo a total derivative in $\mathcal{N}=(1,0)$ harmonic superspace and taking into account the equations of motion. The variation of a total derivative with respect to (4.15) gives again a total derivative, and one gets in this way a chain of co-forms associated to a given supersymmetry invariant (see section 5.3 of [38]). One shows then in the framework of algebraic renormalization [20] that the cohomology class associated to this chain of co-forms must be compatible with the cohomology class associated to the classical (dimension 4) Lagrangian. In this way, one would combine the constraints following from the $\mathcal{N}=(1,0)$ harmonic superspace Feynman rules and the constraints following from the full $\mathcal{N}=(1,1)$ on-shell supersymmetry of the action in the framework of algebraic renormalization. One knows that neither of these methods, taken separately, is powerful enough to explain the absence of the non-planar divergence at three loops [21]. But we hope that, being combined in this way, they may allow to prove the required non-renormalization theorem. We will not, however, investigate this issue further in this paper.

Let us come back to the explicit form of the $d=10$ invariants in the $\mathcal{N}=(1,0)$ harmonic superspace. It is rather straightforward to perform the integration over $d \hat{\zeta}^{(-4)}$ in the invariant (8.26) and obtain its $\mathcal{N}=(1,0)$ superfield form.

The result of integration can be written as a sum of the three terms

$$
\tilde{S}_{1}^{(10)}=\int d Z\left(\mathcal{L}_{(1)}^{(10)}+\mathcal{L}_{(2)}^{(10)}+\mathcal{L}_{(3)}^{(10)}\right)
$$

where

$$
\begin{aligned}
\mathcal{L}_{(1)}^{(10)}= & \frac{1}{6} \varepsilon_{a b c d} \operatorname{Tr}\left(\left\{W^{+a}, W^{-b}\right\}\left\{W^{+c}, W^{-d}\right\}-2 i\left\{W^{+a}, W^{-b}\right\}\left[q^{-A}, \nabla^{c d} q_{A}^{+}\right]\right. \\
& -\frac{1}{2} \nabla^{a b}\left(q^{-}\right)^{2} \nabla^{c d}\left(q^{+}\right)^{2}+\frac{1}{6} \nabla^{a b}\left[q^{-(A}, q^{+B)}\right] \nabla^{c d}\left[q_{(A}^{-}, q_{B)}^{+}\right] \\
& \left.-\left[q^{+A}, \nabla^{a b} q_{A}^{-}\right]\left[q^{+B}, \nabla^{c d} q_{B}^{-}\right]\right) \\
\mathcal{L}_{(2)}^{(10)}= & \frac{2}{3} \operatorname{Tr}\left\{\left(\left[q^{+A}, W^{-a}\right]-\left[q^{-A}, W^{+a}\right]\right)\left(i\left[W^{+b}, \nabla_{a b} q_{A}^{-}\right]+\frac{1}{3}\left[q^{+B},\left[q_{(B}^{-}, D_{a}^{+} q_{A}^{-}\right)\right]\right]\right\}, \\
\mathcal{L}_{(3)}^{(10)}= & \frac{1}{18} \operatorname{Tr}\left\{[ q ^ { + ( A } , q ^ { - B ) } ] \left(\left[q^{+C},\left[q_{C}^{-},\left[q_{(A}^{+}, q_{B)}^{-}\right]\right]\right]+2\left[q^{+C},\left[q_{A}^{-},\left[q_{(B}^{+}, q_{C)}^{-}\right]\right]\right]\right.\right. \\
& \left.\left.-4\left\{W^{+a},\left[D_{a}^{+} q_{A}^{-}, q_{B}^{-}\right]\right\}-4\left[\nabla^{a b} q_{A}^{+}, \nabla_{a b} q_{B}^{-}\right]\right)\right\} .
\end{aligned}
$$

While deriving (8.34)-(8.36), we essentially used the integration by parts and various onshell conditions like $\nabla^{--} q^{-A}=0$ etc. Perhaps, these expressions can be further simplified by integrating by parts and using some SU(2) Fierz identities. Anyway, it would be very difficult to guess them entirely within the $\mathcal{N}=(1,0)$ superfield formalism.

A good check of the correctness of (8.34)-(8.36) is the verification of the fact that the variation of the first term in (8.34) under the hidden supersymmetry $\hat{\delta} W^{ \pm a}=-2 i \epsilon_{b}^{A} \nabla^{a b} q_{A}^{ \pm}$ [see (4.29)] is canceled (modulo various terms vanishing on-shell) by the $(W)^{3}$ part of the 
variation of the term $\sim W^{2}$ in (8.33). The latter term is assembled from the pieces coming from (8.34) and (8.35) and, after some algebra, is represented as

$$
2 i \operatorname{Tr}\left(\left[\nabla_{a b} q_{A}^{+}, W^{-a}\right]\left[W^{+b}, q^{-A}\right]\right) .
$$

Its variation under $\hat{\delta} q^{ \pm A}=\epsilon_{a}^{A} W^{ \pm a}$ [see (4.28)] exactly cancels the variation of the first term in (8.34).

The double-trace invariant (8.25) can also be straightforwardly cast into the $\mathcal{N}=(1,0)$ superfield form:

$$
\begin{aligned}
S_{2}^{(10)}= & \int d Z\left(\hat{\mathcal{L}}_{(1)}^{(10)}+\hat{\mathcal{L}}_{(2)}^{(10)}+\hat{\mathcal{L}}_{(3)}^{(10)}\right), \\
\hat{\mathcal{L}}_{(1)}^{(10)}= & \varepsilon_{a b c d}\left\{\operatorname{Tr}\left(W^{+a} W^{-b}\right) \operatorname{Tr}\left(W^{+c} W^{-d}\right)-i \operatorname{Tr}\left(W^{+a} W^{+b}\right) \operatorname{Tr}\left(q^{-A} \nabla^{c d} q_{A}^{-}\right)\right\} \\
& +\operatorname{Tr}\left(q^{+A} \nabla^{a b} q_{A}^{+}\right) \operatorname{Tr}\left(q^{-B} \nabla_{a b} q_{B}^{-}\right)-\frac{1}{6} \partial^{a b} \operatorname{Tr}\left(q^{+A} q^{+B}\right) \partial_{a b} \operatorname{Tr}\left(q_{A}^{+} q_{B}^{+}\right), \\
\hat{\mathcal{L}}_{(2)}^{(10)}= & \frac{4}{3} \operatorname{Tr}\left(q^{+A} W^{+a}\right) \operatorname{Tr}\left\{q^{-B}\left[D_{a}^{+} q_{(A}^{-}, q_{B)}^{-}\right]-3 i \nabla_{a b} q_{A}^{-} W^{-b}\right\}, \\
\hat{\mathcal{L}}_{(3)}^{(10)}= & \frac{2}{3} \operatorname{Tr}\left(q^{+A} q^{-B}\right) \operatorname{Tr}\left\{\left[q^{+C}, q_{C}^{-}\right]\left[q_{(A}^{+}, q_{B)}^{-}\right]-2 \nabla^{a b} q_{(A}^{+} \nabla_{a b} q_{B)}^{-}\right. \\
& \left.+2\left[D_{a}^{+} q_{(A}^{-}, q_{B)}^{-}\right] W^{+a}\right\} .
\end{aligned}
$$

As a good self-consistency check, one can verify that, in the abelian limit, the $\mathcal{N}=(1,0)$ superfield Lagrangian in (8.37) is indeed reduced to a total derivative. This check is not trivial because not all terms in (8.38) contain (anti)commutators under the trace [in contrast to $(8.34)-(8.36)]$.

Finally, we want to point out once more that the actions (8.33) and (8.37) respect the off-shell $\mathcal{N}=(1,0)$ supersymmetry, being written in terms of the off-shell $\mathcal{N}=(1,0)$ superfields. They also respect the on-shell $\mathcal{N}=(0,1)$ invariance because they admit an equivalent representation as integrals over the $\mathcal{N}=(1,1)$ harmonic superspace and its non-trivial subspaces supporting a linear realization of both $\mathcal{N}=(1,0)$ and $\mathcal{N}=(0,1)$ supersymmetries. The second supersymmetry becomes nonlinear, when is realized in terms of the $\mathcal{N}=(1,0)$ superfields. To avoid a possible confusion, we note that the $\mathcal{N}=$ $(1,1)$ form $(8.24)$ and $(8.25)$ of the $d=10$ terms already enjoys the off-shell $\mathcal{N}=(1,0)$ supersymmetry. The equations of motion come into play, only when checking the $\mathcal{N}=(0,1)$ invariance of these expressions.

\section{$9 \quad$ Summary and outlook}

In this paper, we applied the off-shell $\mathcal{N}=(1,0)$ and on-shell $\mathcal{N}=(1,1)$ harmonic superspace approaches for constructing higher-dimensional invariants in the six-dimensional $\mathcal{N}=(1,0) \mathrm{SYM}$ and $\mathcal{N}=(1,1) \mathrm{SYM}$ theories. The $\mathcal{N}=(1,1)$ SYM theory constraints were solved in terms of $\mathcal{N}=(1,0)$ harmonic superfields. This allowed us to explicitly construct the full set of the on-shell $\mathcal{N}=(1,1)$ supersymmetry invariants of canonical dimensions 8 and 10 in $\mathcal{N}=(1,0)$ superspace. All possible $d=6, \mathcal{N}=(1,1)$ invariants 
were shown to vanish on shell, confirming the UV finiteness of $\mathcal{N}=(1,1) \mathrm{SYM}$ at one loop. We have also shown that there are no $d=8$ on-shell $\mathcal{N}=(1,1)$ supersymmetric invariants which possess the full off-shell $\mathcal{N}=(1,0)$ supersymmetry together with off-shell gauge invariance. Assuming the use of a gauge-invariant regularization scheme for $\mathcal{N}=(1,0)$ supergraphs, this implies the absence of two-loop counterterms. On the other hand, the on-shell $\mathcal{N}=(1,0)$ and $\mathcal{N}=(1,1)$ supersymmetric $d=8$ invariants exist. They are represented as the analytic harmonic $\mathcal{N}=(1,0)$ superspace integrals of densities which are both analytic and gauge-invariant only on mass shell, i.e. assuming the equations of motion to be satisfied. We show that one can enforce either off-shell analyticity or off-shell gauge invariance of the relevant density, but not both of them simultaneously. Structures of this kind appear in the derivative expansion of the supersymmetric Born-Infeld action.

Two $d=10$ invariants were explicitly constructed as integrals over the whole $\mathcal{N}=$ $(1,0)$ harmonic superspace. The single-trace invariant can be rewritten as an integral over the full $\mathcal{N}=(1,1)$ superspace, while the double-trace invariant cannot. This property, being combined with an additional reasoning based on the algebraic renormalization ideas adapted to the $\mathcal{N}=(1,0)$ harmonic superspace formalism, could potentially explain why the double-trace invariant is UV protected. However, to prove this, we would need first to compute the chain of $\mathcal{N}=(1,0)$ harmonic superspace co-forms associated to these two invariants and possibly establish that the chain associated to the double-trace invariant is, indeed, incompatible with the one of the classical action.

The $\mathcal{N}=(1,1)$ harmonic superspace is also useful to conveniently combine on-shell particle states into a G-analytic superfield in momentum space. This provides an efficient tool to apply the generalized unitary method to compute on-shell scattering amplitudes in $\mathcal{N}=(1,1), 6 D$ SYM theory [52] (see also [53] and references therein). It would be interesting to clarify the relations between the Feynman rules in $\mathcal{N}=(1,0)$ harmonic superspace and this generalized unitary method in momentum on-shell harmonic superspace.

We conclude by mentioning some other problems where our on-shell harmonic approach could be applied.

It could be used, e.g., to construct the invariants of higher dimension $d \geq 12$ in the $\mathcal{N}=(1,1), 6 D$ SYM theory and to inspect whether some kind of the non-renormalization theorems could be formulated there. The same methods could be applied for constructing the Born-Infeld effective action for coincident D5-branes in type IIB string theory, with the manifest $\mathcal{N}=(1,0)$ off-shell and hidden $\mathcal{N}=(0,1)$ on-shell supersymmetries. It would be also interesting to develop an analogous on-shell $\mathcal{N}=4,4 D$ harmonic superspace approach for the $\mathcal{N}=4,4 D$ SYM theory in the off-shell $\mathcal{N}=2$ superfield formulation and apply it to the problem of constructing the relevant quantum effective action and proving its identity with the appropriate D3-brane Born-Infeld action. An intriguing feature of such effective actions is the presence of Chern-Simons (or Wess-Zumino) type terms of non-tensorial character [54-56]. It would be interesting to see how such terms (and their possible 6D counterparts) could be identified in the on-shell harmonic superspace approach.

The last (but not least) domain where the on-shell harmonic superspace methods could help in selecting relevant counterterms and other higher-dimensional invariants is extended supergravities in diverse dimensions. 


\section{Acknowledgments}

E.I. acknowledges the support from the RFBR grant 15-02-06670 and a grant of the IN2P3JINR Program. E.I. and G.B would like to thank SUBATECH, Université de Nantes, and A.S. would like to thank Ecole Polytechnique and JINR for the warm hospitality in the course of this study. The authors thank Joseph Buchbinder, Shahin Sheikh Jabbari, Emery Sokatchev and Kelly Stelle for the interest in the work and useful comments.

\section{A Bianchi identities}

Many important identities between the harmonic superfields in the pure gauge $\mathcal{N}=(1,0)$ theory were derived and discussed in section 3. The Bianchi relations allow one to obtain further interesting and useful identities.

When one includes the hypermultiplet superfields and imposes the on-shell constraints (4.8), (4.9), many other relations relevant to $\mathcal{N}=(1,1)$ SYM theory can also be derived.

\section{A.1 Off-shell relations}

We first discuss the pure gauge theory off-shell relations. Taking the anticommutator of $D_{b}^{+}$with the second of relation in (3.34), we find

$$
\left[\nabla_{a b}, \nabla_{c d}\right]=\frac{1}{4}\left(\varepsilon_{a c d f} \nabla_{b}^{-} W^{+f}+\varepsilon_{b c d f} D_{a}^{+} W^{-f}\right) .
$$

An important Bianchi identity is obtained from (A.1) by contracting its both sides with $\varepsilon^{a b c d}$ and using the fact that $\left[\nabla_{a b}, \nabla_{c d}\right] \varepsilon^{a b c d} \equiv 0$. One obtains

$$
\nabla_{b}^{-} W^{+b}=D_{b}^{+} W^{-b} \text {. }
$$

Rewriting the identity (3.39) as

$$
D_{a}^{+} W^{+b}=\frac{1}{4} \delta_{a}^{b} D_{c}^{+} W^{+c},
$$

acting on its both sides by $\nabla^{--}$and using (A.2), one also finds

$$
D_{a}^{+} W^{-b}+\nabla_{a}^{-} W^{+b}=\frac{1}{2} \delta_{a}^{b} \nabla_{c}^{-} W^{+c}=\frac{1}{2} \delta_{a}^{b} D_{c}^{+} W^{-c} .
$$

One of the corollaries of (A.1) is that its right-hand side is antisymmetric under the permutations $a \leftrightarrow b$ and $a b \leftrightarrow c d$, as its left-hand side is (the antisymmetry under $c \leftrightarrow d$ is manifest on both sides).

One more important Bianchi identity following from the basic (anti)commutation relations can be derived by anticommuting both sides of (A.1) with $D_{a}^{+}$or $\nabla_{b}^{-}$,

$$
\nabla_{a b} W^{+b}=-\frac{i}{4} D_{a}^{+} \nabla_{b}^{-} W^{+b}=-\frac{i}{4} D_{a}^{+} D_{b}^{+} W^{-b} \Leftrightarrow D_{a}^{+} \nabla_{b}^{-} W^{+b}+\frac{1}{2} \nabla_{a}^{-} D_{b}^{+} W^{+b}=0 .
$$

All other relations obtained in this way are identically satisfied as a consequence of (A.5) and the identities derived above. By commuting (A.5) with $\nabla^{--}$, one obtains an analogous identity for $W^{-a}$,

$$
\nabla_{a b} W^{-b}=\frac{i}{4} \nabla_{a}^{-} \nabla_{b}^{-} W^{+b}=\frac{i}{4} \nabla_{a}^{-} D_{b}^{+} W^{-b} \Leftrightarrow \nabla_{a}^{-} D_{b}^{+} W^{-b}+\frac{1}{2} D_{a}^{+} \nabla_{b}^{-} W^{-b}=0 .
$$




\section{A.2 On-shell $\mathcal{N}=(1,1)$ relations}

The presence of the hypermultiplet does not affect the off-shell identities derived above. But the on-shell identities are modified. For example, instead of $D_{b}^{+} W^{+a}=0$, we obtain, using (3.39),

$$
D_{b}^{+} W^{+a}=-\frac{1}{2} \delta_{b}^{a}\left[q^{+A}, q_{A}^{+}\right]
$$

Bearing in mind (A.7), one obtains

$$
\begin{aligned}
D_{a}^{+} W^{-a} & =\nabla_{a}^{-} W^{+a}=-2\left[q^{-A}, q_{A}^{+}\right], \quad \nabla_{a}^{-} W^{-a}=-2\left[q^{-A}, q_{A}^{-}\right], \\
\nabla_{a b} W^{+b} & =-\frac{i}{2}\left[\nabla_{a}^{-} q^{+C}, q_{C}^{+}\right]=\frac{i}{2}\left[D_{a}^{+} q^{-C}, q_{C}^{+}\right], \\
\nabla_{a b} W^{-b} & =\frac{i}{2}\left[D_{a}^{+} q^{-C}, q_{C}^{-}\right]=-\frac{i}{2}\left[\nabla_{a}^{-} q^{+C}, q_{C}^{-}\right] .
\end{aligned}
$$

Starting from the evident identity

$$
\nabla_{a b} D_{c}^{+} q^{-A}=\frac{1}{2 i}\left\{D_{a}^{+}, \nabla_{b}^{-}\right\} D_{c}^{+} q^{-A},
$$

and repeatedly using (3.34) together with the on-shell relations (4.10), it is straightforward to obtain the following cyclic on-shell identity,

$$
\left(\nabla_{a b} D_{c}^{+}+\nabla_{c a} D_{b}^{+}+\nabla_{b c} D_{a}^{+}\right) q^{-A}=\frac{i}{2} \varepsilon_{a b c d}\left(\left[W^{-d}, q^{+A}\right]-\left[W^{+d}, q^{-A}\right]\right),
$$

which, in virtue of the analyticity condition $D_{a}^{+} q^{+A}=0$, also implies

$$
\left(\nabla_{a b} \nabla_{c}^{-}+\nabla_{c a} \nabla_{b}^{-}+\nabla_{b c} \nabla_{a}^{-}\right) q^{+A}=-\frac{i}{2} \varepsilon_{a b c d}\left(\left[W^{-d}, q^{+A}\right]-\left[W^{+d}, q^{-A}\right]\right) .
$$

Some useful consequences of these identities are

$$
\begin{aligned}
\nabla^{a b} D_{b}^{+} q_{A}^{-} & =\frac{i}{2}\left(\left[W^{+a}, q_{A}^{-}\right]-\left[W^{-a}, q_{A}^{+}\right]\right), \\
D_{b}^{+} \nabla^{a b} q_{A}^{-} & =-\frac{i}{2}\left(\left[W^{-a}, q_{A}^{+}\right]+2\left[W^{+a}, q_{A}^{-}\right]\right), \\
\nabla^{a d} \nabla_{d e} q_{A}^{+} & =-\frac{1}{4}\left[D_{e}^{+} W^{-a}, q_{A}^{+}\right]-\frac{1}{4} \delta_{e}^{a}\left(\left\{W^{+f}, D_{f}^{+} q_{A}^{-}\right\}-\frac{1}{2}\left[q_{A}^{-},\left[q^{+C}, q_{C}^{+}\right]\right]\right), \\
\nabla^{a d} \nabla_{d e} q_{A}^{-} & =\frac{1}{4}\left[\nabla_{e}^{-} W^{+a}, q_{A}^{-}\right]-\frac{1}{4} \delta_{e}^{a}\left(\left\{W^{-f}, D_{f}^{+} q_{A}^{-}\right\}+\frac{1}{2}\left[q_{A}^{+},\left[q^{-C}, q_{C}^{-}\right]\right]\right), \\
\nabla^{c d} \nabla_{c d} q_{A}^{-} & =\left\{W^{-e}, D_{e}^{+} q_{A}^{-}\right\}+\frac{1}{2}\left[q_{A}^{+},\left[q^{-C}, q_{C}^{-}\right]\right]-\frac{1}{2}\left[q_{A}^{-},\left[q^{-C}, q_{C}^{+}\right]\right], \\
\nabla^{c d} \nabla_{c d} q_{A}^{+} & =\left\{W^{+e}, D_{e}^{+} q_{A}^{-}\right\}+\frac{1}{2}\left[q_{A}^{+},\left[q^{-C}, q_{C}^{+}\right]\right]-\frac{1}{2}\left[q_{A}^{-},\left[q^{+C}, q_{C}^{+}\right]\right], \\
D_{a}^{+} D_{b}^{+} \nabla^{a b} q_{A}^{-} & =i\left(\left\{W^{+e}, D_{e}^{+} q_{A}^{-}\right\}-\left[q_{A}^{+},\left[q^{-C}, q_{C}^{+}\right]\right]-2\left[q_{A}^{-},\left[q^{+C}, q_{C}^{+}\right]\right]\right), \\
D_{a}^{+} \nabla^{a d} D_{d}^{+} q_{A}^{-} & =-\frac{i}{2}\left(\left\{W^{+e}, D_{e}^{+} q_{A}^{-}\right\}+2\left[q_{A}^{+},\left[q^{-C}, q_{C}^{+}\right]\right]-2\left[q_{A}^{-},\left[q^{+C}, q_{C}^{+}\right]\right]\right),
\end{aligned}
$$

where we took advantage of the on-shell relations (A.8). Note that (A.12), (A.16) and (A.17) are none other than the covariant superfield form of the $6 D$ Dirac and KleinFock-Gordon equations for the physical fermionic and bosonic components of $q_{A}^{+}$. 
One more useful on-shell consequence of the Bianchi identities is the following cyclic identity:

$$
\nabla^{a b} W^{+c}+\nabla^{c a} W^{+b}+\nabla^{b c} W^{+a}=\frac{i}{2} \varepsilon^{a b c d} \nabla_{d}^{-}\left[q^{+A}, q_{A}^{+}\right]
$$

from which one can derive

$$
\nabla^{b c} \nabla_{b c} W^{+a}=\left[\nabla_{d}^{-} W^{+a}, W^{+d}\right]+2 i \nabla^{a b} \nabla_{b}^{-}\left(q^{+}\right)^{2}+\frac{1}{2}\left[\left[q^{-A}, q_{A}^{+}\right], W^{+a}\right] .
$$

\section{B In quest of an off-shell $\mathcal{N}=(1,1) d=6$ invariant}

We continue here the discussion of subsection 5.1 and study the symmetries of the higher derivative actions of canonical dimension 6 . A generic $\mathcal{N}=(1,0)$ supersymmetric action of this kind is a linear combination of the supergauge action (5.1) and the hypermultiplet actions (4.11), (5.3) and (5.5). The question is whether one can define a specific linear combination $S^{(6)}$ which would be invariant off shell under the variations (4.15) of the hidden $\mathcal{N}=(0,1)$ supersymmetry.

Requiring the cancellation of the terms generated by the variations (4.15) in the first order in $q^{+A}$ and using the formula

$$
\delta S_{\mathrm{SYM}}^{(6)}=\frac{1}{2 g^{2}} \operatorname{Tr} \int d Z \delta V^{++}\left(\nabla^{--}\right)^{2} F^{++}=\frac{1}{2 g^{2}} \operatorname{Tr} \int d \zeta^{(-4)} \delta V^{++}\left(D^{+}\right)^{4}\left(\nabla^{--}\right)^{2}\left(D^{+}\right)^{4} V^{--},
$$

we deduce the following form for the candidate action

$$
\begin{aligned}
S^{(6)}= & \frac{1}{2 g^{2}} \operatorname{Tr}\left[\int d \zeta^{(-4)}\left(F^{++}\right)^{2}-\int d Z q^{+A} \nabla^{--} q_{A}^{+}-\frac{1}{2} \int d Z q^{+A}\left(\nabla^{--}\right)^{2} \nabla^{++} q_{A}^{+}\right] \\
& +\sum_{n \geq 3} \alpha_{n} S_{n}+\beta S_{\text {quart }},
\end{aligned}
$$

where $\alpha_{n}$ and $\beta$ are arbitrary coefficients; $S_{n}$ and $S_{\text {quart }}$ were defined in (5.3), (5.5). The variation of the second line in (B.2) does not involve the linear in $q$ terms (this is obvious for $S_{\text {quart }}$ and one can also show this to be true for all $S_{n}$ with $n \geq 3$ ). These terms are also canceled in the variation of the first line.

The variation of the first line in (B.2) can be represented as

$$
\begin{aligned}
\delta S_{\text {first line }}^{(6)}= & \frac{1}{4 g^{2}} \operatorname{Tr} \int d \zeta^{(-4)}\left[\left(D^{+}\right)^{4} C^{-3 A}\right] \nabla^{++} q_{A}^{+} \\
& -\frac{1}{2 g^{2}} \operatorname{Tr} \int d \zeta^{(-4)}\left[\left(D^{+}\right)^{4}\left(\epsilon^{-A} V^{--}, q_{A}^{+}\right]\left[q_{B}^{+}, q^{+B}\right],\right.
\end{aligned}
$$

where

$$
\begin{aligned}
C^{-3 A}= & \epsilon^{-B}\left[q_{B}^{+},\left(\nabla^{--}\right)^{2} q^{+A}\right]+\epsilon^{-A}\left[\nabla^{--} q_{B}^{+}, \nabla^{--} q^{+B}\right] \\
& +\left[\nabla^{--} \delta_{0} V^{--}, q^{+A}\right]-2\left[\delta_{0} V^{--}, \nabla^{--} q^{+A}\right]
\end{aligned}
$$

or, in the equivalent form,

$$
\begin{aligned}
C^{-3 A}= & \epsilon^{-B}\left[q_{B}^{+},\left(\nabla^{--}\right)^{2} q^{+A}\right]-\epsilon^{+B}\left[\left(\nabla^{--}\right)^{2} q_{B}^{+}, \nabla^{--} q^{+A}\right] \\
& +\left[\nabla^{--} \delta_{0} V^{--}, q^{+A}\right]+\left[\nabla^{++}\left(\nabla^{--} \delta_{0} V^{--}\right), \nabla^{--} q^{+A}\right] .
\end{aligned}
$$

While calculating this variation, we took advantage of the general formula (3.28) for the variation of $V^{--}$. 
The second term in the variation (B.3) cancels the variation of the quartic term $S_{\text {quart }}$ in $S^{(6)}$ under a particular choice $\beta=\frac{1}{8 g^{2}}$ that we adopt. Next, we note that the second term in (B.2) can be rewritten as an integral over the analytic subspace,

$$
\operatorname{Tr} \int d Z q^{+A} \nabla^{--} q_{A}^{+}=-\operatorname{Tr} \int d \zeta^{(-4)} F^{++}\left[q^{+A}, q_{A}^{+}\right] .
$$

Then the first two terms in (B.2) together with the quartic term can be written as

$$
\frac{1}{2 g^{2}} \operatorname{Tr} \int d \zeta^{(-4)}\left(F^{++}+\frac{1}{2}\left[q^{+A}, q_{A}^{+}\right]\right)^{2} .
$$

Note that the expression in the parentheses is nothing but the equation of motion for $V^{++}$ for the standard $d=4$ action $S^{V q^{+}}$of eq. (4.14). Then the action $S^{(6)}$ can be written as

$$
S^{(6)}=\frac{1}{2 g^{2}} \operatorname{Tr}\left[\int d \zeta^{(-4)}\left(F^{++}+\frac{1}{2}\left[q^{+A}, q_{A}^{+}\right]\right)^{2}-\frac{1}{2} \int d Z\left(\nabla^{--}\right)^{2} q^{+A} \nabla^{++} q_{A}^{+}\right] .
$$

Similar to the first term in (B.7), the second term is also the product of two equivalent forms of the equation of motion for $q^{+A}$, eqs. (4.8) and (4.9).

The total variation of (B.7) is given by the first term in the variation (B.3). It does not seem to represent a total derivative. If adding the sum over $S_{n}$ with nonzero coefficients, the cubic in $q$ terms in the variation are modified, and they would include the terms with higher powers of harmonic derivatives. This does not seem to help.

Let us focus on the case of vanishing $\alpha_{n}$. To check that the integrand in the variation of such an action is not a total derivative and hence that (B.3) does not vanish, we can use the following trick. If the integrand were a total derivative, the related "equation of motion" obtained by varying (B.3) with respect to $q^{+A}$, treated as an unconstrained superfield on the analytic harmonic superspace, should identically vanish. After some algebra, with making use of the analyticity of $q^{+A}$, we find that the variational derivative of (B.3) with respect to $q^{+A}$ is reduced to the expression

$$
\frac{\delta\left(\delta_{0} S_{2}^{\prime}\right)}{\delta q_{A}^{+}} \sim\left(D^{+}\right)^{4} Y^{-A}
$$

where

$$
\begin{aligned}
Y^{-A}= & \epsilon^{+A}\left[q^{+D},\left(\nabla^{--}\right)^{2} q_{D}^{+}\right]+2\left[\left(\nabla^{--} \delta_{0} V^{--}\right), \nabla^{++} q^{+A}\right] \\
& +2\left[\delta_{0} V^{--},\left(1+\left\{\nabla^{++}, \nabla^{--}\right\}\right) q^{+A}\right] \\
& -\frac{\delta\left(\delta_{0} V^{--}\right)}{\delta q_{A}^{+}}\left(\left[q^{+B}, \nabla^{--} \nabla^{++} q_{B}^{+}\right]+3\left[\nabla^{--} q^{+B}, \nabla^{++} q_{B}^{+}\right]\right) .
\end{aligned}
$$

It is easy to show that for the particular class of $q^{+A}$ subjected to the dynamical equations (4.8) and (4.9), the variation $\delta_{0} V^{--}$is reduced to

$$
\delta_{0} V^{--} \mid=\epsilon^{-A} \nabla^{--} q_{A}^{+},
$$

and

$$
Y^{A} \mid=4 \epsilon^{-B}\left[\nabla^{--} q_{B}^{+}, q^{+A}\right] .
$$

Then it immediately follows that $\left(D^{+}\right)^{4}\left(Y^{A} \mid\right)=0$ because of the analyticity of $q^{+A}$. 
However, we do not see any reason for (B.8) to vanish in the general case, when $q^{+A}$ is not subject to any dynamical constraint. One of the arguments against the existence of an off-shell $\mathcal{N}=(1,1)$ supersymmetric $d=6$ action is the following. In the $d=$ 4 action (4.14), the extended supersymmetry implies among other things the symmetry between the physical fermion of the gauge multiplet and the fermion of the hypermultiplet. This symmetry is manifest when the action is expressed in components. On the other hand, there is no such symmetry for the $d=6$ action. It was shown in [41] that an action $S_{n}$ in (5.3) involves an infinite number of physical propagating bosons and fermions associated with the harmonic expansion of $q^{+A}$. This cannot match with the gluino sector that involves a finite number $(=12)$ of the fermionic degrees of freedom for each value of the color index.

\section{C $\mathcal{N}=(1,0)$ on-shell $d=8$ invariants}

We give here the full list of the planar (single-trace) G-analytic $d=8$ invariants $^{18}$ involving the gauge supermultiplet and the hypermultiplet and study their properties.

\section{C.1 Most general analytic $\mathcal{L}^{+4}$}

A generic on-shell analytic $d=8$ single-trace Lagrangian $\mathcal{L}^{+4}$ can be represented as a linear combination of the following six terms,

$$
\mathcal{L}_{\text {gen }}^{+4}=L_{0}^{+4}+\sum_{i=1}^{5} \alpha_{i} L_{i}^{+4},
$$

where $L_{0}^{+4}$ is given by $(5.25)$ and

$$
\begin{aligned}
L_{1}^{+4}= & \operatorname{Tr}\left\{i\left[q^{+A}, \nabla_{a b} q_{A}^{+}\right] W^{+a} W^{+b}+\frac{1}{2}\left\{W^{+a},\left(q^{+}\right)^{2}\right\}\left[D_{a}^{+} q^{-A}, q_{A}^{+}\right]+\left(q^{+}\right)^{4}\left[q^{-A}, q_{A}^{+}\right]\right\}, \\
L_{2}^{+4}= & \operatorname{Tr}\left\{q^{+A} \nabla_{a b} q_{A}^{+} q^{+B} \nabla^{a b} q_{B}^{+}+q^{+A} D_{a}^{+} q_{A}^{-}\left[q^{+B} W^{+a} q_{B}^{+}-\left(q^{+}\right)^{2} W^{+a}\right]\right. \\
& \left.+\left(q^{+}\right)^{2} q^{+B} q^{+A} q_{A}^{-} q_{B}^{+}+\left(q^{+}\right)^{4} q^{+A} q_{A}^{-}\right\}, \\
L_{3}^{+4}= & \operatorname{Tr}\left\{q^{+A} \nabla_{a b} q_{A}^{+} \nabla^{a b} q^{+B} q_{B}^{+}-\frac{1}{2} q^{+A} D_{a}^{+} q_{A}^{-}\left[q^{+B} W^{+a} q_{B}^{+}-W^{+a}\left(q^{+}\right)^{2}\right]\right. \\
& +\frac{1}{2} D_{a}^{+} q^{-A} q_{A}^{+}\left[q^{+B} W^{+a} q_{B}^{+}-\left(q^{+}\right)^{2} W^{+a}\right]+\frac{1}{2}\left(q^{+}\right)^{4}\left(q^{-A} q_{A}^{+}-q^{+A} q_{A}^{-}\right) \\
& \left.+\frac{1}{2}\left(q^{+}\right)^{2} q^{+A}\left(q^{-B} q_{B}^{+}-q^{+B} q_{B}^{-}\right) q_{A}^{+}\right\}, \\
L_{4}^{+4}= & \operatorname{Tr}\left\{\nabla_{a b} q^{+B} q_{B}^{+} \nabla^{a b} q^{+A} q_{A}^{+}+D_{a}^{+} q^{-A} q_{A}^{+}\left[W^{+a}\left(q^{+}\right)^{2}-q^{+B} W^{+a} q_{B}^{+}\right]\right. \\
& \left.-\left(q^{+}\right)^{2}\left[q^{+A} q^{-B} q_{B}^{+} q_{A}^{+}+\left(q^{+}\right)^{2} q^{-B} q_{B}^{+}\right]\right\}, \\
L_{5}^{+4}= & \operatorname{Tr}\left\{\nabla_{a b} q^{+B} \nabla^{a b} q_{B}^{+}\left(q^{+}\right)^{2}+\frac{1}{2}\left[q^{+A}, W^{+a}\right]\left[D_{a}^{+} q_{A}^{-},\left(q^{+}\right)^{2}\right]\right. \\
& \left.+\frac{1}{2}\left[q^{+A},\left(q^{+}\right)^{2}\right]\left[q_{A}^{-},\left(q^{+}\right)^{2}\right]\right\} .
\end{aligned}
$$

\footnotetext{
${ }^{18}$ The pure $\mathcal{N}=(1,0)$ SYM invariant (5.21) is G-analytic, and its $\mathcal{N}=(1,1)$ extension we are interested in is also G-analytic.
} 
Here $\left(q^{+}\right)^{2}:=q^{+A} q_{A}^{+}=\frac{1}{2}\left[q^{+A}, q_{A}^{+}\right]$. It is straightforward to check that each term in the sum (C.1) is separately annihilated by $D_{f}^{+}$.

Taking into account that the analytic superspace integration measure is $\sim d^{6} x d u\left(D_{a}^{-}\right)^{4}$, one can integrate the above Lagrangians by parts not only with respect to $\nabla_{a b}$ but also with respect to $\nabla_{a}^{-}$and $\nabla^{--}$. Using this opportunity and making use of the on-shell relation (A.17), one can show that $L_{1}^{+4}$ is a total derivative on shell and establish the following on-shell relations,

$$
L_{2}^{+4}=L_{4}^{+4}, \quad L_{5}^{+4}=-L_{2}^{+4}-L_{3}^{+4}
$$

We are left with only two independent Lagrangians, $L_{2}^{+4}$ and $L_{3}^{+4}$. The representation (C.1) is thus rewritten, modulo a total derivative, as

$$
\mathcal{L}_{\text {gen }}^{+4}=L_{0}^{+4}+\alpha_{2} L_{2}^{+4}+\alpha_{3} L_{3}^{+4}
$$

\section{C.2 Hidden $\mathcal{N}=(0,1)$ supersymmetry}

Now we can vary $\mathcal{L}_{\text {gen }}^{+4}$ with respect to the on-shell $\mathcal{N}=(0,1)$ transformations $(4.28)-(4.31)$ in order to learn at which values of the free parameters $\alpha_{i}$ it is invariant (up to a total derivative). A general variation contains the terms not involving the superfield strengths $W^{ \pm a}$ as well as the terms of the first, second and third degrees in $W^{ \pm a}$. It is easy to check that the cubic term $\sim(W)^{3}$ in the variation comes only from $L_{0}^{+4}$ and represents a total derivative. To explore the cancellation of the other terms [i.e. terms $\sim(W)^{2},(W)^{1}$ and $\left.(W)^{0}\right]$ in the variation of the generic Lagrangian (C.8) is not an easy problem.

Consider a symmetrized trace invariant (5.34), which, as we showed, is the on-shell $\mathcal{N}=(1,1)$ invariant. It can be expressed via the structures $(5.25),($ C.2) $-($ C.6) as

$$
\mathcal{L}_{(1,1)}^{+4}=L_{0}^{+4}+L_{1}^{+4}-\frac{1}{6}\left(L_{2}^{+4}+L_{4}^{+4}-4 L_{3}^{+4}+2 L_{5}^{+4}\right)
$$

This corresponds to the following particular choice of the coefficients in the general formula (C.1),

$$
\alpha_{1}=1, \quad \alpha_{2}=\alpha_{4}=-\frac{1}{6}, \quad \alpha_{3}=\frac{2}{3}, \quad \alpha_{5}=-\frac{1}{3} .
$$

Taking into account the on-shell equivalence relations (C.7), the invariant (C.9) can be cast, up to a total derivative and modulo equations of motion, in the simple form (C.8) with $\alpha_{2}=0, \alpha_{3}=1$ :

$$
\mathcal{L}_{(1,1)}^{+4}=L_{0}^{+4}+L_{3}^{+4}
$$

We have explicitly checked the cancellation, up to a total derivative, of the quadratic terms $\sim(W)^{2}$ in the variation of this expression under (4.28)-(4.31).

Open Access. This article is distributed under the terms of the Creative Commons Attribution License (CC-BY 4.0), which permits any use, distribution and reproduction in any medium, provided the original author(s) and source are credited. 


\section{References}

[1] P.H. Frampton and T.W. Kephart, Explicit Evaluation of Anomalies in Higher Dimensions, Phys. Rev. Lett. 50 (1983) 1343 [Erratum ibid. 51 (1983) 232] [INSPIRE].

[2] L. Álvarez-Gaumé and E. Witten, Gravitational Anomalies, Nucl. Phys. B 234 (1984) 269 [INSPIRE].

[3] B. Zumino, Y.-S. Wu and A. Zee, Chiral Anomalies, Higher Dimensions and Differential Geometry, Nucl. Phys. B 239 (1984) 477 [inSPIRE].

[4] A.V. Smilga, Chiral anomalies in higher-derivative supersymmetric $6 D$ theories, Phys. Lett. B 647 (2007) 298 [hep-th/0606139] [INSPIRE].

[5] A.A. Tseytlin, On nonAbelian generalization of Born-Infeld action in string theory, Nucl. Phys. B 501 (1997) 41 [hep-th/9701125] [INSPIRE].

[6] P. Koerber and A. Sevrin, The NonAbelian Born-Infeld action through order $\alpha^{\prime 3}$, JHEP 10 (2001) 003 [hep-th/0108169] [INSPIRE].

[7] L. De Fosse, P. Koerber and A. Sevrin, The Uniqueness of the Abelian Born-Infeld action, Nucl. Phys. B 603 (2001) 413 [hep-th/0103015] [InSPIRE].

[8] P. Koerber and A. Sevrin, The NonAbelian D-brane effective action through order $\alpha^{\prime 4}$, JHEP 10 (2002) 046 [hep-th/0208044] [INSPIRE].

[9] J.M. Drummond, P.J. Heslop, P.S. Howe and S.F. Kerstan, Integral invariants in $N=4$ SYM and the effective action for coincident D-branes, JHEP 08 (2003) 016 [hep-th/0305202] [INSPIRE].

[10] P.S. Howe and K.S. Stelle, Ultraviolet Divergences in Higher Dimensional Supersymmetric Yang-Mills Theories, Phys. Lett. B 137 (1984) 175 [INSPIRE].

[11] G. Bossard, P.S. Howe and K.S. Stelle, The Ultra-violet question in maximally supersymmetric field theories, Gen. Rel. Grav. 41 (2009) 919 [arXiv:0901.4661] [InSPIRE].

[12] A. Galperin, E. Ivanov, S. Kalitzin, V. Ogievetsky and E. Sokatchev, Unconstrained $N=2$ Matter, Yang-Mills and Supergravity Theories in Harmonic Superspace, Class. Quant. Grav. 1 (1984) 469 [Erratum ibid. 2 (1985) 127] [INSPIRE].

[13] A.S. Galperin, E.A. Ivanov, V.I. Ogievetsky and E.S. Sokatchev, Harmonic Superspace, Cambridge University Press, (2001), pg. 306.

[14] P.S. Howe, K.S. Stelle and P.C. West, $\mathcal{N}=1 D=6$ harmonic superspace, Class. Quant. Grav. 2 (1985) 815 [InSPIRE].

[15] B.M. Zupnik, Six-dimensional Supergauge Theories in the Harmonic Superspace, Sov. J. Nucl. Phys. 44 (1986) 512 [inSPIRE].

[16] B.M. Zupnik, The Action of the Supersymmetric $\mathcal{N}=2$ Gauge Theory in Harmonic Superspace, Phys. Lett. B 183 (1987) 175 [inSPIRE].

[17] Z. Bern, L.J. Dixon and V.A. Smirnov, Iteration of planar amplitudes in maximally supersymmetric Yang-Mills theory at three loops and beyond, Phys. Rev. D 72 (2005) 085001 [hep-th/0505205] [INSPIRE].

[18] Z. Bern, J.J.M. Carrasco, L.J. Dixon, H. Johansson and R. Roiban, The Complete Four-Loop Four-Point Amplitude in $N=4$ super-Yang-Mills Theory, Phys. Rev. D 82 (2010) 125040 [arXiv: 1008.3327] [INSPIRE]. 
[19] Z. Bern, J.J.M. Carrasco, L.J. Dixon, H. Johansson and R. Roiban, Simplifying Multiloop Integrands and Ultraviolet Divergences of Gauge Theory and Gravity Amplitudes, Phys. Rev. D 85 (2012) 105014 [arXiv:1201.5366] [INSPIRE].

[20] O. Piguet and S.P. Sorella, Algebraic renormalization: Perturbative renormalization, symmetries and anomalies, Lect. Notes Phys. M 28 (1995) 1 [INSPIRE].

[21] G. Bossard, P.S. Howe, U. Lindström, K.S. Stelle and L. Wulff, Integral invariants in maximally supersymmetric Yang-Mills theories, JHEP 05 (2011) 021 [arXiv:1012.3142] [INSPIRE].

[22] N. Berkovits, M.B. Green, J.G. Russo and P. Vanhove, Non-renormalization conditions for four-gluon scattering in supersymmetric string and field theory, JHEP 11 (2009) 063 [arXiv: 0908.1923] [INSPIRE].

[23] J. Bjornsson and M.B. Green, 5 loops in 24/5 dimensions, JHEP 08 (2010) 132 [arXiv: 1004.2692] [INSPIRE].

[24] J. Bjornsson, Multi-loop amplitudes in maximally supersymmetric pure spinor field theory, JHEP 01 (2011) 002 [arXiv:1009.5906] [INSPIRE].

[25] Z. Bern, S. Davies, T. Dennen and Y.-t. Huang, Absence of Three-Loop Four-Point Divergences in $\mathcal{N}=4$ Supergravity, Phys. Rev. Lett. 108 (2012) 201301 [arXiv:1202.3423] [INSPIRE].

[26] P. Tourkine and P. Vanhove, An $R^{4}$ non-renormalisation theorem in $N=4$ supergravity, Class. Quant. Grav. 29 (2012) 115006 [arXiv:1202.3692] [INSPIRE].

[27] Z. Bern, S. Davies, T. Dennen and Y.-t. Huang, Ultraviolet Cancellations in Half-Maximal Supergravity as a Consequence of the Double-Copy Structure, Phys. Rev. D 86 (2012) 105014 [arXiv: 1209.2472] [INSPIRE].

[28] Z. Bern, S. Davies and T. Dennen, Enhanced ultraviolet cancellations in $\mathcal{N}=5$ supergravity at four loops, Phys. Rev. D 90 (2014) 105011 [arXiv: 1409.3089] [InSPIRE].

[29] S. Deser, J.H. Kay and K.S. Stelle, Renormalizability Properties of Supergravity, Phys. Rev. Lett. 38 (1977) 527 [arXiv:1506.03757] [INSPIRE].

[30] S. Deser and J.H. Kay, Three Loop Counterterms for Extended Supergravity, Phys. Lett. B 76 (1978) 400 [INSPIRE].

[31] G. Bossard, C. Hillmann and H. Nicolai, $E_{7(7)}$ symmetry in perturbatively quantised $\mathcal{N}=8$ supergravity, JHEP 12 (2010) 052 [arXiv: 1007.5472] [INSPIRE].

[32] G. Bossard, P.S. Howe and K.S. Stelle, On duality symmetries of supergravity invariants, JHEP 01 (2011) 020 [arXiv: 1009.0743] [INSPIRE].

[33] N. Beisert, H. Elvang, D.Z. Freedman, M. Kiermaier, A. Morales and S. Stieberger, $E_{7(7)}$ constraints on counterterms in $\mathcal{N}=8$ supergravity, Phys. Lett. B 694 (2011) 265 [arXiv: 1009.1643] [INSPIRE].

[34] G. Bossard, P.S. Howe, K.S. Stelle and P. Vanhove, The vanishing volume of $D=4$ superspace, Class. Quant. Grav. 28 (2011) 215005 [arXiv:1105.6087] [INSPIRE].

[35] Z. Bern, J.J. Carrasco, L.J. Dixon, H. Johansson, D.A. Kosower and R. Roiban, Three-Loop Superfiniteness of $N=8$ Supergravity, Phys. Rev. Lett. 98 (2007) 161303 [hep-th/0702112] [INSPIRE]. 
[36] Z. Bern, J.J.M. Carrasco, L.J. Dixon, H. Johansson and R. Roiban, Manifest Ultraviolet Behavior for the Three-Loop Four-Point Amplitude of $\mathcal{N}=8$ Supergravity, Phys. Rev. D 78 (2008) 105019 [arXiv:0808.4112] [INSPIRE].

[37] Z. Bern, J.J. Carrasco, L.J. Dixon, H. Johansson and R. Roiban, The Ultraviolet Behavior of $\mathcal{N}=8$ Supergravity at Four Loops, Phys. Rev. Lett. 103 (2009) 081301 [arXiv:0905.2326] [INSPIRE].

[38] G. Bossard, P.S. Howe and K.S. Stelle, Invariants and divergences in half-maximal supergravity theories, JHEP 07 (2013) 117 [arXiv: 1304.7753] [INSPIRE].

[39] R.E. Kallosh, Counterterms in extended supergravities, Phys. Lett. B 99 (1981) 122 [INSPIRE].

[40] E.A. Ivanov, A.V. Smilga and B.M. Zupnik, Renormalizable supersymmetric gauge theory in six dimensions, Nucl. Phys. B 726 (2005) 131 [hep-th/0505082] [INSPIRE].

[41] E.A. Ivanov and A.V. Smilga, Conformal properties of hypermultiplet actions in six dimensions, Phys. Lett. B 637 (2006) 374 [hep-th/0510273] [INSPIRE].

[42] P.S. Howe, G. Sierra and P.K. Townsend, Supersymmetry in Six-Dimensions, Nucl. Phys. B 221 (1983) 331 [inSPIRE].

[43] E. Bergshoeff, M. Rakowski and E. Sezgin, Higher derivative super Yang-Mills theories, Phys. Lett. B 185 (1987) 371 [INSPIRE].

[44] E. Witten, Dynamical Breaking of Supersymmetry, Nucl. Phys. B 188 (1981) 513 [InSPIRE].

[45] D. Robert and A.V. Smilga, Supersymmetry vs ghosts, J. Math. Phys. 49 (2008) 042104 [math-ph/0611023] [INSPIRE].

[46] I.L. Buchbinder, A. Yu. Petrov and A.A. Tseytlin, Two loop $N=4$ super Yang-Mills effective action and interaction between D3-branes, Nucl. Phys. B 621 (2002) 179 [hep-th/0110173] [INSPIRE].

[47] I.L. Buchbinder and E.A. Ivanov, Complete $\mathcal{N}=4$ structure of low-energy effective action in $\mathcal{N}=4$ super Yang-Mills theories, Phys. Lett. B 524 (2002) 208 [hep-th/0111062] [INSPIRE].

[48] I.L. Buchbinder, E.A. Ivanov and A. Yu. Petrov, Complete low-energy effective action in $\mathcal{N}=4$ SYM: $A$ direct $\mathcal{N}=2$ supergraph calculation, Nucl. Phys. B 653 (2003) 64 [hep-th/0210241] [INSPIRE].

[49] I.L. Buchbinder and N.G. Pletnev, Leading low-energy effective action in the $6 D$ hypermultiplet theory on a vector/tensor background, Phys. Lett. B 744 (2015) 125 [arXiv: 1502.03257] [INSPIRE].

[50] D.J. Gross and E. Witten, Superstring Modifications of Einstein's Equations, Nucl. Phys. B 277 (1986) 1 [INSPIRE].

[51] I.L. Buchbinder and N.G. Pletnev, Construction of $6 D$ supersymmetric field models in $\mathcal{N}=(1,0)$ harmonic superspace, Nucl. Phys. B 892 (2015) 21 [arXiv:1411.1848] [InSPIRE].

[52] T. Dennen, Y.-t. Huang and W. Siegel, Supertwistor space for $6 D$ maximal super Yang-Mills, JHEP 04 (2010) 127 [arXiv:0910.2688] [INSPIRE].

[53] L.V. Bork, D.I. Kazakov, M.V. Kompaniets, D.M. Tolkachev and D.E. Vlasenko, Divergences in maximal supersymmetric Yang-Mills theories in diverse dimensions, JHEP 11 (2015) 059 [arXiv: 1508.05570] [INSPIRE]. 
[54] A.A. Tseytlin and K. Zarembo, Magnetic interactions of D-branes and Wess-Zumino terms in super Yang-Mills effective actions, Phys. Lett. B 474 (2000) 95 [hep-th/9911246] [INSPIRE].

[55] K.A. Intriligator, Anomaly matching and a Hopf-Wess-Zumino term in $6 d, \mathcal{N}=(2,0)$ field theories, Nucl. Phys. B 581 (2000) 257 [hep-th/0001205] [INSPIRE].

[56] P.C. Argyres, A.M. Awad, G.A. Braun and F.P. Esposito, Higher derivative terms in $\mathcal{N}=2$ supersymmetric effective actions, JHEP 07 (2003) 060 [hep-th/0306118] [INSPIRE]. 